\title{
Efficient Computation of the Special Cases of the Generalized Bessel Function Distributions
}

\author{
Ilir F. Progri \\ Giftet Inc., 5 Euclid Ave. \#3, Worcester, MA 01610, USA \\ ORCID: 0000-0001-5197-1278
}

Correspondence should be addressed to Ilir Progri; iprogri@giftet.com

Received December 23, 2018; Revised January 12, 2018-April 4, 2019, Accepted July 16, 2019; Published November 1, 2019.

Scientific Editor-in-Chief/Editor: Ilir F. Progri

Copyright (C) 2019 Giftet Inc. All rights reserved. This work may not be translated or copied in whole or in part without written permission to the publisher (Giftet Inc., 5 Euclid Ave. \#3, Worcester, MA 01610, USA), except for brief excerpts in connection with reviews or scholarly analysis. Use in connection with any form of information storage and retrieval, electronic adaptation, computer software or by similar or dissimilar methodology now known or hereafter developed is forbidden. The use of the publication of trade names, trademarks, service marks, or similar terms, even if they are not identified as such, is not to be taken as an expression of opinion as to whether or not they are subject to proprietary rights.

This paper discusses efficient and original computations of the special cases of the generalized Bessel function distributions of the second kind. The journal paper is a masterpiece in the field of numerical methods, numerical linear algebra, and computational mathematics. The entire work in this journal paper is original and never presented before in the literature. The main focus of this journal paper is to fill in the gap or to deal with the special cases that were not covered in the two premier publications of Progri 2016 and 2018. In this journal paper the closed form expression and the efficient computation of the probability density function (pdf) and the cumulative distribution function (cdf) for five special cases is provided. For each generalized Bessel distribution model the closed form expression of the cdfs is given by means of Legendre continued fraction or Taylor series expansion of the modified Bessel functions of the second kind, for both integer and real values of a parameter, which leads to incomplete gamma functions, hypergeometric series, the Kampé de Fériet functions (or double hypergeometric series), and in two special cases of the Srivastava's triple hypergeometric series. Efficient computations of the algorithms (which are implemented in MATLAB functions) are also presented in the paper. Numerical results are derived for each case to validate the theoretical models presented in the paper.

Index Terms - Bessel functions, modified Bessel functions, cumulative distribution function, Kampé de Fériet function, Srivastava's triple hypergeometric series, closed form expression, signal analysis, generalized functions, time-domain analysis, incomplete gamma functions, hypergeometric series. 


\section{Introduction}

This journal paper discusses several special cases in the original closed form expressions of the generalized Bessel function cumulative distribution functions (cdf) of the second kind.

Why are these special cases so important? These special cases are important because they cover cases that were not discussed in either Progri 2016 [1] and Progri 2018 [2]. There was a provision made in Progri 2016 [1] and an attempt to derive the main special case which is the one corresponding to the integer values of $p$. However, the derivations in Progri 2016 [1] were not complete. Hence, the main focus on this journal paper is to derive this closed form expression of the generalized modified Bessel function distribution of the second kind for integer values of $p$.

Why was I not successful in deriving the entire, closed form expression of the generalized modified Bessel function distribution of the second kind for integer values of $p$ in 2016 ?

There are two main reasons why I was not successful to derive the entire, closed form expression of the generalized modified Bessel function distribution of the second kind for integer values of $p$ in 2016: I had a limited capability of the computation library and I was not following the right computation (or numerical) methodology.

First, what do I mean by not having an in depth understanding of the computation capability? The computation of the closed form expression of the generalized modified Bessel function distribution of the second kind for integer values of $p$ requires the computation of the Kampé de Fériet ${ }^{\mathrm{i}}$ function (or double hypergeometric series [1]-[16]) and in some cases the of Srivastava's triple hypergeometric series $F^{(3)}[x, y, z]$ ii $[18]$-[22] in MATLAB. Back in 2016 I was barely understanding the Kampé de Fériet function (or double hypergeometric series [1]-[16]) and I made an unsuccessful attempt to derive an inefficient implementation of the Srivastava's triple hypergeometric series $F^{(3)}[x, y, z]$ [18]-[22] which luckily I did not publish. This limitation in the computational capability was filled in 2018 with the arrival of the Progri 2018 [2] where I developed a profoundly improved implementation in MATLAB of the Kampé de Fériet function (or double hypergeometric series [1]-[16]). Moreover, this limitation in the computational capability was entirely eliminated in 2019 as discussed in Appendices A through F in this paper.
Second, what is the right computational methodology? The computational methodology has to do with a sequence of steps, methods, algorithms, processes, etc. that are required to follow in order to produce the desired outcome in the computation of the closed form expression the generalized modified Bessel function distribution of the second kind for integer values of $p$. The main issue here is that this is not a trivial problem. Therefore, because this is not a trivial problem it requires a more profoundly sophisticated computational methodology. The organization of this journal paper is such that it enables the reader to understand the steps that are required to derive the closed form expression the generalized modified Bessel function distribution of the second kind for integer values of $p$.

In additional to the in depth original, novel, and exceptional organization of this journal paper I am also giving an in depth explanation of the insights that directed me to come up with such a wonderful in depth organization.

In 2016 I published an exceptional journal paper called “Exponential generalized Beta distribution" see (Progri 2016, [3]) which would not have been possible without me working out a series of details in another journal paper (Progri 2016, "Hypergeometric function partial derivatives" [4]). In (Progri 2016, "Hypergeometric function partial derivatives" [4]) I had to develop a series of techniques for me to succeed in the computation of the "Exponential generalized Beta distribution" see (Progri 2016, [3]). This is exactly what computation methodology is all about.

Therefore, by way of comparison and contrast, in order for me to develop this absolutely outstanding journal paper I had to work out a series of details in at least three other journal papers, Progri 2016, [1], [4] and Progri 2018, [2].

In Progri 2016, [1] I was able to develop the insight that there are things that I can do in 2016 and there are things that I need to do later on. In Progri 2018, [2] I was able to validate exactly that same computational mentality (for example the series expansion of incomplete gamma function in odd and even components (see Progri, 2018 [2], (19)) that I initially architected in Progri 2016, [1]. In Progri 2016, [4] I was able to produce a series of techniques that I can use successfully in the implementation of the series of psi $\psi(k+1)$ Progri (2016, [4] (127)) function not known by a lot of scholars. In this journal paper I was able to integrate the computation of $\psi(k+1)$ and $\psi(k+p+1)$ with the computation of the so called modified generalized Kampé de Fériet function because this was the ONLY way to achieve the highest possible performance in 
computational speed and accuracy.

The issue, however, is that the efficient computation of these special cases cdfs require the computation of the several Kampé de Fériet functions [2], [10]-[13] and in some cases of the Srivastava's triple hypergeometric series $F^{(3)}[x, y, z]$ [18]-[22] in MATLAB. The main issue is the following: Is there an algorithm that we can apply to successfully produce an efficient MATLAB function of the Srivastava's triple hypergeometric series $F^{(3)}[x, y, z] \quad[18]-[22]$ ? Is this algorithm also computationally stable?

Since, the generalized Bessel functions cdfs require the computation of the Srivastava's triple hypergeometric series $F^{(3)}[x, y, z][18]-[22]$ and the latter is not yet available in MATLAB 2018b [30], Giftet Inc. is also taking on the task to compute the Srivastava's triple hypergeometric series $F^{(3)}[x, y, z]$ [18]-[22] as part of the Indoor Geolocation Systems MATLAB Library and publish the results of this work in Dr. Progri pioneer publication [17].

The journal paper is also an extension of the discussion of "Efficient computation of generalized Bessel function distributions," in Progri 2018, [2]. Therefore, things that already mentioned in Progri 2018, [2] (the efficient computation of the Kampé de Fériet function (or double hypergeometric series [1]-[16]), I am not going to reiterate here. In this journal paper I developed another option for the computation of the Kampé de Fériet function (or double hypergeometric series [1]-[16]) called option 2. Option 2 is the recursive implementation of Option 1. The main purpose of Option 2 is to eliminate division or multiplication of very large numbers. Since, in Option 1 the number of terms included in the computation of the Kampé de Fériet function is limited to about fifty; in Option 2 there is no limit on the number of terms included in the computation.

In this journal paper Dr. Progri masterfully, discusses the advantages and disadvantages of the direct closed form expressions vs. recursive closed form expressions.

Because of the level of detail, sophistication, brilliance in treating all these special cases, this paper presents an original work that falls into the category of masterpiece marvels in analytical derivations and computational mathematics series never published anywhere else.

This paper is organized as follows: in Sect. 2 special cases of the generalized Bessel function distribution of the second kind pdfs are discussed. The efficient computation of special cases of the generalized Bessel distribution of the second kind cdfs is presented in Sect. 3. Section 4 contains numerical, theoretical results; Conclusion is provided in Sect. 5 along with a list of references. An inefficient implementation of $\mathcal{F}_{21}(x ; a, 0,0)$ is presented in Appendix A. An alternative implementation of $\mathcal{F}_{\mathrm{GBessel2}}(x ; a, 0, p)$ is discussed in Appendix B. Inefficient Implementation of $\mathcal{F}_{23}(x ; a, d, 0)$ is presented in Appendix $\mathrm{C}$. Recursive implementation of $\mathcal{F}_{22}(x ; a, d, p)$ is given in Appendix D. In Appendix E, the Srivastava's triple hypergeometric series $F^{(3)}[x, y, z]$ is provided. In Appendix F, a super-efficient implementation of the Srivastava's triple hypergeometric series $F^{(3)}[x, y, z]$ is presented.

\section{Special Cases of the Generalized Bessel Function Distribution of the Second Kind PDFs}

From Progri 2016, (47) the generalized Bessel distribution of the second kind has its pdf given by

$$
f_{\mathrm{GBessel} 2}(x ; a, d, p)=\frac{|x|^{p_{e}} e^{-\frac{d}{a}|x|} K_{p}\left(\frac{|x|}{a}\right)}{C_{2}(p, d) a^{p_{2}}}
$$

for $-\infty<x<\infty, a>0, d \geq 0$, and $p>-1 / 2$ where $K_{p}(x)$ (see Gradshteyn and Ryzhik [24] pg. 917 ex. 8.432 3.) is given by Progri 2016, [1] (47) and $C_{2}(p, d)$ is given by Progri 2016 [1], (56)

$$
C_{2}(p, d)=\frac{\sqrt{\pi} 2^{p_{2}} \Gamma\left(2 p_{1}\right) \mathrm{F}\left(2 p_{1}, p_{1} ; p_{3} ; \frac{d-1}{d+1}\right)}{(d+1)^{2 p_{1} \Gamma\left(p_{3}\right)}}
$$

When $p>0$ then the Progri 2016 [1], (58)

$$
f_{\mathrm{GBessel} 2}(0 ; a, d, p>0)=\frac{2^{-2}(d+1)^{2 p_{1} \Gamma\left(p_{3}\right) \Gamma(p)}}{\sqrt{\pi} a \Gamma\left(2 p_{1}\right) \mathrm{F}\left(2 p_{1}, p_{1} ; p_{3} ; \frac{d-1}{d+1}\right)}
$$

However from Progri 2016 [1] we cannot know what happens to $f_{\mathrm{GBessel} 2}(0 ; a, d, p=0)$ and $f_{\mathrm{GBessel} 2}(0 ; a, d, p<$ $0)$ when $-1 / 2<p \leq 0$. The answer to these cases in given further down in the section.

Other important special cases consider when $p$ is an integer and is not an integer when $d=0$.

The special case when $d=0$ and $p=0$ is considered as a baseline special case because it serves as a test case for the other two special cases when: (1) $d=0$ and $p \neq 0, p$ a positive integer, which also serves as a test case for the special case when, (2) $d \neq 0$ and $p \neq 0, p$ a positive integer.

Moreover, the special case when $d=0$ and $p=0$ also serves as test case when $d \neq 0$ and $p=0$.

The special case when $d=0$ and $p \neq 0$ not an integer serves as a test case for the general case when $d \neq 0$ and $p \neq$ 
0 not an integer which expensively discussed in both Progri 2016 [1] and Progri 2018 [2].

For other values of $d<0$ and $p \leq-1 / 2$ for now we are going to assume that

$$
f_{\mathrm{GBessel} 2}(x ; a, d<0, p \leq-1 / 2)=0^{\mathrm{iii}}
$$

With that being said, in this section we consider five special cases of the generalized Bessel function distribution of the second kind.

\subsection{Special Case: $d=0, p \neq 0$ not an Integer}

If we set $d=0$ then from (1) we have

$$
f_{\mathrm{GBessel} 2}(x ; a, 0, p)=\frac{|x|^{p_{K}}\left(\frac{|x|}{a}\right)}{C_{2}(p, 0) a^{p_{2}}}
$$

Where $C_{2}(p, 0)($ see (2) and Progri 2016 [1], (67)-(70))

$$
C_{2}(p, 0)=\frac{\sqrt{\pi} 2^{p_{2}} \Gamma\left(2 p_{1}\right) \mathrm{F}\left(2 p_{1}, p_{1} ; p_{3} ;-1\right)}{\Gamma\left(p_{3}\right)} \equiv \sqrt{\pi} 2^{p} \Gamma\left(p_{1}\right)
$$

First, we need to develop the series expansion of the modified Bessel function of the second kind, $K_{p}(t)$, based on the series expansion of the modified Bessel function of the first kind $I_{p}(t)$ (see Arfken, G.B., and Weber, H.J., 1995, [25],

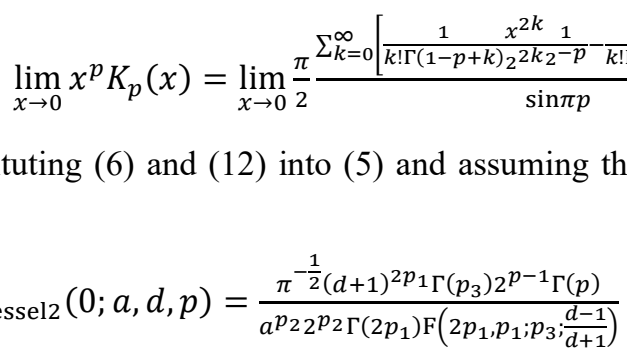

So if we set $d=0$ and $p>0$ yields

$$
\begin{aligned}
& f_{\mathrm{GBessel} 2}(0 ; a, 0, p>0)=\frac{\Gamma(p)}{\sqrt{\pi} 2 \Gamma\left(p_{1}\right) a^{p_{2}}} \\
& \lim _{x \rightarrow 0} x^{-q} K_{-q}(x)=\lim _{x \rightarrow 0} x^{-q} K_{-q}(x)=\lim _{x \rightarrow 0} \frac{\pi}{2} \frac{\sum_{k=0}^{\infty}\left[\frac{1}{k ! \Gamma(1-q+k)_{2}{ }^{2 k} 2^{-q}}-\frac{1}{k ! \Gamma\left(q_{2}+k\right)_{2}{ }^{2} 2^{2 k}}\right]}{\sin \pi q}
\end{aligned}
$$

(11.118) pg. 667 or Gradshteyn, Ryzhik, 2007 [24] pg. 928 ex. 8.485)

$$
K_{p}(x)=\frac{\pi}{2} \frac{I_{-p}(x)-I_{p}(x)}{\sin \pi p}
$$

where the series expansion of the modified Bessel function of the first kind, $I_{p}(t)$, (see Gradshteyn, Ryzhik, 2007 [24] pg. 919 ex. 8.445)

$$
\begin{aligned}
& I_{p}(x)=\sum_{k=0}^{\infty} \frac{1}{k ! \Gamma\left(p_{2}+k\right)} \frac{x^{p+2 k}}{2^{p+2 k}} \\
& I_{-p}(x)=\sum_{k=0}^{\infty} \frac{1}{k ! \Gamma(1-p+k)} \frac{x^{-p+2 k}}{2^{-p+2 k}}
\end{aligned}
$$

where $p$ is not an integer.

Substituting (8) and (9) into (7) yields

$$
K_{p}(x)=\frac{\pi}{2} \frac{\sum_{k=0}^{\infty} \frac{1}{k ! \Gamma(1-p+k)_{2}-p+2 k}-\sum_{k=0}^{\infty} \frac{1 \quad x^{p+2 k}}{k ! \Gamma\left(p_{2}+k\right)_{2} p+2 k}}{\sin \pi p}
$$

Which is equivalent with

$$
K_{p}(x)=\frac{\pi}{2} \frac{\sum_{k=0}^{\infty}\left[\frac{1}{\left.k ! \Gamma(1-p+k)_{2}^{2 k_{2}-p}-\frac{1}{k ! \Gamma\left(p_{2}+k\right) 2_{2} p_{2}^{2 k}}\right]}\right.}{\sin \pi p}
$$

If we take the limit of

$=\frac{\pi}{\Gamma(1-p) 2^{1-p} \sin \pi p}=2^{p-1} \Gamma(p)$

What happens when for $-1 / 2<p<0$ ? Which is equivalent with $0<q \equiv-p<1 / 2$. We already know that

$K_{q}(x)=\frac{\pi}{2} \frac{\sum_{k=0}^{\infty}\left[\frac{1}{\left.k ! \Gamma(1-q+k) 2^{2 k} 2^{2-q}-\frac{1}{k ! \Gamma(1+q+k) 2^{q}{ }^{2 k}}\right]}\right.}{\sin \pi q}=K_{-q}(x)$

If we take the limit of

Which is identical to

$$
\lim _{x \rightarrow 0} x^{-q} K_{-q}(x)=\lim _{x \rightarrow 0} x^{-q} K_{-q}(x)=\lim _{x \rightarrow 0} \frac{\pi}{2} \frac{\frac{1}{\Gamma(1-q) x^{-2 q}}-\frac{1}{2^{-q}}-\frac{1}{\Gamma\left(q_{2}\right) 2^{q}}}{\sin \pi q}=\infty
$$

Substituting (6) and (17) into (5) and for $p<0$ yields

$$
f_{\mathrm{GBessel} 2}(x ; a, 0, p=-q<0 \equiv q=-p>0)=\infty
$$

So if we set $d=0$ and $p<0$ yields

$$
f_{\mathrm{GBessel} 2}(x ; a, 0, p<0)=\infty
$$

This concludes the derivations of the special case for $d=0$ and $p \neq 0$. Next we consider the special case when both $d=$ 0 and $p=0$.

\subsection{Special Case: $d=0, p=0$}

If we set $d=0$ and $p=0$ then from (1) we have

$$
f_{\mathrm{GBesse} 2}(x ; a, 0,0)=\frac{K_{0}\left(\frac{|x|}{a}\right)}{C_{2}(0,0) a}
$$

When $p=0$ we have from (see Gradshteyn, Ryzhik, 2007 
[24] pg. 919 ex. 8.4451 . and 3.)

$$
K_{0}(x)=-\ln \left(\frac{x}{2}\right)\left[I_{0}(x) \equiv \sum_{k=0}^{\infty} \frac{x^{2 k}}{2^{2 k}(k !)^{2}}\right]+\sum_{k=0}^{\infty} \frac{x^{2 k}}{2^{2 k}(k !)^{2}} \psi(k+1)
$$

Which is equivalent with

$$
K_{0}(x)=\sum_{k=0}^{\infty} \frac{x^{2 k}\left[\psi(k+1)-\ln \left(\frac{x}{2}\right)\right]}{2^{2 k}(k !)^{2}}
$$

From $C_{2}(0,0)$ (see Progri 2016, (67)-(70))

$$
C_{2}(0,0)=\sqrt{\pi} \Gamma\left(\frac{1}{2}\right)=\pi
$$

If we take the limit

$$
\lim _{x \rightarrow 0} K_{0}(x)=\lim _{x \rightarrow 0} \sum_{k=0}^{\infty} \frac{x^{2 k}\left[\psi(k+1)-\ln \left(\frac{x}{2}\right)\right]}{2^{2 k}(k !)^{2}}=\infty
$$

Which is equivalent with

$$
\lim _{x \rightarrow 0} K_{0}(x)=\lim _{x \rightarrow 0} \sum_{k=0}^{\infty}\left[\psi(1)-\ln \left(\frac{x}{2}\right)\right]=\infty
$$

Substituting (23) and (25) into (20) yields

$$
f_{\mathrm{GBessel} 2}(0 ; a, 0,0)=\infty
$$

Hence, the pdf of $f_{\mathrm{GBessel} 2}(x ; a, 0,0)$ if given by

$$
f_{\mathrm{GBessel} 2}(x ; a, 0,0)=\mid \begin{array}{cc}
\frac{K_{0}\left(\frac{|x|}{a}\right)}{\pi a} & x \neq 0 \\
\infty & x=0
\end{array}
$$

This concludes the derivations of the special case for $d=0$ and $p=0$.

Next we consider the special case when $d=0$ and $p$ is a positive integer.

\subsection{Special Case: $d=0, p$ a Positive Integer}

If we set $d=0$ then from (1) we have

$$
f_{\mathrm{GBessel} 2}(x ; a, 0, p)=\frac{|x|^{p} K_{p}\left(\frac{|x|}{a}\right)}{c_{2}(p, 0) a^{p_{2}}}
$$

where $p \neq 0$ a positive integer or $\forall p \in \mathbb{Z}^{+}$and $C_{2}(p, 0)$ (see Progri 2016, (67)-(70)) and from a particular value of a gamma function we have

$$
C_{2}(p, 0)=\sqrt{\pi} 2^{p} \Gamma\left(p_{1}\right)=\sqrt{\pi} 2^{p} \frac{(2 p) !}{2^{2 p} p !} \sqrt{\pi}=\pi \frac{(2 p) !}{2^{p} p !}
$$

Is this the right answer? If we also set $p=0$ from (29) we get

$$
C_{2}(0,0)=\pi \frac{0 !}{2^{0} 0 !}=\pi
$$

Which is identical to (23) where we discussed the case for $p=0$ and $d=0$.

When $\forall p \in \mathbb{Z}^{+}$we have from (see Gradshteyn, Ryzhik, 2007 [24] pg. 919 ex. 8.446)

$$
K_{p}(x)=\frac{1}{2} \sum_{k=0}^{p-1} \frac{(-1)^{k}(p-k-1) !}{k !}\left(\frac{x}{2}\right)^{2 k-p}+(-1)^{p+1} \sum_{k=0}^{\infty} \frac{\ln \frac{x}{2}-\frac{\psi(k+1)+\psi(p+k+1)}{2}}{k !(p+k) !}\left(\frac{x}{2}\right)^{p+2 k}
$$

If we take the limit

$$
\lim _{x \rightarrow 0} \frac{x^{p} K_{p}\left(\frac{x}{a}\right)}{C_{2}(p, 0) a^{p_{2}}}=\lim _{x \rightarrow 0} \frac{x^{p}\left[\frac{1}{2} \sum_{k=0}^{p-1(-1)^{k}(p-k-1) !}\left(\frac{x}{2 a}\right)^{2 k-p}+(-1)^{p+1} \sum_{k=0}^{\infty} \frac{\ln \frac{x}{2 a}-\frac{\psi(k+1)+\psi(p+k+1)}{2}}{k !(p+k) !}\left(\frac{x}{2 a}\right)^{p+2 k}\right]}{C_{2}(p, 0) a^{p_{2}}}
$$

Which is equivalent with

$$
\lim _{x \rightarrow 0} \frac{x^{p} K_{p}\left(\frac{x}{a}\right)}{C_{2}(p, 0) a^{p_{2}}}=\lim _{x \rightarrow 0} \frac{\frac{(2 a)^{p}}{2}(p-1) !+(-1)^{p+1} \frac{x^{p}}{p !}\left(\frac{x}{2 a}\right)^{p}\left[\ln \frac{x}{2 a}-\frac{\psi(1)+\psi(p+1)}{2}\right]}{C_{2}(p, 0) a^{p_{2}}}=\frac{2^{2 p-1} p !(p-1) !}{\pi(2 p) ! a}=\frac{(p-1) !}{2 a \sqrt{\pi} \Gamma\left(p+\frac{1}{2}\right)}
$$

Hence, the pdf of $f_{\mathrm{GBessel} 2}(x ; a, 0, p)$ if given by

$$
f_{\mathrm{GBessel} 2}(x ; a, 0, p)=\mid \begin{array}{ll}
\frac{|x|^{p_{K}}\left(\frac{|x|}{a}\right)}{c_{2}(p, 0) a^{p_{2}}} & x \neq 0 \\
\frac{(p-1) !}{2 a \sqrt{\pi} \Gamma\left(p_{1}\right)} & x=0
\end{array}
$$

This concludes the derivations of the special case for $d=0$ and $p$ is a positive integer.

$$
f_{\mathrm{GBessel} 2}(x ; a, d, 0)=\frac{e^{-\frac{d}{a}|x|} K_{0}\left(\frac{|x|}{a}\right)}{a C_{2}(0, d)}
$$

for $-\infty<x<\infty, a>0$, and $d \geq 0$, where $K_{0}(x)$ (see Gradshteyn, Ryzhik, 2007 [24] pg. 919 ex. 8.445 1. and 3.) is given by Progri 2016 [1], (47).

From $C_{2}(0, d)$ (see Progri 2016 [1], (56))

$$
C_{2}(0, d)=\frac{4 \mathrm{~F}\left(1, \frac{1}{2} ; \frac{3}{2} ; \frac{d-1}{d+1}\right)}{d+1}
$$

\subsection{Special Case: $d \neq 0, p=0$}

When $d \neq 0$ and $p=0$ we have from (1) 
get (see Progri 2016 (69))

$$
C_{2}(0,0)=4 \mathrm{~F}\left(1, \frac{1}{2} ; \frac{3}{2} ;-1\right)=4 \frac{\Gamma^{2}\left(\frac{3}{2}\right)}{\Gamma(2)}=4\left(\frac{1}{2}\right)^{2} \frac{(\sqrt{\pi})^{2}}{\Gamma(2)}=\pi
$$

Which is identical to (23) where we discussed the case for $p=$ 0 and $d=0$. If we take the limit

$$
\lim _{x \rightarrow 0} e^{-\frac{d}{a}|x|} K_{0}(x)=\lim _{x \rightarrow 0} \sum_{k=0}^{\infty} \frac{x^{2 k}\left[\psi(k+1)-\ln \left(\frac{x}{2}\right)\right]}{2^{2 k}(k !)^{2}}=\infty
$$

Which is equivalent with

$$
\lim _{x \rightarrow 0} e^{-\frac{d}{a}|x|} K_{0}(x)=\lim _{x \rightarrow 0} \sum_{k=0}^{\infty}\left[\psi(1)-\ln \left(\frac{x}{2}\right)\right]=\infty
$$

Substituting (36) and (39) into (35) yields

$$
f_{\mathrm{GBessel} 2}(0 ; a, d, 0)=\infty
$$

Hence, the pdf of $f_{\mathrm{GBessel} 2}(x ; a, d, 0)$ if given by

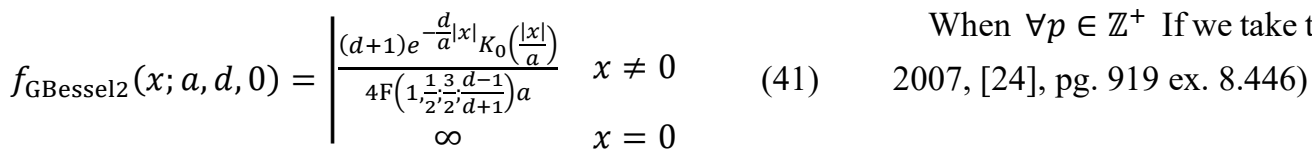
and $p=0$. and $p$ is a positive integer.

If we set $d \neq 0$ then from (1) we have gamma function we have

This concludes the derivations of the special case for $d \neq 0$

Next we consider the final (or last) special case when $d \neq 0$

\subsection{Special Case: $d \neq 0, p$ a Positive Integer}

$$
f_{\mathrm{GBessel} 2}(x ; a, d, p)=\frac{|x|^{p} e^{-\frac{d}{a}|x|} K_{p}\left(\frac{|x|}{a}\right)}{C_{2}(p, d) a^{p_{2}}}
$$

where $p \neq 0$ a positive integer or $\forall p \in \mathbb{Z}^{+}$and $C_{2}(p, d)$ (see Progri 2016 [1], (67)-(70)) and from a particular value of a

$$
C_{2}(p . d)=\frac{\sqrt{\pi} 2^{p_{2}} \Gamma\left(2 p_{1}\right) \mathrm{F}\left(2 p_{1}, p_{1} ; p_{3} ; \frac{d-1}{d+1}\right)}{(d+1)^{2} p_{1} \Gamma\left(p_{3}\right)}
$$

When $\forall p \in \mathbb{Z}^{+}$If we take the limit (see Gradshteyn, Ryzhik,

Which is equivalent with

$$
\lim _{x \rightarrow 0} \frac{x^{p} e^{-\frac{d}{a}|x|} K_{p}\left(\frac{x}{a}\right)}{C_{2}(p, d) a^{p_{2}}}=\lim _{x \rightarrow 0} \frac{\frac{(2 a)^{p}}{2}(p-1) !+(-1)^{p+1} \frac{x^{p}}{p !}\left(\frac{x}{2 a}\right)^{p}\left[\ln \frac{x}{2 a}-\frac{\psi(1)+\psi(p+1)}{2}\right]}{C_{2}(p, d) a^{p_{2}}}=\frac{(p-1) !(d+1)^{2 p_{1}} \Gamma\left(p_{3}\right)}{\sqrt{\pi} 4 \Gamma\left(2 p_{1}\right) \mathrm{F}\left(2 p_{1}, p_{1} ; p_{3} ; \frac{d-1}{d+1}\right) a}
$$

Hence, the pdf of $f_{\mathrm{GBessel} 2}(x ; a, 0, p)$ if given by

$$
f_{\mathrm{GBessel} 2}(x ; a, d, p)=\mid \begin{array}{cc}
\frac{|x|^{p} e^{-\frac{d}{a}|x|} K_{p}\left(\frac{|x|}{a}\right)}{C_{2}(p, d) a^{p_{2}}} & x \neq 0 \\
\frac{(p-1) !(d+1)^{2 p_{1} \Gamma\left(p_{3}\right)}}{\sqrt{\pi} 4 \Gamma\left(2 p_{1}\right) \mathrm{F}\left(2 p_{1}, p_{1} ; p_{3} ; \frac{d-1}{d+1}\right) a} & x=0
\end{array}
$$

This concludes the derivations of the special case for $d \neq 0$ and $p$ is a positive integer.

Next we consider the special cases the generalized Bessel distribution of the second kind CDFs.

\section{Special Cases of the Generalized Bessel Distribution of the Second Kind CDFs}

The special cases of the generalized Bessel distribution of the second kind CDFs follow from the discussion given in Sec. 2.

\subsection{Special Case: $d=0, p \neq 0$}

From the definition of the pdf of the generalized Bessel distribution of the second kind, we define its corresponding cdf as

$$
F_{\mathrm{GBessel} 2}(x ; a, 0, p)=\int_{-\infty}^{x} f_{\mathrm{GBessel} 2}(t ; a, 0, p) d t
$$

for $a>0, d=0$, and $p>-1 / 2, p \neq 0$, and $p$ not an integer, which can also be written as

$$
F_{\mathrm{GBessel} 2}(x ; a, 0, p)=\frac{\int_{-\infty}^{x}|t|^{p} K_{p}(|t|) d t}{C_{2}(p, 0)}
$$

where $C_{2}(p, 0)$ is given by (3).

Let us define

$$
\mathcal{F}_{\mathrm{GBessel} 2}(x ; a, 0, p)=\frac{\int_{0}^{x} t^{p} K_{p}(t) d t}{C_{2}(p, 0)}
$$

then

$$
F_{\mathrm{GBessel} 2}(x ; a, 0, p)=\frac{\begin{array}{ll}
1-2 \mathcal{F}_{\mathrm{GBessel} 2}(-x ; a, 0, p) & x<0 \\
1+2 \mathcal{F}_{\mathrm{GBessel} 2}(x ; a, 0, p) & x \geq 0
\end{array}}{2}
$$

Hence, if we compute the series expansion of (49); then, we have computed the series expansion of (50) via (49).

Substituting (7) into (48) yields

$$
\mathcal{F}_{\mathrm{GBessel} 2}(x ; a, 0, p)=\frac{\pi}{2} \frac{\int_{0}^{x} t^{p}\left[I_{-p}(t)-I_{p}(t)\right] d t}{C_{2}(p, 0) \sin \pi p}
$$

Or equivalently, 


$$
\mathcal{F}_{\mathrm{GBessel} 2}(x ; a, 0, p)=\frac{\pi}{2} \frac{\int_{0}^{x} t^{p} I_{-p}(t) d t-\int_{0}^{x} t^{p}{ } p(t) d t}{C_{2}(p, 0) \sin \pi p}
$$

Which is equivalent with

$$
\mathcal{F}_{\mathrm{GBessel} 2}(x ; a, 0, p)=\frac{\int_{0}^{x} t^{p_{I}}-p(t) d t-\int_{0}^{x} t^{p} I_{p}(t) d t}{\tilde{C}_{2}(p, 0)}
$$

Where

$$
\tilde{C}_{2}(p, 0)=\frac{2 C_{2}(p, 0) \sin \pi p}{\pi}=2^{p_{2}} \pi^{-\frac{1}{2}} \Gamma\left(p_{1}\right) \sin \pi p
$$

The second integral can be computed from using the series expansion of $I_{p}(t)$ (see Gradshteyn, Ryzhik, 2007 [24] pg. 919 ex. 8.445) we have

$\int_{0}^{x} t^{p} I_{p}(t) d t=\int_{0}^{x} t^{p} \sum_{k=0}^{\infty} \frac{1}{k ! \Gamma\left(p_{2}+k\right)} \frac{t^{p+2 k}}{2^{p+2 k}} d t$

Or by changing the order of summation and integration produces

$$
\int_{0}^{x} t^{p} I_{p}(t) d t=\sum_{k=0}^{\infty} \frac{\int_{0}^{x} t^{2 p+2 k} d t}{k ! \Gamma\left(p_{2}+k\right) 2^{p+2 k}}
$$

$$
\int_{0}^{x} t^{p} I_{p}(t) d t \equiv \frac{x^{2 p_{1}}}{2^{p}} \sum_{k=0}^{\infty} \frac{x^{2 k}}{k !\left(2 p_{1}+2 k\right) \Gamma\left(p_{2}+k\right) 2^{2 k}}
$$

Which is the same as

$$
\int_{0}^{x} t^{p} I_{p}(t) d t \equiv \frac{x^{2 p_{1}}}{p_{1} 2^{p_{2} \Gamma\left(p_{2}\right)}} \sum_{k=0}^{\infty} \frac{\left(p_{1}\right)_{k}}{\left(p_{3}\right)_{k}\left(p_{2}\right)_{k}} \frac{\left(x_{1}\right)^{k}}{k !}
$$

Which can be written by the help of Hypergeometric function [31]

$$
\int_{0}^{x} t^{p} I_{p}(t) d t \equiv \frac{x^{2 p_{1}}}{p_{1} 2^{p_{2} \Gamma\left(p_{2}\right)}}{ }_{1} F_{2}\left(p_{1} ; p_{3}, p_{2} ; x_{1}\right)
$$

Similarly, we compute the first integral using the series expansion of $I_{-p}(t)$ (see Gradshteyn, Ryzhik, 2007, [24] pg. 919 ex. 8.445) we have as follows

$$
\int_{0}^{x} t^{p} I_{-p}(t) d t=\int_{0}^{x} t^{p} \sum_{k=0}^{\infty} \frac{1}{k ! \Gamma(-p+k+1)} \frac{t^{-p+2 k}}{2^{-p+2 k}} d t
$$

Or by changing the order of summation and integration, performing some identities, and using the Hypergeometric function [31] produces

Which is identical to

$$
\int_{0}^{x} t^{p} I_{-p}(t) d t=\sum_{k=0}^{\infty} \frac{\int_{0}^{x} t^{2 k} d t}{k ! \Gamma(-p+k+1) 2^{-p+2 k}} \equiv \frac{x 2^{p}}{\Gamma(1-p)} \sum_{k=0}^{\infty} \frac{\left(\frac{1}{2}\right)_{k}}{\left(\frac{3}{2}\right)_{k}(1-p)_{k}} \frac{\left(x_{1}\right)^{k}}{k !}=\frac{x 2^{p}}{\Gamma(1-p)}{ }_{1} F_{2}\left(1 / 2 ; 3 / 2,1-p ; x_{1}\right)
$$

Substituting (59) and (61) into (53) yields

$$
\mathcal{F}_{\mathrm{GBessel} 2}(x ; a, 0, p)=\frac{\frac{x 2^{p}}{\Gamma(1-p)}{ }^{1} F_{2}\left(1 / 2 ; 3 / 2,1-p ; x_{1}\right)-\frac{x^{2 p_{1}}}{p_{1} 2^{p_{2} \Gamma\left(p_{2}\right)}{ }^{1} F_{2}}\left(p_{1} ; p_{3}, p_{2} ; x_{1}\right)}{\tilde{C}_{2}(p, 0)} \text { iv } ; x_{1}=\frac{x^{2}}{4}
$$

The question is as follows: "Can we obtain exactly the same

Let us try it if we can. By setting $d=0$ from Progri (2016 answer from Progri (2016 [1], (142)) by setting $d=0$ ?"

[1], (142)) we have

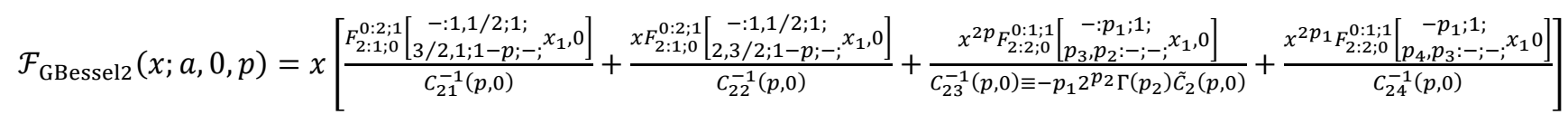

Where where $C_{2 i}(p, 0)$ for $i=\{1,2,3,4\}$ are given by

$$
\begin{aligned}
x_{1}=\frac{x^{2}}{4} ; x_{2}=\frac{d^{2} x^{2}}{4}= & 0 \\
C_{21}(p, 0) & =\frac{2^{p}}{\Gamma(1-p) \tilde{C}_{2}(p, 0)} ; C_{22}(p, 0)=\frac{2^{p-1} 0}{\Gamma(1-p) \tilde{C}_{2}(p, 0)}=0 ; C_{23}(p, 0)=\frac{-C_{11}(p, 0) C_{1}}{\tilde{C}_{2}(p, 0)} ; C_{24}(p, 0)=\frac{-C_{12}(p, 0) C_{1}}{\tilde{C}_{2}(p, 0)}=0 ;(6)
\end{aligned}
$$

Where $C_{11}(p, 0)$ and $C_{12}(p, 0)$ are given by Substituting (65) and (66) into (63) produces

$$
\begin{aligned}
& C_{11}(p, 0)=\frac{\Gamma\left(p_{2}\right)}{\Gamma\left(2 p_{2}\right)} ; C_{12}(p, 0)=\frac{C_{11}(p, d) 0}{2 p_{2}}=0(66) \\
& \mathcal{F}_{\mathrm{GBessel} 2}(x ; a, 0, p)=\frac{x F_{2: 1 ; 0}^{0: 2 ; 1}\left[\begin{array}{c}
-: 1,1 / 2 ; 1 ; \\
3 / 2,1 ; 1-p ;-;
\end{array} x_{1}, 0\right]}{C_{21}^{-1}(p, 0)}+\frac{x^{2 p+1} F_{2: 2 ; 0}^{0: 1 ; 1}\left[\begin{array}{c}
-: p_{1} ; 1 ; \\
p_{3}, p_{2}:-;-; ;
\end{array} x_{1}, 0\right]}{C_{23}^{-1}(p, 0) \equiv-p_{1} 2^{p_{2} \Gamma\left(p_{2}\right) \tilde{C}_{2}(p, 0)}}
\end{aligned}
$$

By comparing and contrasting (67) with (62) we arrive at the conclusion that (67) is identical to (62) if and only if

$$
F_{2: 1 ; 0}^{0: 2 ; 1}\left[\begin{array}{c}
-: 1,1 / 2 ; 1 ; \\
3 / 2,1 ; 1-p ;-;
\end{array} x_{1}, 0\right] \equiv{ }_{1} F_{2}\left[1 / 2 ; 3 / 2,1-p ; x_{1}\right]
$$


And

$$
F_{2: 2 ; 0}^{0: 1 ; 1}\left[\begin{array}{c}
-: p_{1} ; 1 ; \\
p_{3}, p_{2}:-;-;
\end{array} x_{1}, 0\right] \equiv{ }_{1} F_{2}\left(p_{1} ; p_{3}, p_{2} ; x_{1}\right)
$$

From the definition of the Kampé de Fériet function [11]-[13] we have

$$
F_{2: 1 ; 0}^{0: 2 ; 1}\left[\begin{array}{c}
-: 1,1 / 2 ; 1 ; \\
3 / 2,1 ; 1-p ;-;
\end{array} x_{1}, 0\right]=\sum_{k, m=0}^{\infty} \frac{(1)_{k}\left(\frac{1}{2}\right)_{k}}{\left(\frac{3}{2}\right)_{k+m}(1)_{k+m}(1-p)_{k}} \cdot \frac{x_{1}^{k}}{k !} \equiv \sum_{k=0}^{\infty} \frac{\left(\frac{1}{2}\right)_{k}}{\left(\frac{3}{2}\right)_{k}(1-p)_{k}} \cdot \frac{x_{1}^{k}}{k !} \equiv{ }_{1} F_{2}\left(\frac{1}{2} ; \frac{3}{2}, 1-p ; x_{1}\right)
$$

And

$$
F_{2: 2 ; 0}^{0: 1 ; 1}\left[\begin{array}{c}
-: p_{1} ; 1 ; \\
p_{3}, p_{2}:-;-;
\end{array} x_{1}, 0\right]=\sum_{k, m=0}^{\infty} \frac{\left(p_{1}\right)_{k}(1)_{m}}{\left(p_{3}\right)_{k+m}\left(p_{2}\right)_{k+m}} \cdot \frac{x_{1}^{k}}{k !} \equiv \sum_{k=0}^{\infty} \frac{\left(p_{1}\right)_{k}}{\left(p_{3}\right)_{k}\left(p_{2}\right)_{k}} \cdot \frac{x_{1}^{k}}{k !} \equiv_{1} F_{2}\left(p_{1} ; p_{3}, p_{2} ; x_{1}\right)
$$

This concludes the derivations of the special case for $d=0$ and $p \neq 0$. Next we consider the special case when both $d=$ 0 and $p=0$.

\subsection{Special Case: $d=0, p=0$}

From the definition of the pdf of the Generalized Bessel distribution of the second kind, we define its corresponding cdf as

$$
F_{\mathrm{GBessel} 2}(x ; a, 0,0)=\int_{-\infty}^{x} f_{\mathrm{GBessel} 2}(t ; a, 0,0) d t
$$

for $a>0, d=0$, and $p=0$, which can also be written as

$$
F_{\mathrm{GBessel} 2}(x ; a, 0,0)=\frac{\int_{-\infty}^{x} K_{0}(|t|) d t}{C_{2}(0,0)}
$$

where $C_{2}(0,0)$ is given by (18).

From (Gradshteyn, Ryzhik, 2007 [24],pg. 660, 6.5.11 12) we have

$$
\int_{0}^{\infty} K_{0}(t) d t=\frac{\pi}{2}
$$

Since,

$$
K_{0}(|t|)=\mid \begin{array}{cc}
K_{0}(t) & t>0 \\
\infty & t=0 \\
K_{0}(-t) & t<0
\end{array}
$$

We have

$$
\int_{-\infty}^{\infty} K_{0}(|t|) d t=\int_{-\infty}^{0} K_{0}(-t) d t+\int_{0}^{\infty} K_{0}(t) d t
$$

If we change the variables for the first integral we have

$\int_{-\infty}^{0} K_{0}(-t) d t=-\int_{0}^{\infty} K_{0}(t) d(-t)=\int_{0}^{\infty} K_{0}(t) d t=\frac{\pi}{2}$

Substituting (74) and (77) into (76) yields

$$
\int_{-\infty}^{\infty} K_{0}(|t|) d t=\frac{\pi}{2}+\frac{\pi}{2}=\pi
$$

Substituting (18) and (78) into (73) and setting $x=\infty$ yields

$$
F_{\mathrm{GBessel} 2}(\infty ; a, 0,0)=\frac{\int_{-\infty}^{\infty} K_{0}(|t|) d t}{C_{2}(0,0)}=\frac{\pi}{\pi}=1
$$

Let us define

$$
\mathcal{F}_{\mathrm{GBessel} 2}(x ; a, 0,0)=\frac{\int_{0}^{x} K_{0}(t) d t}{C_{2}(0,0)}
$$

then

$$
F_{\mathrm{GBessel} 2}(x ; a, 0,0)=\frac{\mid \begin{array}{ll}
1-2 \mathcal{F}_{\mathrm{GBessel} 2}(-x ; a, 0,0) & x<0 \\
1+2 \mathcal{F}_{\mathrm{GBessel} 2}(x ; a, 0,0) & x \geq 0
\end{array}}{2}
$$

Hence, if we compute the series expansion of (80); then, we have computed the series expansion of (81) via (80).

Substituting (18) and (21) into (80) we obtain the following

$$
\mathcal{F}_{\mathrm{GBessel} 2}(x ; a, 0,0)=\frac{\int_{0}^{x} \sum_{k=0}^{\infty} \frac{t^{2 k}\left[\psi(k+1)-\ln \left(\frac{t}{2}\right)\right]}{2^{2 k}(k !)^{2}} d t}{\pi}
$$

which is equivalent with by changing the order of summation and integration

$$
\mathcal{F}_{\mathrm{GBessel} 2}(x ; a, 0,0)=\frac{\sum_{k=0}^{\infty} \frac{\int_{0}^{x} t^{2 k}\left[\psi(k+1)-\ln \left(\frac{t}{2}\right)\right] d t}{2^{2 k}(k !)^{2}}}{\pi}
$$

which is the same as

$\mathcal{F}_{\mathrm{GBessel} 2}(x ; a, 0,0)=\frac{\sum_{k=0}^{\infty} \frac{\psi(k+1) \int_{0}^{x} t^{2 k} d t}{2^{2 k}(k !)^{2}}-\sum_{k=0}^{\infty} \frac{\int_{0}^{x} t^{2 k} \ln \left(\frac{t}{2}\right) d t}{2^{2 k}(k !)^{2}}}{\pi}$

The first integral is pretty straightforward to compute

$$
\int_{0}^{x} t^{2 k} d t=\frac{x^{2 k+1}}{2 k+1}
$$

Substituting (85) into the first summation of (84) yields

$$
\sum_{k=0}^{\infty} \frac{\psi(k+1) \int_{0}^{x} t^{2 k} d t}{2^{2 k}(k !)^{2}}=\sum_{k=0}^{\infty} \frac{\psi(k+1) \frac{x^{2 k+1}}{2 k+1}}{2^{2 k}(k !)^{2}}
$$

The second integral is more difficult to compute directly, so we integrate by parts

$$
\int_{0}^{x} \ln \left(\frac{t}{2}\right) t^{2 k} d t=\left.\ln \left(\frac{t}{2}\right) \frac{t^{2 k+1}}{2 k+1}\right|_{0} ^{x}-\int_{0}^{x} \frac{t^{2 k}}{2 k+1} d t
$$

The first part of the integral can be computed from

$$
\left.\ln \left(\frac{t}{2}\right) \frac{t^{2 k+1}}{2 k+1}\right|_{0} ^{x}=\ln \left(\frac{x}{2}\right) \frac{x^{2 k+1}}{2 k+1}-\lim _{x \rightarrow 0} \ln \left(\frac{x}{2}\right) \frac{x^{2 k+1}}{2 k+1}
$$


The lower limit of $\left.\ln \left(\frac{t}{2}\right) \frac{t^{2 k+1}}{2 k+1}\right|_{0} ^{x} \quad k=0,1, \cdots$ can be computed using L'Hôpital's rule [28]

$$
\lim _{x \rightarrow 0} \ln \left(\frac{x}{2}\right) \frac{x^{2 k+1}}{2 k+1}=\lim _{x \rightarrow 0} \frac{1}{(-2 k-1)} \frac{x^{2 k+1}}{(2 k+1)}=0
$$

The second integral is equal to

$$
\int_{0}^{x} \frac{t^{2 k}}{2 k+1} d t=\frac{x^{2 k+1}}{(2 k+1)^{2}}
$$

Substituting (89) into (88) and then (88) and (90) into (87) yields

$$
\int_{0}^{x} \ln \left(\frac{t}{2}\right) t^{2 k} d t=\ln \left(\frac{x}{2}\right) \frac{x^{2 k+1}}{2 k+1}-\frac{x^{2 k+1}}{(2 k+1)^{2}}
$$

Finally, substituting (86) and (91) into (84) we obtain the following

$$
\mathcal{F}_{\mathrm{GBessel} 2}(x ; a, 0,0)=\frac{\sum_{k=0}^{\infty} \frac{\psi(k+1) \frac{x^{2 k+1}}{2 k+1}}{2^{2 k}(k !)^{2}}-\sum_{k=0}^{\infty} \frac{\left[\ln \left(\frac{x}{2}\right) \frac{x^{2 k+1}}{2 k+1}-\frac{x^{2 k+1}}{(2 k+1)^{2}}\right]}{2^{2 k}(k !)^{2}}}{\pi}=\frac{\sum_{k=0}^{\infty} \frac{\psi(k+1) \frac{x^{2 k+1}}{2 k+1}}{2^{2 k}(k !)^{2}}-\ln \left(\frac{x}{2}\right) \sum_{k=0}^{\infty} \frac{\frac{x^{2 k+1}}{2 k+1}}{2^{2 k}(k !)^{2}}+\sum_{k=0}^{\infty} \frac{x^{2 k+1}}{\pi}(2 k+1)^{2}(k !)^{2}}{\pi}
$$

which is identical to

$$
\mathcal{F}_{\text {GBessel } 2}(x ; a, 0,0)=\mathcal{F}_{21}(x ; a, 0,0)+\mathcal{F}_{22}(x ; a, 0,0)+\mathcal{F}_{23}(x ; a, 0,0)
$$

Let us exploit these summations one at a time. We start with generalized hypergeometric function [31] the second summation can be expressed in terms of the

$$
\mathcal{F}_{22}(x ; a, d, 0)=\frac{-\ln \left(\frac{x}{2}\right)\left[\sum_{k=0}^{\infty} \frac{\frac{x^{2 k+1}}{2 k+1}}{2^{2 k}(k !)^{2}} \equiv x \sum_{k=0}^{\infty} \frac{\frac{x^{2 k}}{2^{2 k}}}{(2 k+1)(1)_{k} k !} \equiv x \sum_{k=0}^{\infty} \frac{\frac{x^{2 k}}{2^{2 k}}}{2\left(k+\frac{1}{2}\right)(1)_{k} k !} \equiv x \sum_{k=0}^{\infty} \frac{\left(\frac{1}{2}\right)_{k}\left(x_{1} \equiv \frac{x^{2}}{2^{2}}\right)^{k}}{\left(\frac{3}{2}\right)_{k}(1)_{k} k !} \equiv x_{1} F_{2}\left(\frac{1}{2} ; \frac{3}{2}, 1 ; x_{1}\right)\right]}{\pi}
$$

The third summation can be expressed in terms of the generalized hypergeometric function [31] as

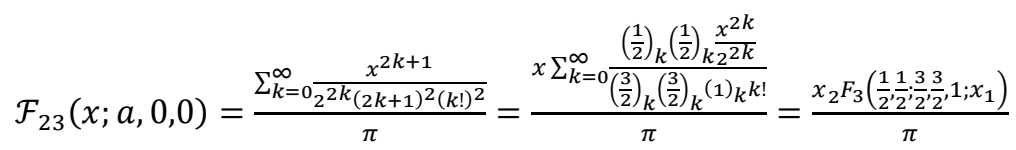

Now, we go back to compute the first summation

$$
\mathcal{F}_{21}(x ; a, 0,0)=\frac{\sum_{k=0}^{\infty} \frac{\psi(k+1) \frac{x^{2 k+1}}{2 k+1}}{2^{2 k}(k !)^{2}}}{\pi}=\frac{x \sum_{k=0}^{\infty} \frac{\psi(k+1)\left(\frac{1}{2}\right)_{k} x_{1}^{k}}{\left(\frac{3}{2}\right)_{k}(1) k_{k} k !}}{\pi}
$$

In appendix A we consider an inefficient implementation of $\mathcal{F}_{21}(x ; a, 0,0)$.

The digamma function is computed recursively $\psi(k+1)$

$$
\psi(k+1)=\frac{1}{k}+\psi(k)
$$

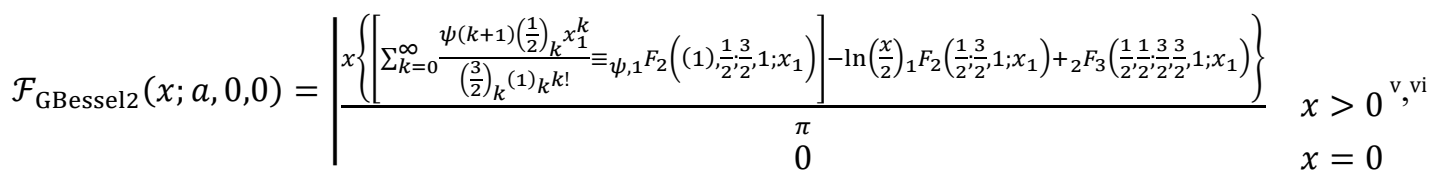

Equation (98) is an original expression not published anywhere else; however, care must be shown in the computation of the $\mathcal{F}_{21}(x ; a, 0,0)$.

This concludes the derivations of the special case for $d=0$ and $p=0$.

Next we consider the special case when for $d=0$ and $p$ a positive integer.
Therefore, $\mathcal{F}_{21}(x ; a, 0,0)$ can be computed recursively very efficiently and accurately.

Finally, Dr. Progri has successfully derived for the first time the cdf of the Bessel function distribution of the second kind for values of $p=0$ and $d=0$ which can be written with the help of the generalized hypergeometric function [31] and a special summation as follows

\subsection{Special Case: $d=0, p \neq 0$ a Positive Integer}

There are two approaches that are equivalent in both performance and speed to solve this special case: one is the recursive approach discussed here, the other is the direct approach discussed in Appendix B.

In order to compute the $\mathcal{F}_{\mathrm{GBessel} 2}(x ; a, 0, p)$ recursively, we 
will have to employ some properties of the modified Bessel function of the second kind (see Gradshteyn, Ryzhik, 2007, [24], pg. 929 ex. 8.486 10. and 11.)

$$
\begin{aligned}
& K_{p+1}(t)=K_{p-1}(t)+\frac{2 p}{t} K_{p}(t) ; p=1,2, \cdots, \infty \\
& \mathcal{F}_{\mathrm{GBessel} 2}\left(x ; a, d, p_{2}\right)=\frac{\int_{0}^{x} t^{p_{2}} K_{p_{2}}(t) d t}{C_{2}\left(p_{2}, 0\right)}=\frac{\int_{0}^{x} t^{p_{2}} K_{p-1}(t) d t+2 p \int_{0}^{x} t^{p} K_{p}(t) d t}{C_{2}\left(p_{2}, 0\right)}=\frac{\int_{0}^{x} t^{p_{2}} K_{p-1}(t) d t}{C_{2}\left(p_{2}, 0\right)}+\frac{p}{p_{1}} \mathcal{F}_{\mathrm{GBessel} 2}(x ; a, d, p)
\end{aligned}
$$

Next, let us evaluate the integral by parts and substituting (101) into the integration

$$
\int_{0}^{x} t^{p_{2}} K_{p-1}(t) d t=-\int_{0}^{x} t^{p_{2}} K_{p_{2}}(t) d t-2 \int_{0}^{x} t^{p_{2}} \frac{d K_{p}(t)}{d t} d t=-\frac{\mathcal{F}_{\mathrm{GBessel} 2}\left(x ; a, d, p_{2}\right)}{C_{2}^{-1}\left(p_{2}, 0\right)}-\left.2 t^{p_{2}} K_{p}(t)\right|_{0} ^{x}+2 p_{2} \int_{0}^{x} t^{p} K_{p}(t) d t
$$

Next, substituting (102) into (101) we obtain the recursive

$$
\mathcal{F}_{\text {GBessel2 }}(x ; a, 0, p) d=0, p \neq 0 \text { a positive Integer }
$$

algorithm to compute fast, accurate, and very efficiently, the

$$
\mathcal{F}_{\mathrm{GBessel} 2}\left(x ; a, d, p_{2}\right)=\mathcal{F}_{\mathrm{GBessel} 2}(x ; a, d, p)-\frac{x^{p_{2}} K_{p}(x)}{C_{2}\left(p_{2}, 0\right)} \rightarrow \mathcal{F}_{\mathrm{GBessel} 2}(x ; a, d, p)=\sum_{i=0}^{p-1}\left[\mathcal{F}_{\mathrm{GBessel} 2}(x ; a, d, i)-\frac{x^{i+1} K_{i}(x)}{C_{2}(i+1,0)}\right](10
$$

To know whether (102) is correct, we test it for $p=0$

$$
\mathcal{F}_{\mathrm{GBessel} 2}(x ; a, 0,0)=\left\{\begin{array}{cl}
\frac{\left.x\left\{\sum_{k=0}^{\infty} \frac{\psi(k+1)\left(\frac{1}{2}\right)_{k} x_{1}^{k}}{\left(\frac{3}{2}\right)_{k}(1)_{k} k !} \equiv_{\psi, 1} F_{2}\left((1), \frac{1}{2} ; \frac{3}{2}, 1 ; x_{1}\right)\right]-\ln \left(\frac{x}{2}\right)_{1} F_{2}\left(\frac{1}{2} ; \frac{3}{2}, 1 ; x_{1}\right)+{ }_{2} F_{3}\left(\frac{1}{2}, \frac{1}{2} ; \frac{3}{2}, \frac{3}{2}, 1 ; x_{1}\right)\right\}}{\pi} & x>0 \\
0 & x=0
\end{array}\right.
$$

Equation (104) is identical to (98) thus completing the proof of this test point.

This concludes the derivations of the special case for $d=0$ and $p$ is a positive integer.

Next we consider the special case when for $d \neq 0$ and $p=$ 0 .

\subsection{Special Case: $d \neq 0, p=0$}

When $d \neq 0$ and $p=0, f_{\mathrm{GBessel} 2}(x ; a, d, 0)$ is given by (41).

Following from Progri 2016 [1], (50)-(53), substituting (21) into (121) of Progri 2016 [1]

$$
\mathcal{F}_{\text {GBessel2 }}(x ; a, d, 0)=\frac{\int_{0}^{x} e^{-t d} \sum_{k=0}^{\infty} \frac{t^{2 k}\left[\psi(k+1)-\ln \left(\frac{t}{2}\right)\right]}{2^{2 k}(k !)^{2}} d t}{C_{2}(0, d)}
$$

which is equivalent with by changing the order of summation and integration

$$
\mathcal{F}_{\text {GBessel2 }}(x ; a, d, 0)=\frac{\sum_{k=0}^{\infty} \frac{\int_{0}^{x} t^{2 k} e^{-t d}\left[\psi(k+1)-\ln \left(\frac{t}{2}\right)\right] d t}{2^{2 k}(k !)^{2}}}{C_{2}(0, d)}
$$

which is identical to

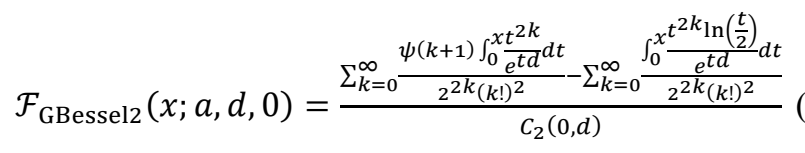

The first integral is pretty straightforward to compute (see Progri 2016 [1], (132))

$$
\int_{0}^{x} t^{2 k} e^{-t d} d t=\frac{\gamma(2 k+1, d x)}{d^{2 k+1}}
$$

Substituting (108) into the first summation of (107) yields

$$
\sum_{k=0}^{\infty} \frac{\psi(k+1) \int_{0}^{x} t^{2 k} e^{-t d} d t}{2^{2 k}(k !)^{2}}=\sum_{k=0}^{\infty} \frac{\psi(k+1) \frac{\gamma(2 k+1, d x)}{d^{2 k+1}}}{2^{2 k}(k !)^{2}} \mathrm{vii}
$$

Before computing the first summation of (109), first, from Progri, 2018 [2], (19) for $p=0$ we have

$$
\gamma(2 k+1, d x)=\frac{\left[\sum_{m=0}^{\infty} \frac{x_{2}^{m}}{\left(k+\frac{3}{2}\right)_{m}{ }^{(k+1) m}}+\frac{d x \sum_{m=0}^{\infty} \frac{x_{2}^{m}}{(k+2)_{m}\left(k+\frac{3}{2}\right)_{m}}}{2(k+1)}\right]}{(d x)^{-2 k-1} e^{d x}(2 k+1)}
$$

Substituting (110) into the first summation of (107) yields,

$$
\mathcal{F}_{21}(x ; a, d, 0)=\frac{\sum_{k=0}^{\infty} \frac{\psi(k+1) \frac{x^{2 k+1} e^{-d x}}{(2 k+1)}\left[\sum_{m=0}^{\infty} \frac{x_{2}^{m}}{\left(k+\frac{3}{2}\right)_{m}^{(k+1) m}}+\frac{d x}{2(k+1)} \sum_{m=0}^{\infty} \frac{x_{2}^{m}}{2^{2 k}(k+2) m\left(k+\frac{3}{2}\right)_{m}}\right]}{C_{2}(0, d)}}{C^{2}}
$$

Which is equivalent with 


$$
\mathcal{F}_{21}(x ; a, d, 0)=\frac{x e^{-d x}\left[\sum_{k, m=0}^{\infty} \frac{\psi(k+1)\left(\frac{1}{2}\right)_{k}(1) m_{1} \frac{x_{1}^{k} x_{2}^{m}}{k ! m !}}{\left(\frac{3}{2}\right)_{k+m}{ }^{(1)} k+m}+\frac{d x}{2} \sum_{k, m=0}^{\infty} \frac{\psi(k+1)\left(\frac{1}{2}\right)_{k}(1) m \frac{x_{1}^{k} x_{2}^{m}}{k ! m !}}{(2)_{m k+}\left(\frac{3}{2}\right)_{k+m}}\right]}{C_{2}(0, d)}
$$

Equation (112) can be written with the help of the modified generalized Kampé de Fériet function [11]-[13] as follows

$$
\sum_{k=0}^{\infty} \frac{\psi(k+1) \frac{\gamma(2 k+1, d x)}{d^{2 k+1}}}{2^{2 k}(k !)^{2}}=x e^{-d x}\left[F_{2: 0 ; 0}^{0: \psi, 1 ; 1}\left[\begin{array}{c}
-:(1), 1 / 2 ; 1 ; x_{1}, x_{2} \\
3 / 2,1:-;-;
\end{array}\right]+\frac{d x}{2} F_{2: 0 ; 0}^{0: \psi, 1 ; 1}\left[\begin{array}{c}
-:(1), 1 / 2 ; 1 ; x_{1}, x_{2} \\
2,3 / 2:-;-;
\end{array}\right]\right]
$$

Substituting (113) into (109) produces the final expression of $\quad \mathcal{F}_{21}(x ; a, d, 0)$

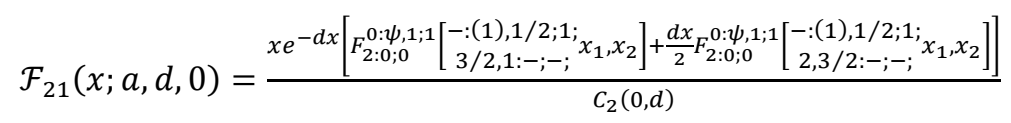

Equation (114) eliminates a singularity for $d=0$, which the recursive approach was not able to; hence, (114) is much better implementation than the recursive algorithm described in the endnote viii.

$$
\int_{0}^{x} \ln \left(\frac{t}{2}\right) t^{2 k} e^{-t d} d t=\left[\left.\ln \left(\frac{t}{2}\right) \frac{\gamma(2 k+1, t d)}{d^{2 k+1}}\right|_{0} ^{x}=\ln \left(\frac{x}{2}\right) \frac{\gamma(2 k+1, d x)}{d^{2 k+1}}-\lim _{x \rightarrow 0} \ln \left(\frac{x}{2}\right) \frac{\gamma(2 k+1, d x)}{d^{2 k+1}}\right]-\int_{0}^{x} \frac{t^{-1} \gamma(2 k+1, t d)}{d^{2 k+1}} d t
$$

The lower limit of $\ln (x / 2) \gamma(2 k+1, d x) / d^{2 k+1} \quad k=$ [28]

$0,1, \cdots$ can be computed using L'Hôpital's rule (see also (89))

$$
\lim _{x \rightarrow 0} \ln \left(\frac{x}{2}\right) \frac{\gamma(2 k+1, d x)}{d^{2 k+1}}=\lim _{x \rightarrow 0} \ln \left(\frac{x}{2}\right) \frac{d^{2 k+1} x^{2 k+1} e^{-d x} \sum_{n=0}^{\infty} \frac{(1)_{n}(d x)^{n}}{(2+2 k)_{n}} n !}{(2 k+1)}=\frac{d^{2 k+1} \lim _{x \rightarrow 0} \ln \left(\frac{x}{2}\right) x^{2 k+1}}{(2 k+1)}=0
$$

Substituting (115), (116) into the second summation of (107) yields

$$
\mathcal{F}_{22}(x ; a, d, 0)+\mathcal{F}_{23}(x ; a, d, 0)=\frac{\sum_{k=0}^{\infty} \frac{\int_{0}^{x^{2 k} \frac{t^{2 k} \ln \left(\frac{t}{2}\right)}{e^{t}} d t}}{2^{2 k}(k !)^{2}}}{C_{2}(0, d)}=\frac{\ln \left(\frac{x}{2}\right) \sum_{k=0}^{\infty} \frac{\frac{\gamma(2 k+1, d x)}{d^{2 k+1}}}{2^{2 k}(k !)^{2}}-\sum_{k=0}^{\infty} \frac{\int_{0}^{x} \frac{t^{-1} \gamma(2 k+1, t d)}{d^{2 k+1}} d t}{2^{2 k}(k !)^{2}}}{C_{2}(0, d)}
$$

We start with the second summation. First, substituting (110) into the first summation yields

$$
\sum_{k=0}^{\infty} \frac{\frac{\gamma(2 k+1, d x)}{d^{2 k+1} 1_{2} k}}{(k !)^{2}}=x e^{-d x}\left[\sum_{k, m=0}^{\infty} \frac{\left(\frac{1}{2}\right)_{k}(1)_{m}}{\left(\frac{3}{2}\right)_{k+m}(1)_{k+m}} \frac{x_{1}^{k}}{k !} \frac{x_{2}^{m}}{m !}+\frac{d x}{4} \sum_{k, m=0}^{\infty} \frac{\left(\frac{1}{2}\right)_{k}(1)_{m}}{(2)_{k+m}\left(\frac{3}{2}\right)_{k+m}} \frac{x_{1}^{k}}{k !} \frac{x_{2}^{m}}{m !}\right]
$$

Equation (118) can be written with the help of the Kampé de Fériet function [11]-[13] as follows

$$
\sum_{k=0}^{\infty} \frac{\frac{\gamma(2 k+1, d x)}{d^{2 k+1}}}{2^{2 k}(k !)^{2}}=x e^{-d x}\left[F_{2: 0 ; 0}^{0: 1 ; 1}\left[\begin{array}{c}
-: 1 / 2 ; 1 ; \\
3 / 2,1:-;-;
\end{array} x_{1}, x_{2}\right]+\frac{d x}{2} F_{2: 0 ; 0}^{0: 1 ; 1}\left[\begin{array}{c}
-: 1 / 2 ; 1 ; \\
2,3 / 2:-;-;
\end{array} x_{1}, x_{2}\right]\right]
$$

Substituting (119) into the second summation yields,

$$
\mathcal{F}_{22}(x ; a, d, 0)=\frac{-\ln \left(\frac{x}{2}\right) \sum_{k=0}^{\infty} \frac{\frac{\gamma(2 k+1, d x)}{d^{2 k+1}}}{2^{2 k}(k !)^{2}}}{C_{2}(0, d)}=\frac{-\ln \left(\frac{x}{2}\right) x e^{-d x}\left[F_{2: 0 ; 0}^{0: 1 ; 1}\left[\begin{array}{c}
-: 1 / 2 ; 1 ; \\
3 / 2,1:-;-;
\end{array} x_{1}, x_{2}\right]+\frac{d x}{2} F_{2: 0 ; 0}^{0: 1 ; 1}\left[\begin{array}{c}
-: 1 / 2 ; 1 ; \\
2,3 / 2:-;-;
\end{array} x_{1}, x_{2}\right]\right]}{C_{2}(0, d)}
$$

For the second summation of (119) we employ the series performing the integration we obtain expansion of the incomplete gamma function and after

$$
\mathcal{F}_{23}(x ; a, d, 0)=\frac{\sum_{k=0}^{\infty} \frac{\sum_{n=0}^{\infty} \frac{(-1)^{n} d^{n} \frac{x^{2 k+n+1}}{(2 k+n+1)^{2}}}{n !}}{2^{2 k}(k !)^{2}}}{C_{2}(0, d)}=\frac{x \sum_{k, n=0}^{\infty} \frac{\frac{\frac{x^{2 k}}{2^{2 k}}(-d x)^{n}}{n !}(2 k+n+1)^{2} k !}{C_{2}(0, d)}}{C^{2}}
$$

Next, we perform the expansion into even and odd terms we have 


$$
\mathcal{F}_{23}(x ; a, d, 0)=\frac{x \sum_{k, m=0}^{\infty} \frac{\frac{x_{1}^{k}(-d x)^{2 m}}{k !(2 m) !}}{(2 k+2 m+1)^{2} k !}+x \sum_{k, m=0}^{\infty} \frac{\frac{x_{1}^{k}(-d x)^{2 m+1}}{k !(2 m+1) !}}{(2 k+2 m+1+1)^{2} k !}}{C_{2}(0, d)}
$$

Which is equivalent with

$$
\mathcal{F}_{23}(x ; a, d, 0)=\frac{x \sum_{k, m=0}^{\infty} \frac{\frac{x_{1}^{k}(-d x)^{2 m}}{k !\left(\frac{1}{2}\right) m^{22 m} m !}}{(2 k+2 m+1)^{2} k !}+d x^{2} \sum_{k, m=0}^{\infty} \frac{\frac{x_{1}^{k}(-d x)^{2 m}}{k !(2 m+1)\left(\frac{1}{2}\right) m_{m}^{2^{2 m} m !}}}{(2 k+2 m+2)^{2} k !}}{C_{2}(0, d)}
$$

Which is identical to

$$
\mathcal{F}_{23}(x ; a, d, 0)=\frac{\frac{1}{4} x \sum_{k, m=0}^{\infty} \frac{\frac{x_{1}^{k} x_{2}^{m}}{k !\left(\frac{1}{2}\right)_{m}^{m !}}}{\left(k+m+\frac{1}{2}\right)^{2} k !}-\frac{d x^{2}}{8} \sum_{k, m=0}^{\infty} \frac{\frac{x_{1}^{k}}{k !\left(m+\frac{1}{2}\right)\left(\frac{1}{2}\right)_{m}^{m} m !}}{(k+m+1)^{2} k !}}{C_{2}(0, d)}
$$

Equations (124) can be written as follows

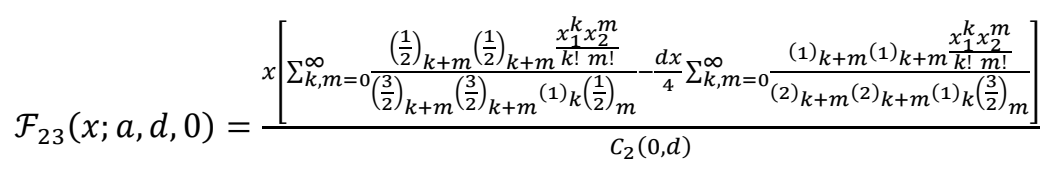

Finally, Dr. Progri has successfully derived for the first time Kampé de Fériet function [11]-[13] as follows that this summation which can be written with the help of the

$$
\mathcal{F}_{23}(x ; a, d, 0)=\frac{x\left[F_{2: 1 ; 1}^{2: 0 ; 0}\left[\begin{array}{c}
1 / 2,1 / 2:-;-; \\
3 / 2,3 / 2: 1 ; 1 / 2 ;
\end{array} x_{1}, x_{2}\right]-\frac{d x}{4} F_{2: 1 ; 1}^{2: 0 ; 0}\left[\begin{array}{c}
1,1:-;-; \\
2,2: 1 ; 3 / 2 ;
\end{array} x_{1}, x_{2}\right]\right]}{C_{2}(0, d)}
$$

Finally, substituting (114), (120), and (126) into (107) $\mathcal{F}_{\mathrm{GBessel}}(x ; a, d \neq 0,0)$ produces the desired closed form expression of

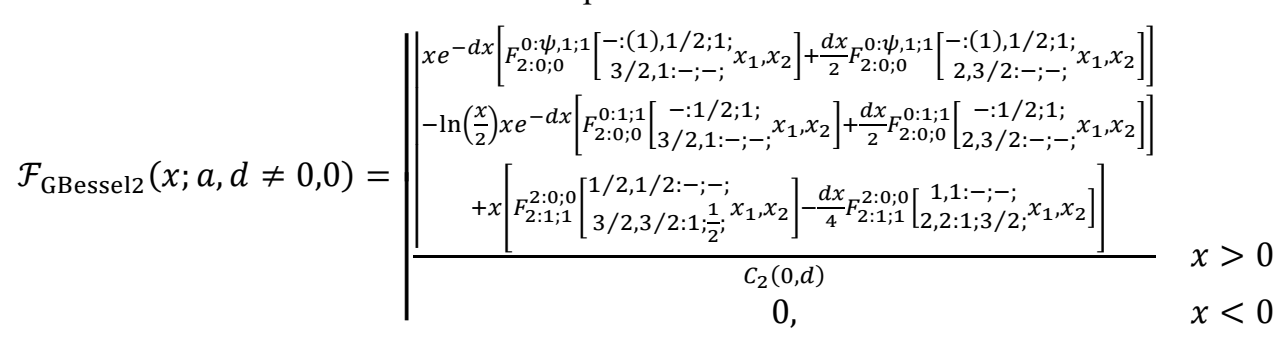

Equation (127) is an original expression not published anywhere else; however, care must be shown in the computation of the Kampé de Fériet function [2].

The obvious question is as follows. Is (127) the right

$$
\mathcal{F}_{\mathrm{GBesse} 2}(x ; a, 0,0)=\frac{x F_{2: 0 ; 0}^{0: 1 ; 1}\left[\begin{array}{c}
-: \psi(1), 1 / 2 ; 1 ; \\
3 / 2,1:-;-;
\end{array} x_{1}, x_{2}\right]-x \ln \left(\frac{x}{2}\right) F_{1: 1 ; 1}^{1: 0 ; 0}\left[\begin{array}{c}
1 / 2:-;-; \\
3 / 2: 1 ; 1 / 2 ;
\end{array} x_{1}, x_{2}\right]+\left.x F_{2: 1 ; 1}^{2: 0 ; 0}\left[\begin{array}{c}
1 / 2,1 / 2:-;-; \\
3 / 2,3 / 2: 1 ; 1 / 2 ;
\end{array} x_{1}, x_{2}\right]\right|_{d=0}}{C_{2}(0,0)}
$$

Evaluating the two Kampé de Fériet function [11]-[13] at $\quad d=0$ yields

$$
\begin{aligned}
& F_{2: 0 ; 0}^{0: 1 ; 1}\left[\left.\begin{array}{c}
-: \psi(1), 1 / 2 ; 1 ; x_{1}, x_{2} \\
3 / 2,1:-;-;
\end{array}\right|_{d=0}=\sum_{k, m=0}^{\infty} \frac{\psi(1+k)\left(\frac{1}{2}\right)_{k}(1)_{m}}{\left(\frac{3}{2}\right)_{k+m}(1)_{k+m}} \frac{x_{1}^{k}}{k !} \frac{0^{m}}{m !}=\sum_{k=0}^{\infty} \frac{\psi(1+k)\left(\frac{1}{2}\right)_{k} \frac{x_{1}^{k}}{k !}}{\left(\frac{3}{2}\right)_{k}(1)_{k}}={ }_{\psi, 1} F_{2}\left[(1) 1 / 2 ; 3 / 2,1 ; x_{1}\right](12\right. \\
& \left.F_{2: 0 ; 0}^{0: 1,1}\left[\begin{array}{l}
-: 1 / 2 ; 1 ; \\
3 / 2,1:-;-;
\end{array} x_{1}, x_{2}\right]\right|_{d=0}=\sum_{k, m=0}^{\infty} \frac{\left(\frac{1}{2}\right)_{k}(1)_{m}}{\left(\frac{3}{2}\right)_{k+m}(1)_{k+m}} \frac{x_{1}^{k}}{k !} \frac{0^{m}}{m !}=\sum_{k=0}^{\infty} \frac{\left(\frac{1}{2}\right)_{k} \frac{x_{1}^{k}}{\left(\frac{3}{2}\right)_{k}(1)_{k}}}{m}={ }_{1} F_{2}\left(1 / 2 ; 3 / 2,1 ; x_{1}\right)
\end{aligned}
$$




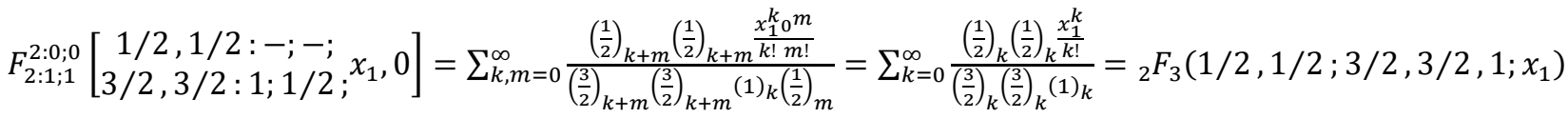

Substituting (129)-(131) into (128) yields

$$
\mathcal{F}_{\text {GBessel } 2}(x ; a, 0,0)=x \frac{\psi, 1 F_{2}\left[(1) 1 / 2 ; 3 / 2,1 ; x_{1}\right]-\ln \left(\frac{x}{2}\right){ }_{1} F_{2}\left(1 / 2 ; 3 / 2,1 ; x_{1}\right)+{ }_{2} F_{3}\left(1 / 2,1 / 2 ; 3 / 2,3 / 2,1 ; x_{1}\right)}{C_{2}(0,0) \equiv \pi}
$$

Equation (132) is identical to (98); thus, completing the proof for $d=0$.

This concludes the derivations of the special case for $d \neq 0$ and $p=0$.

Next we consider some remarks the most important special case when $d \neq 0$ and $p$ is a positive integer.

\subsection{Special Case: $d \neq 0, p$ a Positive Integer}

When $d \neq 0$ and $p$ a positive, $f_{\mathrm{GBessel} 2}(x ; a, d, p)$ is given by (46).

Following from Progri 2016 [1], (50)-(53), substituting (31) into (121) of Progri 2016 [1]

$$
\mathcal{F}_{\text {GBessel2 }}(x ; a, d, p)=\frac{\int_{0}^{x} t^{p} e^{-t d}\left[\frac{1}{2} \sum_{k=0}^{p-1(-1)^{k}(p-k-1) !} \frac{t}{k}\right)^{2 k-p}+(-1)^{p} \sum_{k=0}^{\infty} \frac{\frac{\psi(k+1)+\psi(p+k+1)}{2}-\ln \frac{t}{2}}{\left.k !\left(\frac{t}{2}\right)^{p+2 k}\right]} d t}{C_{2}(p, d)}
$$

which is equivalent with by changing the order of summation and integration

$$
\mathcal{F}_{\mathrm{GBessel} 2}(x ; a, d, p)=\frac{2^{p-1} \sum_{k=0}^{p-1} \frac{(-1)^{k}(p-k-1) ! \int_{0}^{x} e^{-t d} t^{2 k} d t}{k ! 2^{2 k}}+\frac{(-1) p}{2^{p}{ }^{p}} \sum_{k=0}^{\infty} \frac{[\psi(k+1)+\psi(p+k+1)] \int_{0}^{x} e^{-t d} t^{2 p+2 k} d t}{k !(p+k) ! 2^{2 k}}+\frac{(-1) p_{2}}{2^{p}} \sum_{k=0}^{\infty} \frac{\int_{0}^{x} \ln \frac{t}{e^{-}}-t d t^{2 p+2 k} d t}{k !(p+k) ! 2^{2 k}}}{C_{2}(p, d)}
$$

The first integral is pretty straightforward to compute (see 108).

We start with the first summation. Substituting, (110) into the

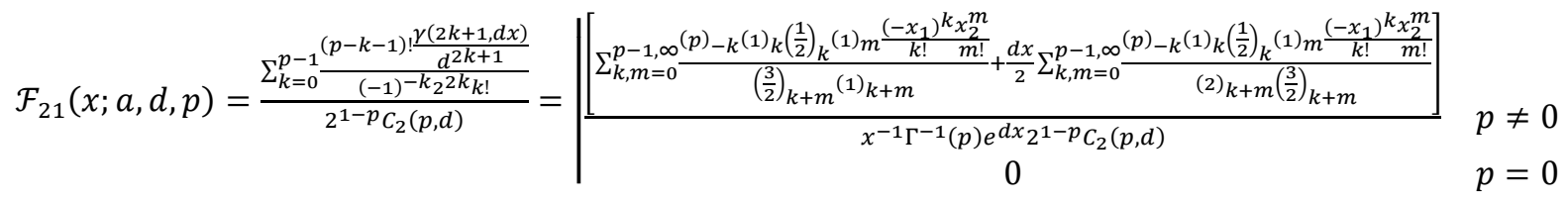

Equation (135) can be written with the help of the generalized Kampé de Fériet function [11]-[13] as follows

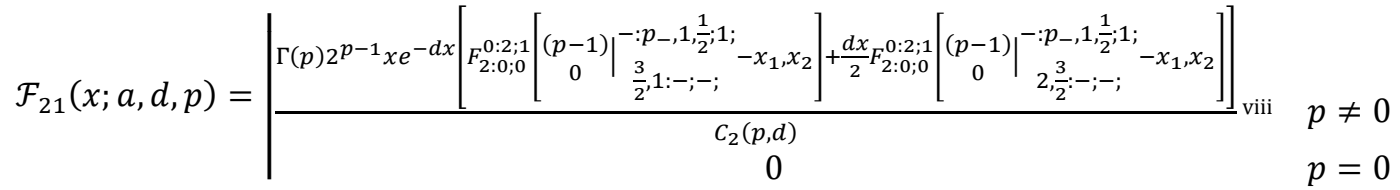

Equation (136) eliminates the singularity at $d=0$ which was present in the left side of (135).

$$
\int_{0}^{x} e^{-t d} t^{2 p+2 k} d t=\frac{\gamma\left(2 p_{1}+2 k, d x\right)}{d^{2} p_{1}+2 k}
$$

The second integral is also pretty straightforward to compute

Substituting (137) into the second summation of (134) yields

$$
\sum_{k=0}^{\infty} \frac{\left[\psi(k+1)+\psi\left(p_{2}+k\right)\right] \int_{0}^{x} e^{-t d} t^{2 p+2 k} d t}{k !(p+k) ! 2^{2 k}}=\sum_{k=0}^{\infty} \frac{\left[\psi(k+1)+\psi\left(p_{2}+k\right)\right] \frac{\gamma\left(2 p_{1}+2 k, d x\right)}{d^{2} p_{1}+2 k}}{2^{2 k} k !(p+k) !}
$$

The recursive implementation algorithm is considered in Appendix D. Since, the direct approach is numerically more accurate and computationally just as efficient as the recursive algorithm, for this particular case the direct approach is

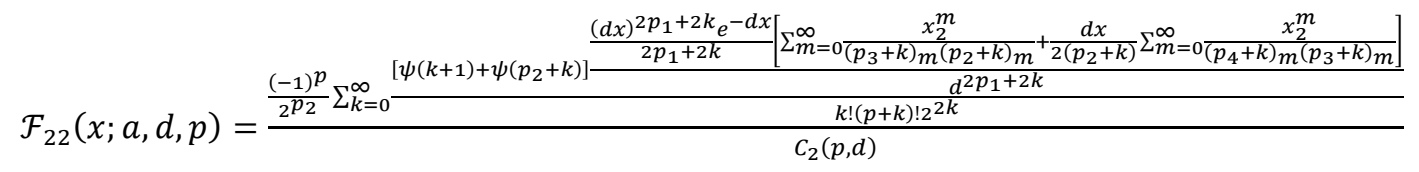


Which is equivalent with

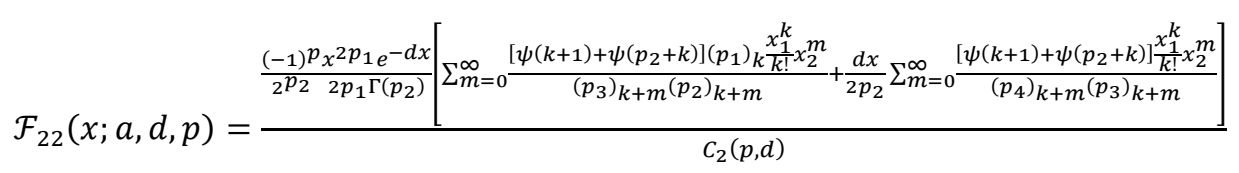

Equation (140) can be written with the help of the modified, generalized Kampé de Fériet function [11]-[13] as follows

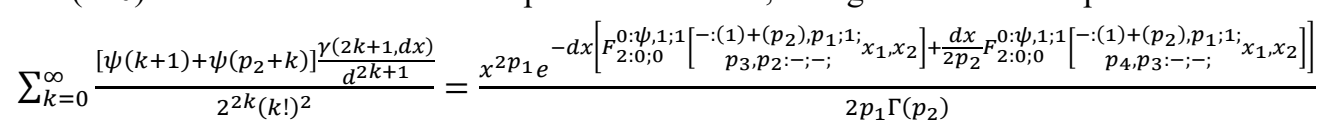

Substituting (141) into (140) produces the final expression of $\mathcal{F}_{22}(x ; a, d, p)$

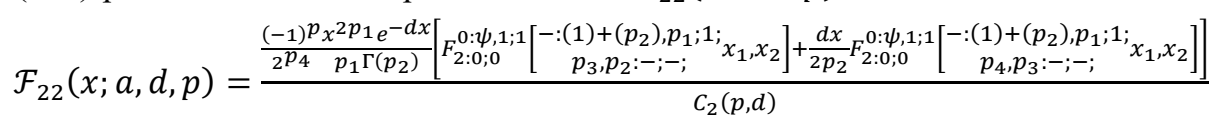

Equation (142) eliminates a singularity for $d=0$, which the recursive approach was not able to; hence, (142) is much better implementation than the recursive algorithm described in Appendix D.

$$
\int_{0}^{x} \ln \frac{t}{2} e^{-t d} t^{2 p+2 k} d t=\left[\left.\ln \left(\frac{t}{2}\right) \frac{\gamma(\alpha, t d)}{d^{\alpha}}\right|_{0} ^{x}=\ln \left(\frac{x}{2}\right) \frac{\gamma(\alpha, d x)}{d^{\alpha}}-\lim _{x \rightarrow 0} \ln \left(\frac{x}{2}\right) \frac{\gamma(\alpha, d x)}{d^{\alpha}}\right]-\int_{0}^{x} \frac{t^{-1} \gamma(\alpha, t d)}{d^{\alpha}} d t
$$

The lower limit of $\ln (x / 2) \gamma(\alpha, d x) / d^{\alpha} \quad k=0,1, \cdots$ can be computed using L'Hôpital's rule (see also (89)) [28]

$$
\lim _{x \rightarrow 0} \ln \left(\frac{x}{2}\right) \frac{\gamma(\alpha, d x)}{d^{\alpha}}=\lim _{x \rightarrow 0} \ln \left(\frac{x}{2}\right) \frac{d^{\alpha} x^{\alpha} e^{-d x} \sum_{n=0}^{\infty} \frac{(1)_{n}(d x)^{n}}{(\alpha+1) n} n !}{\alpha}=\frac{d^{\alpha} \lim _{x \rightarrow 0} \ln \left(\frac{x}{2}\right) x^{\alpha}}{\alpha}=0
$$

Substituting (143), (144) into the second summation of (134) yields

$$
\mathcal{F}_{23}(x ; a, d, p)+\mathcal{F}_{24}(x ; a, d, p)=\frac{\sum_{k=0}^{\infty} \frac{\int_{0}^{x} \ln \frac{t}{2} e^{-t d} t^{2 p+2 k} d t}{2^{2 k} k !(p+k) !}}{C_{2}(p, d)}=\frac{\ln \left(\frac{x}{2}\right) \sum_{k=0}^{\infty} \frac{\frac{\gamma(\alpha, d x)}{d^{\alpha}}}{2^{2 k} k !(p+k) !}-\sum_{k=0}^{\infty} \frac{\int_{0}^{x t^{-1} \gamma(\alpha, t d)} \frac{1}{d^{\alpha}} d t}{2^{2 k} k !(p+k) !}}{C_{2}(p, d)}
$$

Next, we work on the first summation of (145). Substituting Progri 2018 [2], (19) into the second summation yields

$$
\sum_{k=0}^{\infty} \frac{\frac{\gamma\left(2 p_{1}+2 k, d x\right)}{d^{2} p_{1}+2 k}}{2^{2 k} k !(p+k) !}=\frac{e^{-d x}}{2 p_{1} \Gamma\left(p_{2}\right)}\left[\sum_{m=0}^{\infty} \frac{\left(p_{1}\right)_{k} \frac{x_{1}^{k}}{k !} x_{2}^{m}}{\left(p_{3}\right)_{k+m}\left(p_{2}\right)_{k+m}}+\frac{d x}{2 p_{2}} \sum_{m=0}^{\infty} \frac{\frac{x_{1}^{k}}{k !} x_{2}^{m}}{\left(p_{4}\right)_{k+m}\left(p_{3}\right)_{k+m}}\right]
$$

Equation (146) can be written with the help of the Kampé de Fériet function [11]-[13] as follows

$$
\sum_{k=0}^{\infty} \frac{\frac{\gamma\left(2 p_{1}+2 k, d x\right)}{d^{2} p_{1}+2 k}}{2^{2 k} k !(p+k) !}=\frac{x^{2 p_{1}} e^{-d x}}{2 p_{1} \Gamma\left(p_{2}\right)}\left[F_{2: 0 ; 0}^{0: 1 ; 1}\left[\begin{array}{c}
-: p_{1} ; 1 ; \\
p_{3}, p_{2}:-;-;
\end{array} x_{1}, x_{2}\right]+\frac{d x}{2 p_{2}} F_{2: 0 ; 0}^{0: 1 ; 1}\left[\begin{array}{c}
-: p_{1} ; 1 ; \\
p_{4}, p_{3}:-;-;
\end{array} x_{1}, x_{2}\right]\right]
$$

Substituting (147) into the first summation of (145) yields,

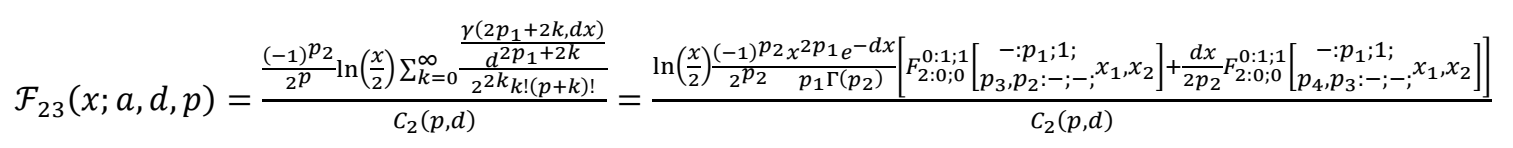

For the computation of the second summation of (150) we employ the series expansion of the incomplete gamma function and after performing the integration we obtain

$$
\mathcal{F}_{24}(x ; a, d, p)=\frac{\frac{(-1) p}{2^{p}} \sum_{k=0}^{\infty} \frac{\sum_{n=0}^{\infty} \frac{(-1)^{n} d^{n} \frac{x^{2 k+2 p_{1}+n}}{\left(2 k+2 p_{1}+n\right)^{2}}}{n !}}{2^{2 k} k !(p+k) !}}{C_{2}(p, d)}=\frac{\frac{(-1)^{p}}{2^{p}} x^{2} p_{1} \sum_{k, n=0}^{\infty} \frac{\frac{x^{2 k}}{2^{2 k}(-d x)^{n}}}{k !} \frac{n !}{\left(2 k+2 p_{1}+n\right)^{2}(p+k) !}}{C_{2}(p, d)}
$$

Next, we perform the expansion into even and odd terms we have 


$$
\mathcal{F}_{24}(x ; a, d, p)=\frac{\frac{(-1)^{p}}{2^{p}} x^{2 p_{1}}\left[\sum_{k, m=0}^{\infty} \frac{\frac{x_{1}^{k}(-d x)^{2 m}}{k !(2 m) !}}{\left(2 k+2 p_{1}+2 m\right)^{2}(p+k) !}+\sum_{k, m=0}^{\infty} \frac{\frac{x_{1}^{k}(-d x)^{2 m+1}}{k !(2 m+1) !}}{\left(2 k+2 p_{2}+2 m\right)^{2}(p+k) !}\right]}{C_{2}(p, d)}
$$

Which is equivalent with

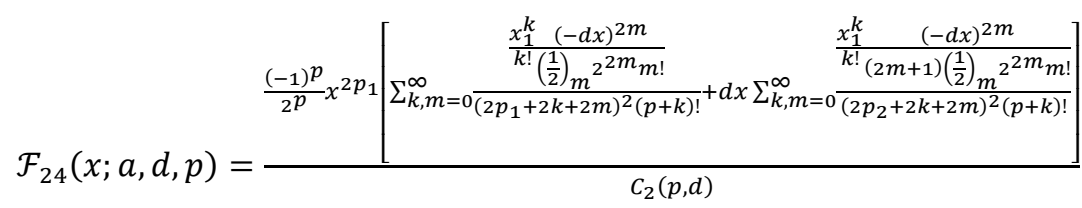

Which is identical to

$$
\mathcal{F}_{24}(x ; a, d, p)=\frac{\frac{(-1)^{p}}{2^{p}} x^{2 p_{1}}\left[\frac{\frac{x_{1}^{k} x_{2}^{m}}{k !}\left(\frac{1}{2}\right)_{m}^{m !}}{\frac{1}{4} \sum_{k, m=0}^{\infty} \frac{d x}{\left(p_{1}+k+m\right)^{2}(p+k) !}-\frac{x_{1}^{k}}{8} \sum_{k, m=0}^{\infty} \frac{x_{2}^{m}}{k !\left(m+\frac{1}{2}\right)\left(\frac{1}{2}\right)_{m}^{m !}}\left(p_{2}+k+m\right)^{2}(p+k) !}\right]}{C_{2}(p, d)}
$$

Equation (152) can be written as follows

$$
\mathcal{F}_{24}(x ; a, d, p)=\frac{\frac{(-1) p_{x^{2} p_{1}}}{2^{p} \Gamma\left(p_{2}\right)}\left[\frac{1}{4 p_{1} p_{1}} \sum_{k, m=0}^{\infty} \frac{\left(p_{1}\right)_{k+m}\left(p_{1}\right)_{k+m} \frac{x_{1}^{k} x_{2}^{m}}{k ! m !}}{\left(p_{3}\right)_{k+m}\left(p_{3}\right)_{k+m}\left(p_{2}\right)_{k}\left(\frac{1}{2}\right)_{m}}-\frac{d x}{4 p_{2} p_{2}} \sum_{k, m=0}^{\infty} \frac{\left(p_{2}\right)_{k+m}\left(p_{2}\right)_{k+m} \frac{x_{1}^{k} x_{2}^{m}}{k ! m !}}{\left(p_{4}\right)_{k+m}\left(p_{4}\right)_{k+m}\left(p_{2}\right)_{k}\left(\frac{3}{2}\right)_{m}}\right]}{C_{2}(p, d)}
$$

Finally, Dr. Progri has successfully derived for the first time Kampé de Fériet function [11]-[13] as follows that this summation which can be written with the help of the

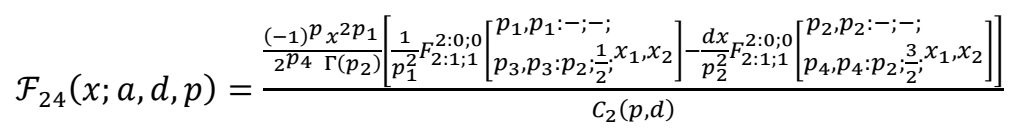

Finally, substituting (136), (142), (148) and (154) into (136) $\quad \mathcal{F}_{\mathrm{GBessel} 2}(x ; a, d, p)$ produces the desired closed form expression of

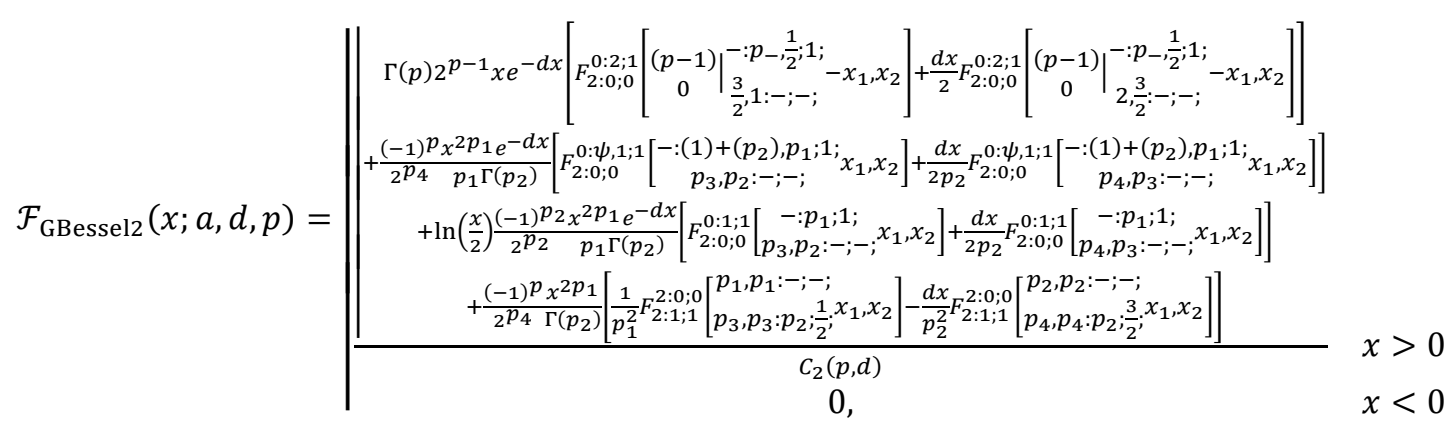

Equation (155) is an original expression not published anywhere else; however, care must be shown in the computation of the Kampé de Fériet function [2].

The obvious question is as follows. Is (155) the right equation? While numerically we will test this at the numerical,

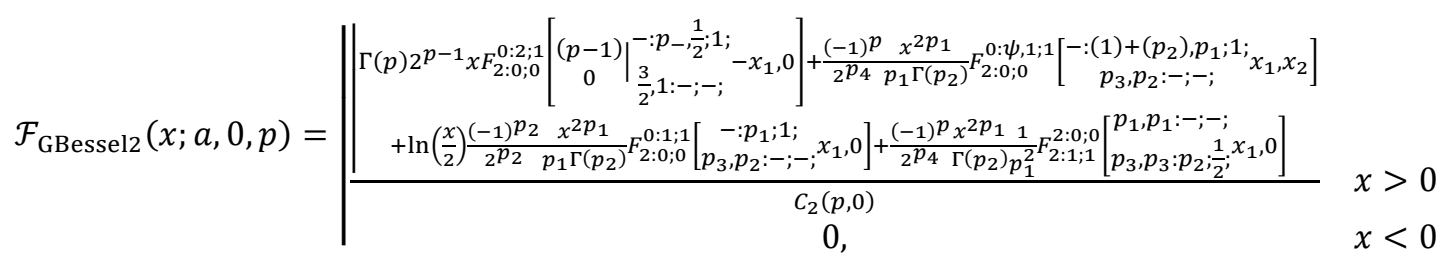


Evaluating the four Kampé de Fériet functions [11]-[13] at $\quad d=0$ yields

$$
\begin{aligned}
& F_{2: 0 ; 0}^{0: 2 ; 1}\left[\begin{array}{c|c}
(p-1) & \begin{array}{c}
-: p_{-}, \frac{1}{2} ; 1 ; \\
0
\end{array} \mid x_{1}, 0 \\
\frac{3}{2}, 1:-;-;
\end{array}\right]=\sum_{k, m=0}^{p-1, \infty} \frac{(p)_{-k}(1)_{k}\left(\frac{1}{2}\right)_{k}(1)_{m} \frac{\left(-x_{1}\right)^{k} 0^{m}}{k !} m !}{\left(\frac{3}{2}\right)_{k+m}(1)_{k+m}}=\sum_{k=0}^{p-1} \frac{(p)_{-k}\left(\frac{1}{2}\right)_{k} \frac{\left(-x_{1}\right)^{k}}{k !}}{\left(\frac{3}{2}\right)_{k}} \\
& F_{2: 0 ; 0}^{0: \psi, 1 ; 1}\left[\begin{array}{c}
-:(1)+\left(p_{2}\right), p_{1} ; 1 ; \\
p_{3}, p_{2}:-;-;
\end{array} x_{1}, x_{2}\right]=\sum_{k=0}^{\infty} \frac{\left[\psi(1+k)+\psi\left(p_{2}+k\right)\right]\left(p_{1}\right)_{k} \frac{x_{1}^{k}}{k !}}{\left(p_{3}\right)_{k}\left(p_{2}\right)_{k}}={ }_{\psi, 1} F_{2}\left(\left[(1)+\left(p_{2}\right), p_{1}\right] ; p_{3}, p_{2} ; x_{1}\right)
\end{aligned}
$$

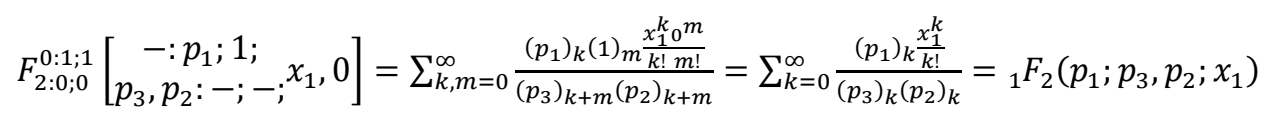

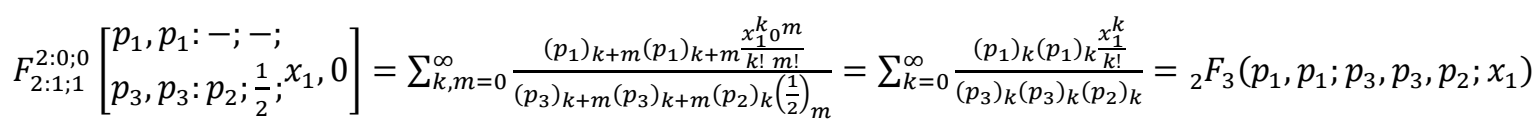

Substituting (157)-(160) into (158) yields

$$
\mathcal{F}_{\mathrm{GBessel} 2}(x ; a, 0, p)=\frac{\begin{array}{l}
\Gamma(p) 2^{p-1} x \sum_{k=0}^{p-1} \frac{(p)-k\left(\frac{1}{2}\right)_{k} \frac{\left(-x_{1}\right)^{k}}{k !}}{\left(\frac{3}{2}\right)_{k}}+\frac{(-1)^{p_{2} p_{1}}}{2^{p_{4} p_{1} \Gamma\left(p_{2}\right)} \psi, 1} F_{2}\left[(1)+\left(p_{2}\right), p_{1} ; p_{3}, p_{2} ; x_{1}\right] \\
+\ln \left(\frac{x}{2}\right) \frac{(-1) p_{2} x^{2} p_{1}}{2^{p_{2}} p_{1} \Gamma\left(p_{2}\right)^{1}} F_{2}\left(p_{1} ; p_{3}, p_{2} ; x_{1}\right)+\frac{(-1) p_{x^{2} p_{1}}}{2^{p_{4}} \Gamma\left(p_{2}\right) p_{1}^{2}{ }^{2} F_{3}\left(p_{1}, p_{1} ; p_{3}, p_{3}, p_{2} ; x_{1}\right)}
\end{array}}{C_{2}(p, 0)}
$$

Equation (161) produces exactly the same answer as (103); thus, completing the proof for $d=0, p \neq 0$.

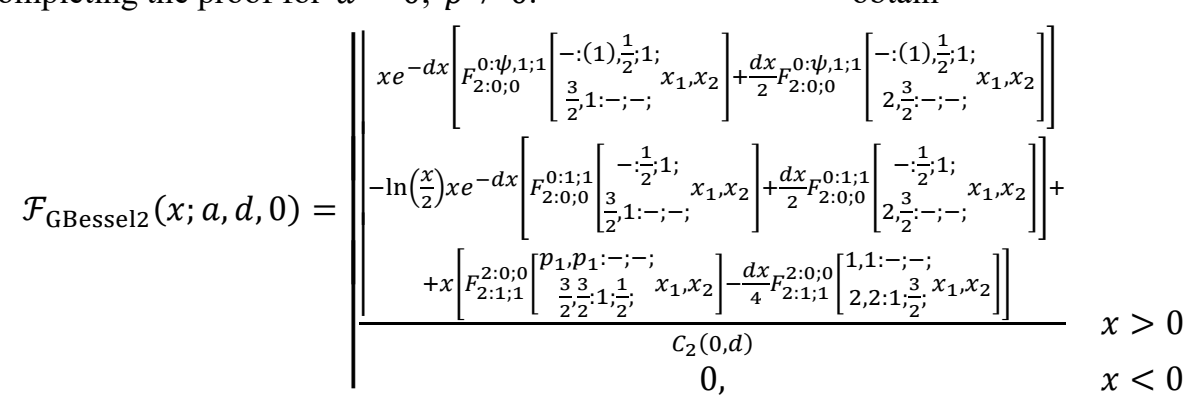

Equation (162) is identical to (127); thus, completing the proof for $d \neq 0, p=0$.

Finally, we consider the case for $d=0, p=0$ from (161),

$$
\mathcal{F}_{\mathrm{GBessel} 2}(x ; a, 0,0)=\mid \begin{array}{cc}
\frac{x\left[\psi, 1 F_{2}\left((1), \frac{1}{2} ; \frac{3}{2}, 1 ; x_{1}\right)-\ln \left(\frac{x}{2}\right){ }_{1} F_{2}\left(\frac{1}{2} ; \frac{3}{2}, 1 ; x_{1}\right)+{ }_{2} F_{3}\left(\frac{1}{2}, \frac{1}{2} ; \frac{3}{2}, \frac{3}{2}, 1 ; x_{1}\right)\right]}{C_{2}(0,0) \equiv \pi} & x>0 \\
0 & x \leq 0
\end{array}
$$

Equation (163) is identical to (98); thus, completing the proof for $d=0, p=0$.

This concludes the derivations of the special case for $d \neq 0$ and $p \neq 0, p$ a positive integer.

Next we consider the numerical, theoretical results.

\section{Numerical, Theoretical Results}

Progri 2016 [1] numerical, theoretical results section contains the first set of simulation results of pdfs and approximated numerical cdfs of the generalized Bessel function distributions of the first and second kinds based analytical derivations presented in Progri 2016 [1]; i.e., in Progri 2016, we did not provide any simulation results of the exact closed form expression of the cdfs of the generalized Bessel function distributions of the first and second kinds.

Hence, in this section we present for the first time the MATLAB simulation results of the of the exact closed form expression of the cdfs of the generalized Bessel function distributions of the first and second kinds based on closed form expressions (117) and (142) in Progri 2016 and (25) in the current journal paper.

Moreover, we want to ensure the reader that when we produced the simulation results of this section every single equation of Progri 2016 and of the current journal paper has 
been tested; hence, the simulation results go far beyond the simulation results of just closed form expressions (117) and (142) in Progri 2016 and (25) in the current journal paper.

\subsection{Special Case: $d=0, p \neq 0$ not an Integer}

In this subsection we present, numerical results of the generalized Bessel function of the first and second kinds using the exact closed form expression found in this paper and in Progri 2016 [1].

Figure 1(a) shows the plots of (top) the generalized Bessel of the second kind pdf and cdf for $a=2, d=0$, and $p=0.5$ for the integral and linear numerical approximation; (bottom) cdf using (62) in the current journal paper and (142) from Progri 2016 [1].

Figure 1(b) shows absolute errors of the generalized Bessel of the first kind cdf for $a=2, d=0, p=0.5$.

Absolute errors of the generalized Bessel function distribution of the second kinds are shown in Figs. 1(b) and 2(b).

Figure 2 (a) shows the plots of (top) the generalized Bessel of the second kind pdf and cdf for $a=2, d=0$, and $p=$ -0.25 for the integral and linear numerical approximation; (bottom) cdf using (62) in the current journal paper and (142) from Progri 2016 [1].

Figure 2 (b) shows absolute errors of the generalized Bessel of the second kind cdf for $a=2, d=0, p=-0.25$; i.e., $p$ not an integer.

The important results here are that even for non-integer values of $p$ the generalized Bessel of the first and second kinds pdfs and cdfs are validated. For this special case the MATLAB integral function provides an erroneous solution due to the presence of infinity or singularity.

\subsection{Special Case: $\boldsymbol{d}=\mathbf{0}, \boldsymbol{p}=\mathbf{0}$}

The pdf and cdfs and the absolute errors of the generalized Bessel function distribution of the second kinds corresponding to the special case of $d=0$, and $p=0$ are shown in Fig. 3 .

Figure 3 (a) shows the plots of (top) the generalized Bessel of the second kind pdf and cdf for $a=2, d=0$, and $p=0$ for the integral and linear numerical approximation; (bottom) cdfs using (98) and (103) in the current journal paper.

Figure 3 (b) shows absolute errors of the generalized Bessel of the second kind cdf for $a=2, d=0, p=0$; i.e., $p$ an integer.

For this special case the cdfs computed from using (98) and (103) are fourteen to sixteen orders of magnitude as accurate as the MATLAB integral function.

\subsection{Special Case: $d=0, p$ a Positive Integer}

The pdf and cdfs and the absolute errors of the generalized Bessel function distribution of the second kinds corresponding to the special case of $d=0$, and $p$ a positive integer are shown in Fig. 4.

Figure 4 (a) shows the plots of (top) the generalized Bessel of the second kind pdf and cdf for $a=2, d=0$, and $p=2$ for the integral and linear numerical approximation; (bottom) cdfs using (103) Option 1 and (187) Option 2 in the current journal paper.

Figure 4 (b) shows absolute errors of the generalized Bessel of the second kind cdf for $a=2, d=0, p=2$; i.e., $p$ an integer.

For this special case the cdfs computed from using (103) and (187) are fourteen to sixteen orders of magnitude as accurate as the MATLAB integral function.

\subsection{Special Case: $d \neq 0, p=0$}

The pdf and cdfs and the absolute errors of the generalized Bessel function distribution of the second kinds corresponding to the special case of $d=0.5$, and $p=0$ shown in Fig. 5 .

Figure 5 (a) shows the plots of (top) the generalized Bessel of the second kind pdf and cdf for $a=2, d=0.5$, and $p=0$ for the integral and linear numerical approximation; (bottom) cdfs using (127) Option 2 and (155) Option 2 in the current journal paper.

Figure 5 (b) shows absolute errors of the generalized Bessel of the second kind cdf for $a=2, d=0.5, p=0$; i.e., $p$ an integer.

For this special case the cdfs computed from using (127) and (155) are fourteen to sixteen orders of magnitude as accurate as the MATLAB integral function.

\subsection{Special Case: $d \neq 0, p$ a Positive Integer}

The pdf and cdfs and the absolute errors of the generalized Bessel function distribution of the second kinds corresponding to the special case of $d \neq 0$, and $p=0$ a positive integer are shown in Fig. 6.

Figure 6 (a) shows the plots of (top) the generalized Bessel of the second kind pdf and cdf for $a=2, d=0.5$, and $p=2$ for the integral and linear numerical approximation; (bottom) cdfs using (155) Option 2 and (155) Option 3 in the current journal paper. 
TABLE I: COMPUTATION TIME OF CDFS

\begin{tabular}{lccccc}
\hline \hline No & $\begin{array}{c}\tau_{\text {int }} \\
(\mathrm{s})\end{array}$ & $\begin{array}{c}\tau_{\text {lap }} \\
(\mathrm{ms})\end{array}$ & $\begin{array}{c}\tau_{c f 1} \\
(\mathrm{~s})\end{array}$ & $\begin{array}{c}\tau_{c f 2} \\
(\mathrm{~s})\end{array}$ & $\begin{array}{c}\tau_{\Sigma} \\
(\mathrm{s})\end{array}$ \\
\hline \hline $1 \mathrm{a}$ & 4.15 & 1.2 & 0.005 & 0.013 & 4.17 \\
$1 \mathrm{~b}$ & 4.16 & 3.1 & 0.014 & 0.029 & 4.20 \\
2 & 0.13 & 0.1 & 0.004 & 0.01 & 0.14 \\
3 & 0.16 & 3.5 & 0.008 & 0.012 & 0.18 \\
4 & 4.14 & 0.1 & 0.22 & 0.13 & 4.54 \\
5 & 4.29 & 0.01 & 0.31 & 0.17 & 4.62 \\
\hline
\end{tabular}

TABLE II: COMPUTATION TIME OF CDF (155) VIA OPTIONS 1-4

\begin{tabular}{cccccc}
\hline \hline $\mathrm{N}$ & $\begin{array}{c}\tau_{\text {int }} \\
(\mathrm{s})\end{array}$ & $\begin{array}{c}\tau_{\text {lap }} \\
(\mathrm{ms})\end{array}$ & $\begin{array}{c}\tau_{c f 1} \\
(\mathrm{~s})\end{array}$ & $\begin{array}{c}\tau_{c f 2} \\
(\mathrm{~s})\end{array}$ & $\begin{array}{c}\tau_{\Sigma} \\
(\mathrm{s})\end{array}$ \\
\hline \hline 15 & 4.2 & 3.1 & $0.35(1)$ & $1.56(2)$ & 6.12 \\
25 & 4.29 & 0.6 & $0.50(1)$ & $1.66(2)$ & 6.45 \\
50 & 4.19 & 3.2 & $1.21^{\mathrm{ix}}(1)$ & $2.00(2)$ & 7.41 \\
15 & 4.18 & 0.1 & $38.26(3)$ & $38.14(4)$ & 80.58 \\
25 & 4.21 & 0.1 & $60.08(3)$ & $60.42(4)$ & 124.71 \\
50 & 4.23 & 0.032 & $116.12(3)$ & $115.89(4)$ & 236.24 \\
\hline
\end{tabular}

TABLE III: SAME AS II USING PROGRI's IMPLEMENTATION

\begin{tabular}{cccccc}
\hline \hline $\mathrm{N}$ & $\begin{array}{c}\tau_{\text {int }} \\
(\mathrm{s})\end{array}$ & $\begin{array}{c}\tau_{\text {lap }} \\
(\mathrm{ms})\end{array}$ & $\begin{array}{c}\tau_{c f 1} \\
(\mathrm{~s})\end{array}$ & $\begin{array}{c}\tau_{c f 2} \\
(\mathrm{~s})\end{array}$ & $\begin{array}{c}\tau_{\Sigma} \\
(\mathrm{s})\end{array}$ \\
\hline \hline 15 & 4.2 & 3.1 & $0.27(1)$ & $0.24(2)$ & 4.69 \\
25 & 4.29 & 0.6 & $0.45(1)$ & $0.32(2)$ & 4.92 \\
50 & 4.14 & 3.2 & $1.21^{\mathrm{x}}(1)$ & $0.74(2)$ & 6.08 \\
15 & 4.18 & 0.1 & $0.18(3)$ & $0.16(4)$ & 4.50 \\
25 & 4.21 & 0.1 & $0.20(3)$ & $0.17(4)$ & 4.52 \\
50 & 4.23 & 0.032 & $0.25(3)$ & $0.22(4)$ & 4.61 \\
\hline
\end{tabular}

Figure 6 (b) shows absolute errors of the generalized Bessel of the second kind cdf for $a=2, d=0.5, p=2$; i.e., $p$ an integer.

For this special case the cdfs computed from using (127) and (155) are fourteen to sixteen orders of magnitude as accurate as the MATLAB integral function.

Figures 1-6 are necessary because they show that the cdf accuracy performance is outstanding; hence, it remains to test the computational speed performance.

The computational times, performed in an AMD A6-3420 APU with Radeon ${ }^{\mathrm{TM}}$ HD Graphics $1.5 \mathrm{GHz}$ processor 64-bit operating system, of all the cdfs is considered in Tab. I. In the first row of Tab. I the computation times of special case discussed in Fig. 1 are reported. And so forth.
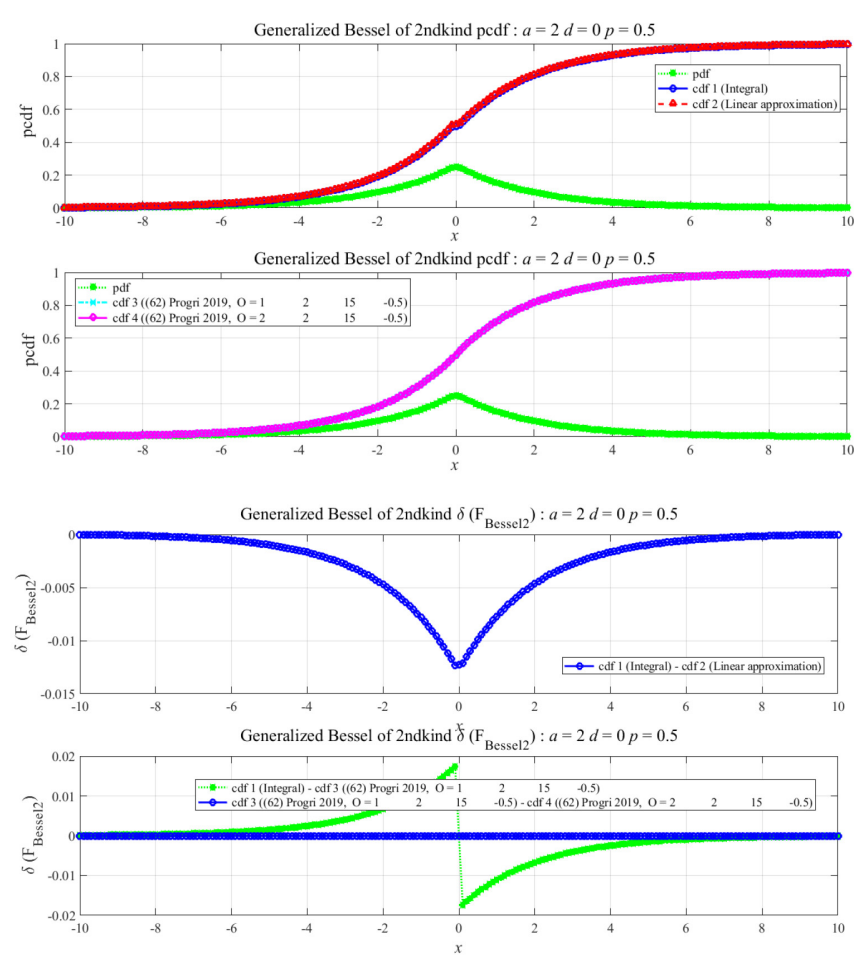

(a) (top) original generalized Bessel of the second kind pdf and cdf for $a=2, d=0$, and $p=0.5$, using the integral and linear numerical approximation; (bottom) original generalized Bessel of the second kind pdf and cdf for $a=12, d=0$, and $p=0.5$ for (62) and (142) from Progri 2016 [1] Option 3 of the Kampé de Fériet function.

(b) (top) absolute error of the original generalized Bessel of the second kind pdf and cdf for $a=2, d=0$, and $p=0.5$, integral - linear numerical approximation; (bottom) absolute error of the original generalized Bessel of the second kind pdf and cdf for $a=2, d=0$, and $p=0.5$, for (62) - (142) from Progri 2016 [1] Option 3 of the Kampé de Fériet function; $p$ not an integer.

FIGURE 1: Numerical calculations of the generalized Bessel of the (a) second and (b) second kind absolute error pdf and cdf for (62) and (142) from Progri 2016 [1] Option 3 of the Kampé de Fériet function for $a=2, d=0$, and $p=0.5$.

There are six columns in Tab. I: The first column corresponds to the number of rows; the second column corresponds to the MATLAB integral function computations, the third column corresponds to the linear approximation, the fourth to the first closed form expression discussed in Sect. 3, the fifth to the total computation time. Based on the computation times reported in Tab. I, 6/6 times, the computation times of the cdfs based on the closed form expression provided in Sect. 3 is significantly (9$26 \times$ ) faster than the MATLAB integral function. 

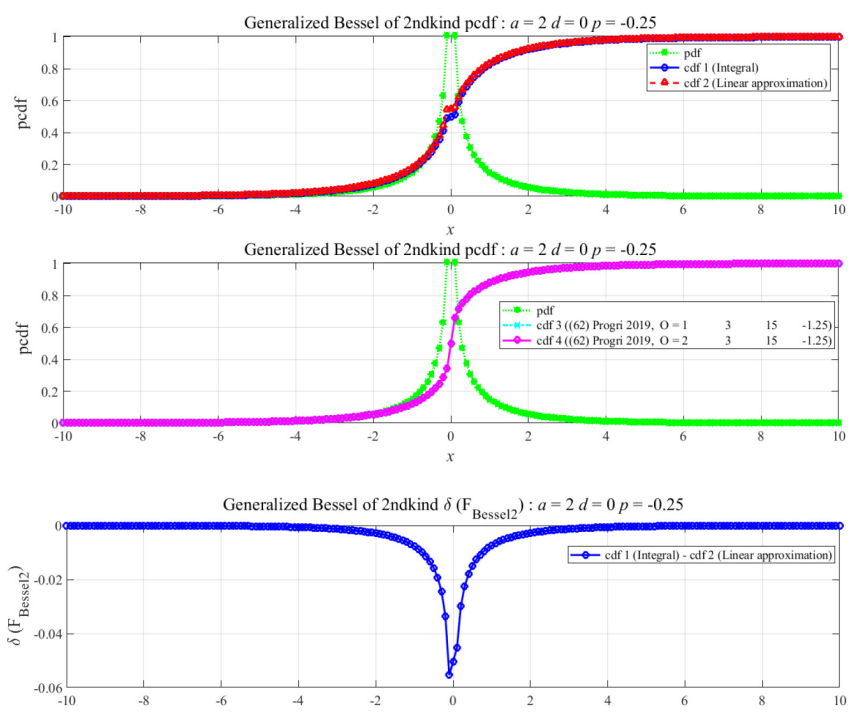

Generalized Bessel of 2ndkind $\delta^{x}\left(\mathrm{~F}_{\text {Bessel2 }}\right): a=2 d=0 p=-0.25$

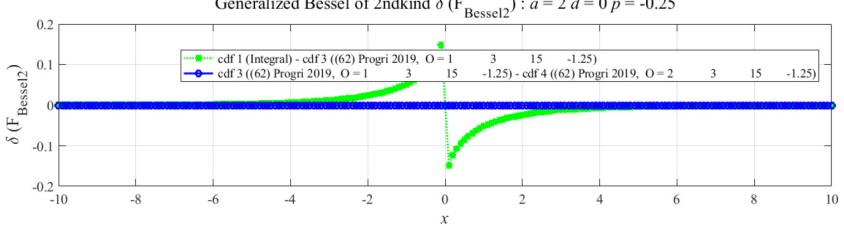

(a) (top) original generalized Bessel of the second kind pdf $f^{\mathrm{xi}}$ and cdf for $a=2, d=0$, and $p=-0.25$, for the integral ${ }^{\mathrm{xii}}$ and linear numerical approximation; (bottom) original generalized Bessel of the second kind pdf and cdf for $a=12$, $d=0$, and $p=-0.25$ for (62) and (142) from Progri 2016 [1] Option 3 of the Kampé de Fériet function.

(b) (top) absolute error of the original generalized Bessel of the second kind pdf and cdf for $a=2, d=0$, and $p=-0.25$, integral - linear numerical approximation; (bottom) absolute error of the original generalized Bessel of the second kind pdf and cdf for $a=2, d=0$, and $p=-0.25$, for (62) - (142) from Progri 2016 [1] Option 3 of the Kampé de Fériet function; $p$ not an integer.

FIGURE 2: Numerical calculations of the generalized Bessel of the (a) second and (b) second kind absolute error pdf and cdf for (62) Option 2 of the Kampé de Fériet function and (142) from Progri 2016 [1] Option 3 of the Kampé de Fériet function for $a=2, d=0$, and $p=-0.25 ; p$ not an integer.

For example. If we consider 15 terms during the computation of the cdf via (155) (see Tab. II) then: (1) the integral approach is 42 thousand times less efficient than the linear approximation; (2) The implementation of the the Kampé de Fériet function via options 1 and 2 is 12/2.5 times more efficient than the direct integral via MATLAB; (3) The implementation of the Kampé de Fériet function using the MATLAB hypergeom function via options 3 and 4 is 9 times less efficient than the direct integral via MATLAB.
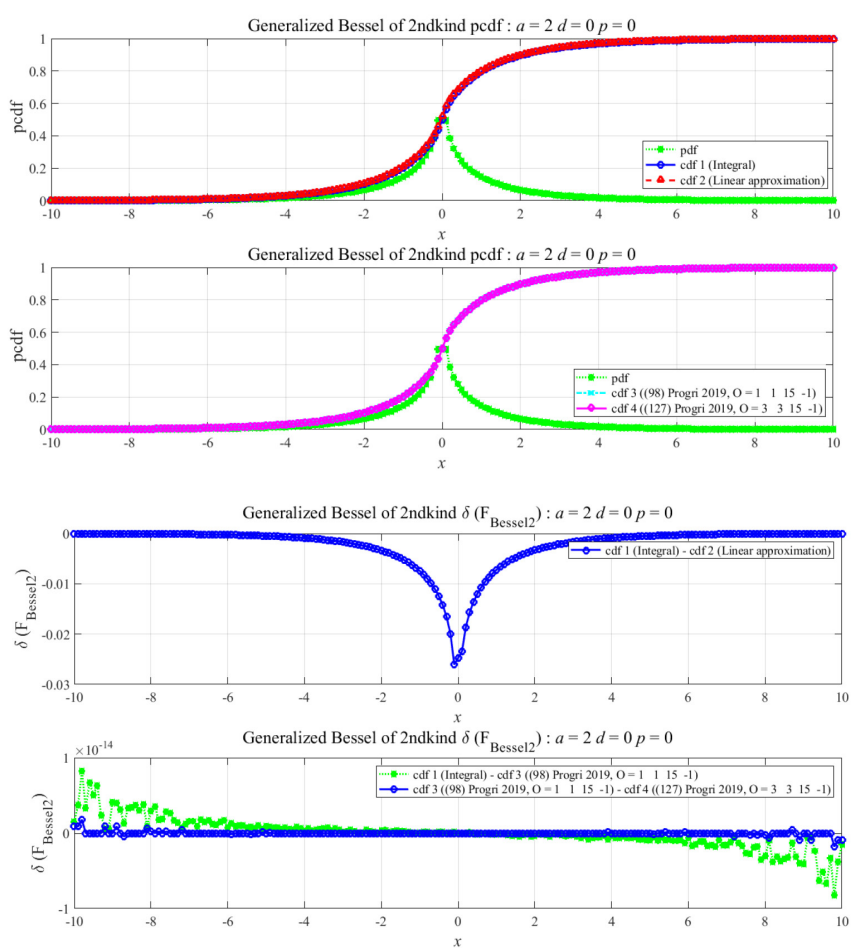

(a) (top) original generalized Bessel of the second kind pdf ${ }^{x i i}$ and cdf for $a=2, d=0$, and $p=0$, for the integral ${ }^{\mathrm{xiv}}$ and linear numerical approximation; (bottom) original generalized Bessel of the second kind pdf and cdf for $a=12, d=0$, and $p=0$ for (98) Option 1 of the Kampé de Fériet function and (127) ${ }^{\mathrm{xv}}$ Option 3 of the Kampé de Fériet function; $p$ an integer.

(b) (top) absolute error of the original generalized Bessel of the second kind pdf and cdf for $a=2, d=0$, and $p=0$, integral - linear numerical approximation; (bottom) absolute error of the original generalized Bessel of the second kind pdf and cdf for $a=2, d=0$, and $p=0$, for (98) Option 1 of the Kampé de Fériet function - (127) Option 3 of the Kampé de Fériet function; $p$ an integer.

FIGURE 3: Numerical calculations of the generalized Bessel of the (a) second and (b) second kind absolute error pdf and cdf for (98) Option 1 of the Kampé de Fériet function and (127) Option 3 of the Kampé de Fériet function for $a=2, d=0$, and $p=0 ; p$ an integer.

The implementation of the Kampé de Fériet function using the Progri's phypergeom function via options 3 and 4 in Tab. III is 527 times more efficient than the save via MATLAB hypergeom function. In summary, the Progri implementation of the Kampé de Fériet function (see Progri 2018 [2], Appendix A) is the optimum in performance and speed (option 4); but it requires Progri implementation of the generalized hypergeometric function. 

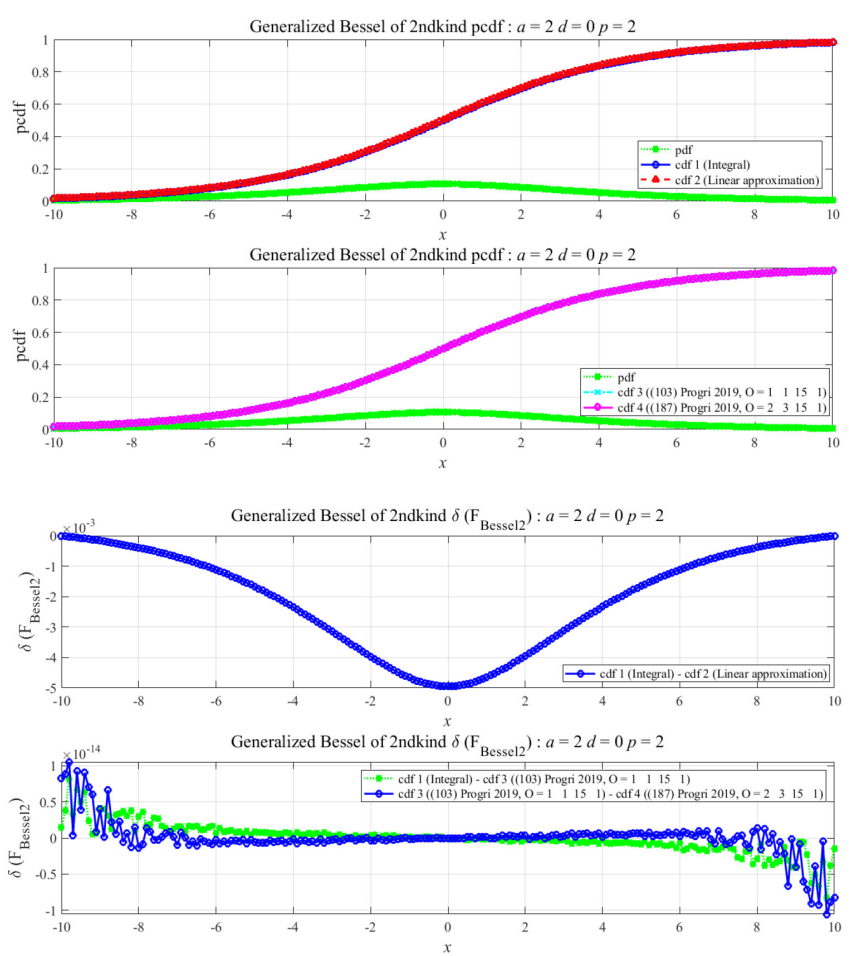

(a) (top) original generalized Bessel of the second kind pdf and cdf for $a=2, d=0$, and $p=2$, for the integral and linear numerical approximation; (bottom) original generalized Bessel of the second kind pdf and cdf for $a=2, d=0$, and $p=2$ for (103) Option 1 or the recursive algorithm and (187) Option 3 of the Kampé de Fériet function; $p$ an integer.

(b) (top) absolute error of the original generalized Bessel of the second kind pdf and cdf for $a=2, d=0$, and $p=3$, integral - linear numerical approximation; (bottom) absolute error of the original generalized Bessel of the second kind pdf and cdf for $a=2, d=0$, and $p=2$, for (103) Option 1 or the recursive algorithm - (187) Option 3 of the Kampé de Fériet function; $p$ an integer.

FIGURE 4: Numerical calculations of the generalized Bessel of the (a) second and (b) second kind absolute error pdf and cdf for (103) Option 1 or the recursive algorithm and (187) Option 3 of the Kampé de Fériet function for $a=2, d=0$, and $p=$ $2 ; p$ an integer.

\section{Conclusions}

The main focus on this masterpiece journal paper is to validate the entire derivations of the closed form expressions provided in Sects. 2 and 3 namely: (1) the cdf of the generalized Bessel of the second kind pdfs/cdfs in the current journal paper corresponding to five special cases: one for non-integer values of $p$ and four for integer values of $p$.
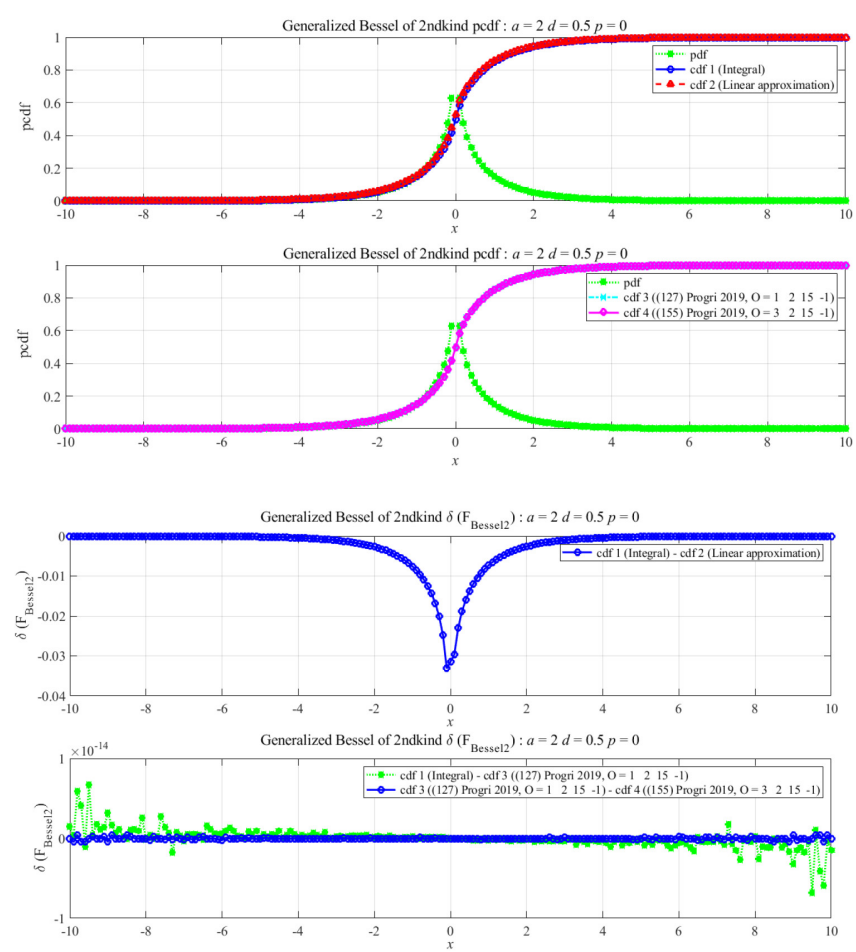

(a) (top) original generalized Bessel of the second kind pdf $\mathrm{fvi}^{\mathrm{xi}}$ and cdf for $a=2, d=0.5$, and $p=0$, for the integral ${ }^{\mathrm{xvii}}$ and linear numerical approximation; (bottom) original generalized Bessel of the second kind pdf and cdf for $a=2, d=0.5$, and $p=0$ for (127) Option 2 of the Kampé de Fériet function and (155) Option 2 of the Kampé de Fériet function; $p=0$ an integer.

(b) (top) absolute error of the original generalized Bessel of the second kind pdf and cdf for $a=2, d=0.5$, and $p=0$, integral - linear numerical approximation; (bottom) absolute error of the original generalized Bessel of the second kind pdf and cdf for $a=2, d=0.5$, and $p=0$, for (127) Option 2 of the Kampé de Fériet function - (155) Option 2 of the Kampé de Fériet function; $p$ an integer.

FIGURE 5: Numerical calculations of the generalized Bessel of the (a) second and (b) second kind absolute error pdf and cdf for (127) Option 2 of the Kampé de Fériet function and (155) Option 2 of the Kampé de Fériet function for $a=2, d=0.5$, and $p=0 ; p$ an integer.

Option 1 does not produce any output when the number of terms exceeds fifty. Option 2 is the most accurate and the most computationally stable option for the implementation of the Kampé de Fériet function. Option 2 was added for the first time to perform the computations of this journal paper.

For cases discussed in Figs. 1 and 2, the direct integration is not accurate due to the presence of infinity or singularity. 

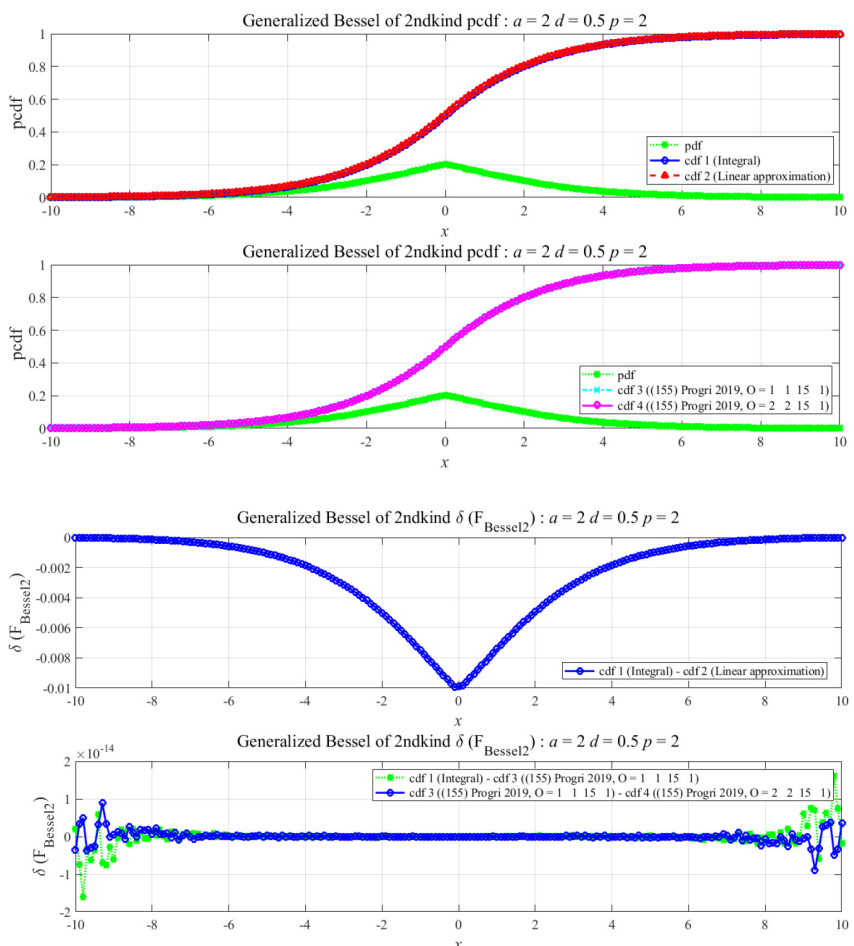

(a) (top) original generalized Bessel of the second kind pdf and cdf for $a=2, d=0.5$, and $p=2$, for the integral and linear numerical approximation; (bottom) original generalized Bessel of the second kind pdf and cdf for $a=2, d=0.5$, and $p=2$ for (155) Option 1 of the Kampé de Fériet function and (155) Option 2 of the Kampé de Fériet function; $p$ an integer.

(b) (top) absolute error of the original generalized Bessel of the second kind pdf and cdf for $a=2, d=0.5$, and $p=2$, integral - linear numerical approximation; (bottom) absolute error of the original generalized Bessel of the second kind pdf and cdf for $a=2, d=0.5$, and $p=2$, for (155) Option 1 of the Kampé de Fériet function - (155) Option 2 of the Kampé de Fériet function; $p$ an integer.

FIGURE 6: Numerical calculations of the generalized Bessel of the (a) second and (b) second kind absolute error pdf and cdf for (155) Option 1 of the Kampé de Fériet function and (155) Option 2 of the Kampé de Fériet function for $a=2, d=0.5$, and $p=2 ; p$ an integer.

We can say with absolute certainty that all the closed form expressions provided in Progri 2016 [1], 2018 [2] have been simulated and tested. The numerical results obtained from the closed form expressions in Fig. 1 to 6 are several orders of magnitude more accurate than those obtained from the linear approximation simulation results presented initially in Progri $2016[1]$.

Numerical results are derived for each case to validate the theoretical models presented in the paper.
For each generalized Bessel distribution model the closed form expression of cdf is given by means of series expansion of the modified Bessel functions which leads to incomplete gamma functions, hypergeometric series, and the Kampé de Fériet functions.

It is also shown that for most practical applications the generalized Bessel function distribution cdf of the first and second kinds can be represented in closed form by means of the family of well-known the Kampé de Fériet functions to within fourteen to fifteen orders of magnitude accuracy.

In summary, the Progri implementation of the generalized hypergeometric function and the Kampé de Fériet function is the optimum in performance and speed.

\section{Acknowledgement}

This work was supported by Giftet Inc. executive office.

I want to profoundly thank the MathWorks at Natick, Massachusetts for providing a sponsored MATLAB licence to Giftet Inc. part of the Indoor Geolocation Systems MATLAB Library development that will enable the results of this work to be published in Dr. Progri pioneer publication Indoor Geolocation Systems - Theory and Applications. Vol. I (Not yet available in print) [17].

This journal paper is dedicated to four special men in my life: my grandfather, Xhevdet Progri, my dear father, Fiqiri Progri, my father's first cousin Dr. Peter Demir, and the $41^{\text {st }}$ President of the United States of America, George H.W. Bush.

\section{$7 \quad$ References}

[1] I. Progri, "Generalized Bessel function distributions," $J$. Geol. Geoinfo. Geointel., vol. 2016, article ID 2016071602, 15 pg., Nov. 2016. DOI: http://doi.org/10.18610/JG3.2016.071602, http://giftet.com/JG3/2016/071602.pdf.

[2] I. Progri, "Efficient computation of generalized Bessel function distributions," J. Geol. Geoinfo. Geointel., vol. 2018, article ID 2016071605, 12 pg., Nov. 2018. DOI: http://doi.org/10.18610/JG3.2018.071605, http://giftet.com/JG3/2018/071605.pdf.

[3] I. Progri, "Exponential generalized Beta distribution," $J$. Geol. Geoinfo. Geointel., vol. 2016, article ID 2016071603, 18 pg., Nov. 2016. DOI: 
http://doi.org/10.18610/JG3.2016.071603, http://giftet.com/JG3/2016/071603.pdf.

[4] I. Progri, "Hypergeometric function partial derivatives," vol. 2016, article ID 2016071604, 21 pg., Nov. 2016. DOI: http://doi.org/10.18610/JG3.2016.071604, http://giftet.com/JG3/2016/071604.pdf.

[5] I.F. Progri, P. Huang, Y. Pi, X. Xia, "Indoor adaptive GNSS signal acquisition. Part 1: theory and simulations," J. Geol. Geoinfo. Geointel., vol. 2016, article ID 2016071601, 19 pg., Nov. 2016. DOI: http://doi.org/10.18610/JG3.2016.071601, http://giftet.com/JG3/2016/071601.pdf.

[6] 1. Progri, I., Huang, P., Pi, Y., Xia, X., "Indoor adaptive GNSS signal acquisition - theory and simulations," in Proc. ION-ITM, Monterey, CA, pp. 902-931, Jan. 2016.

[7] I. Progri, "Advanced anti-jam indoor adaptive GNSS signal acquisition: part 1, normal distribution - theory," in Proc. ION-ITM, Monterey, CA, pp. 1390-1419, Jan. 2017.

[8] I. Progri, "Maximum likelihood GNSS parameter estimation: part 2, Bessel distribution:-Theory and simulation," in Proc. ION-ITM, Reston, VA, pp. 589-610, Jan. 29-Feb. 1, 2018.

[9] I. Progri, "Advanced anti-jam indoor adaptive GNSS signal acquisition: part 2, Bessel distribution-Theory," in Proc. ION-ITM, Reston, VA, pp. 440-466, Jan. 29-Feb. 1, 2018.

[10]Anon, "Kampé de Fériet function," Wikipedia, the free encyclopedia,

Dec.

2015 , https://en.wikipedia.org/wiki/Kamp\%C3\%A9_de_F\%C3 $\%$ A9riet_function.

[11]P. Appell, J., Kampé de Fériet, Fonctions Hypergéométriques et Hypersphériques; Polynomes d'Hermites. Paris: Gauthier-Villars, 1926.

[12] S. Ali, "On a reducibility of the Kampé de Fériet function," Adv. Info. Techn. Manag., vol. 1, no. 1, pp. 24-27, Mar. 2012.

[13] S. Ali, “A transformation formula for the Kampé de Fériet function" Inter. Conf. Ceramics, Intern. J. Modern Physics: Conf. Series, vol. 22, pp. 713-719, 2013, DOI: http://doi.org/10.1142/S2010194513010908.

[14] P. Agarwal, M. Chand, “Graphical analysis of Kampé De Fériet's series with implementation of MATLAB," Intern. J. Comp. Applic. (0975-8887), vol. 59, no. 4, Dec. 2012.
[15] Anon, "Incomplete Gamma function," Wikipedia, the free encyclopedia, Dec.

2015 , http://en.wikipedia.org/wiki/Incomplete_gamma_function.

[16]H. Bateman, Higher Transcendental Functions, vols. I, II, and III, (California Institute of Technology Bateman Manuscript Project, Dir. Arthur Erdélyi et al.) New York: McGraw-Hill, 1953-1955.

[17]I. Progri, Indoor Geolocation Systems-Theory and Applications. Vol. I, $1^{\text {st }}$ ed., Worcester, MA: Giftet Inc., $\sim 800$ pp., 2019 (not yet available in print).

[18]H.M. Srivastava, P.W. Karlsson, Multiple Gaussian Hypergeometric Series. Horwood, Chichester, 1985.

[19]H.M. Srivastava, "Hypergeometric functions of three variables," Ganita vol. 15, pp. 97-108, 1964.

[20]H.M. Srivastava, H.L., Monacha, A Treatise on Generating Functions. Horwood, Chichester, 1984.

[21]H.M. Srivastava, "Some integrals representing triple hypergeometric series," Rend. Circ. Mat. Palermo, vol. 16, no. 1, pp. 99-115, 1967.

[22]C. Wei, X. Wang, Y. Li, "Certain transformations for multiple hypergeometric functions," Adv. in Differ. Equ., 13 pg., 2013, 2013-360, DOI: http://doi.org/10.1186/16871847-2013-360.

[23] S. Gaboury, R. Tremblay, "Remark on certain transformations for multiple hypergeometric functions. Adv. Diff. Equat., 12 pg., 2014, http://doi.org/10.1186/1687-1847-2014-126.

[24]I.S. Gradshteyn, I.M. Ryzhik, (A. Jeffrey, D. Zwillinger, editors) Table of Integrals, Series, and Products, 7th ed., Burlington, MA: Academic Press, 1171 pp., 2007.

[25]G.B. Arfken, H.J., Weber, Mathematical Methods for Physicists, San Diego, CA: Academic Press, 1995.

[26]Anon, "Gamma function," Wikipedia, the free encyclopedia, 2015, https://en.wikipedia.org/wiki/Gamma_function.

[27]Anon, "Hypergeometric function," Wikipedia, the free encyclopedia, Dec.

2015 , https://en.wikipedia.org/wiki/Hypergeometric_function.

[28]Anon, “L'Hôpital's rule," Wikipedia, the free encyclopedia, Feb. 2018, https://en.wikipedia.org/wiki/L\%27H\%C3\%B4pital\%27s rule.

[29]Anon, "Pochhammer symbol," Wikipedia, the free encyclopedia, Dec. 2015 , https://en.wikipedia.org/wiki/Pochhammer_symbol. 
[30] Anon, "MATLAB 2018b," The MathWorks, Inc., Natick, MA, Copyright (C) 1994-2018, The MathWorks, Inc., https://mathworks.com/products/matlab.html.

[31] Anon, “Generalized Hypergeometric function," Wikipedia, the free encyclopedia, June, 2017, https://en.wikipedia.org/wiki/Generalized_hypergeometri c function.

\section{Appendix A: Inefficient Implementation of $\mathcal{F}_{21}(x ; a, 0,0)$}

There is another inefficient algorithm for computing $\mathcal{F}_{21}(x ; a, 0,0)$. The main reason for showing this is to make sure that scholars understand the difference of both algorithms.

From Progri (2016, [4] (127)), we have $\psi(k+1)$

$$
\psi(k+1)=\frac{k}{k+1} F\left[\begin{array}{c}
k+1,1,1 ; 1 \\
k+2,2 ;
\end{array}\right]+\psi(1)
$$

Substituting (164) into (96) yields

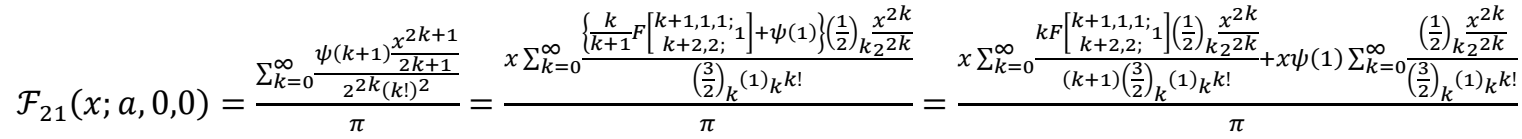

The second summation of (165) can be computed from It remains to compute the first summation as follows generalized hypergeometric function [31].

$$
\begin{aligned}
x \psi(1) & {\left[\sum_{k=0}^{\infty} \frac{\left(\frac{1}{2}\right)_{k} \frac{x^{2 k}}{2^{2 k}}}{\left(\frac{3}{2}\right)_{k}(1)_{k} k !} \equiv x_{1} F_{2}\left(\frac{1}{2} ; \frac{3}{2}, 1 ; x_{1}\right)\right] \quad(166) } \\
& \sum_{k=0}^{\infty} \frac{k F\left[\begin{array}{c}
k+1,1,1 ; 1 \\
k+2,2 ;
\end{array}\right]\left(\frac{1}{2}\right)_{k} \frac{x^{2 k}}{2^{2 k}}}{(k+1)\left(\frac{3}{2}\right)_{k}(1)_{k} k !}=\sum_{k=0}^{\infty} \frac{k \sum_{m=0}^{\infty} \frac{(k+1)_{m}(1)_{m}(1)_{m} m}{(k+2)_{m}(2)_{m} m !}\left(\frac{1}{2}\right)_{k} \frac{x^{2 k}}{2^{2 k}}}{(k+1)\left(\frac{3}{2}\right)_{k}(1)_{k} k !}=\sum_{k=1}^{\infty} \frac{k \sum_{m=0}^{\infty} \frac{(k+1)_{m}(1)_{m}(1)_{m} 1^{m}}{(k+2)_{m}(2)_{m}}\left(\frac{1}{m !}\right)_{k} \frac{x^{2 k}}{(k+1)\left(\frac{3}{2}\right)_{k}(1)_{k} k !}}{(k !}
\end{aligned}
$$

Which is identical to

$$
\sum_{k=0}^{\infty} \frac{k F\left[\begin{array}{c}
k+1,1,1 ; \\
k+2,2 ;
\end{array}\right]\left(\frac{1}{2}\right)_{k} \frac{x^{2 k}}{2^{2 k}}}{(k+1)\left(\frac{3}{2}\right)_{k}(1)_{k} k !}=\sum_{k=0}^{\infty} \frac{(k+1) \sum_{m=0}^{\infty} \frac{(k+2)_{m}(1)_{m}(1)_{m}{ }^{m}}{(k+3)_{m}(2)_{m} m !}\left(\frac{1}{2}\right)_{k+1} \frac{x^{2(k+1)}}{(k+2)\left(\frac{3}{2}\right)_{k+1}(1)_{k+1}(k+1) !}}{(k+1)}=\frac{x^{2}}{2^{2}} \frac{1}{6} \sum_{k=0}^{\infty} \sum_{m=0}^{\infty} \frac{(2)_{k+m}\left(\frac{3}{2}\right)_{k}(1)_{m}(1)_{m}}{(3)_{k+m}\left(\frac{5}{2}\right)_{k}(2)_{k}(2)_{m}} \frac{\left(\frac{x^{2}}{2^{2}}\right)^{k}}{k !} \frac{1^{m}}{m !}
$$

Finally, Dr. Progri has successfully derived for the first time Kampé de Fériet function [11]-[13] as follows that this summation which can be written with the help of the

$$
\sum_{k=0}^{\infty} \frac{k F\left[\begin{array}{c}
k+1,1,1 ; 1 \\
k+2,2 ; 1
\end{array}\right]\left(\frac{1}{2}\right)_{k} \frac{x^{2 k}}{2^{2 k}}}{(k+1)\left(\frac{3}{2}\right)_{k}(1)_{k} k !}=\frac{x^{2}}{2^{2}} \frac{1}{6} \sum_{k=0}^{\infty} \sum_{m=0}^{\infty} \frac{(2)_{k+m}\left(\frac{3}{2}\right)_{k}(1)_{m}(1)_{m}}{(3)_{k+m}\left(\frac{5}{2}\right)_{k}(2)_{k}(2)_{m}} \frac{\left(\frac{x^{2}}{2^{2}}\right)^{k}}{k !} \frac{1^{m}}{m !}=\frac{x^{2}}{2^{2}} \frac{1}{6} F_{1: 2 ; 1}^{1: 1 ; 2}\left[\begin{array}{l}
2: \frac{3}{2} ; 1,1 ; \\
3: \frac{5}{2}, 2 ; 2 ;
\end{array}\right]
$$

Substituting (166) and (169) into (165)

$$
\mathcal{F}_{21}(x ; a, 0,0)=\frac{\sum_{k=0}^{\infty} \frac{\psi(k+1) \frac{x^{2 k+1}}{2 k+1}}{2^{2 k}(k !)^{2}}}{\pi}=\frac{\frac{x^{3} 1}{2^{3} 3} F_{1: 2 ; 1}^{1: 1 ; 2}\left[\begin{array}{l}
2: \frac{3}{2} ; 1,1 ; \\
3: \frac{5}{2}, 2 ; 2 ;
\end{array} x_{1}, 1\right]+x \psi(1)_{1} F_{2}\left(\frac{1}{2} ; \frac{3}{2}, 1 ; x_{1}\right)}{\pi}
$$

Equation (170) is just as accurate as (96) but is computationally much more expensive; hence, it is not recommended in implementation.

\section{Appendix B: Alternative Implementation of $\mathcal{F}_{\text {GBessel2 }}(\boldsymbol{x} ; \boldsymbol{a}, 0, \boldsymbol{p})$}

I initially developed the alternative implementation of $\mathcal{F}_{\mathrm{GBessel} 2}(x ; a, 0, p)$ then later on I discovered the recursive algorithm which is discussed in Sect. 3.2. It is also important to note that both the recursive approach and the direct computation algorithm, discussed here, produce similar performance in terms of accuracy and computational load. The only drawback to the direct approach is that it is more complex than the recursive algorithm.

When $d=0$ and $p$ a positive, $f_{\mathrm{GBessel} 2}(x ; a, 0, p)$ is given by (34).

Following from Progri 2016 [1], (50)-(53), substituting (31) 
into (121) of Progri 2016 [1]

$$
\mathcal{F}_{\mathrm{GBessel} 2}(x ; a, 0, p)=\frac{\int_{0}^{x} t^{p}\left[\frac{1}{2} \sum_{k=0}^{p-1(-1)^{k}(p-k-1) !}\left(\frac{t}{2}\right)^{2 k-p}+(-1)^{p} \sum_{k=0}^{\infty} \frac{\frac{\psi(k+1)+\psi(p+k+1)}{2}-\ln \frac{t}{2}}{k !(p+k) !}\left(\frac{t}{2}\right)^{p+2 k}\right] d t}{C_{2}(p, 0)}
$$

Changing the order of summation and integration we obtain

$$
\mathcal{F}_{\mathrm{GBesse} 2}(x ; a, d, p)=\frac{2^{p-1} \sum_{k=0}^{p-1} \frac{(-1)^{k}(p-k-1) ! \int_{0}^{x} t^{2 k} d t}{k ! 2^{2 k}}+\frac{(-1) p}{2^{p}} \sum_{k=0}^{\infty} \frac{[\psi(k+1)+\psi(p+k+1)] \int_{0}^{x} t^{2 p+2 k} d t}{k !(p+k) ! 2^{2 k}}+\frac{(-1) p_{2}}{2^{p}} \sum_{k=0}^{\infty} \frac{\int_{0}^{x} \ln \frac{t}{2} t^{2 p+2 k} d t}{k !(p+k) ! 2^{2 k}}}{C_{2}(p, d)}
$$

The first integral is pretty straightforward to compute (see 90).

We start with the first summation. Substituting, (90) into the

$$
\mathcal{F}_{21}(x ; a, 0, p)=\frac{\sum_{k=0}^{p-1} \frac{(p-k-1) ! \frac{x^{2 k+1}}{2 k+1}}{(-1)^{-k_{2}{ }^{2 k} k !}}}{2^{1-p_{C_{2}}(p, 0)}}=\mid \begin{array}{cc}
\frac{x 2^{p-1} \sum_{k=0}^{p-1} \frac{(p-k-1) !\left(\frac{1}{2}\right)_{k}\left(-x_{1}\right)^{k}}{\left(\frac{3}{2}\right)_{k} k !}}{C_{2}(p, 0)} & p \neq 0 \\
0 & p=0
\end{array}
$$

Equation (173) can be written with the help of the modified

generalized hypergeometric function as follows

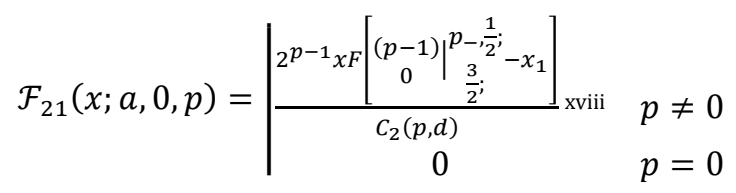

The second integral is also pretty straightforward to compute

Substituting (175) into the second summation of (172) yields

$$
\begin{aligned}
& \int_{0}^{x} t^{2 p+2 k} d t=\frac{x^{2 p+2 k+1}}{2 p+2 k+1} \\
& \sum_{k=0}^{\infty} \frac{[\psi(k+1)+\psi(p+k+1)] \int_{0}^{x} t^{2 p+2 k} d t}{k !(p+k) ! 2^{2 k}}=\sum_{k=0}^{\infty} \frac{[\psi(k+1)+\psi(p+k+1)] \frac{x^{2 p+2 k+1}}{2 p+2 k+1}}{2^{2 k} k !(p+k) !}
\end{aligned}
$$

Substituting (176) into the second summation of (172) yields,

$$
\mathcal{F}_{22}(x ; a, d, p)=\frac{(-1)^{p}}{2^{p_{2}}} \frac{\sum_{k=0}^{\infty} \frac{[\psi(k+1)+\psi(p+k+1)] \frac{x^{2 p+2 k+1}}{2 p+2 k+1}}{2^{2 k}(k !)^{2}}}{C_{2}(p, d)}=\frac{(-1)^{p}}{2^{p_{2}}} \frac{[\psi(1)+\psi(p+1)] \frac{x^{2 p+1}}{2 p+1}+x^{2 p+1} \sum_{k=1}^{\infty} \frac{[\psi(k+1)+\psi(p+k+1)] x_{1}^{k}}{(2 p+2 k+1) k !(p+k) !}}{C_{2}(p, d)}
$$

Where, here we use the continued fraction expansion of the rational function; hence, we have

$$
\mathcal{F}_{22}(x ; a, d, p)=\frac{(-1)^{p} x^{2 p_{1}}}{2^{p_{4}} p_{1} \Gamma\left(p_{2}\right)} \frac{\psi(1)+\psi(p+1)+\sum_{k=1}^{\infty} \frac{[\psi(k+1)+\psi(p+k+1)]\left(p_{1}\right)_{k} x_{1}^{k}}{\left(p_{3}\right)_{k}\left(p_{2}\right)_{k}} k !}{C_{2}(p, d)}
$$

Equation (178) can be computed recursively.

The second integral is more difficult to compute directly, so

The third summation function of (172) can be computed we integrate by parts from the third integral.

$$
\int_{0}^{x} \ln \frac{t}{2} t^{2 p+2 k} d t=\left[\left.\ln \left(\frac{t}{2}\right) \frac{t^{2 p+2 k+1}}{2 p+2 k+1}\right|_{0} ^{x}=\ln \left(\frac{x}{2}\right) \frac{x^{2 p+2 k+1}}{2 p+2 k+1}-\lim _{x \rightarrow 0} \ln \left(\frac{x}{2}\right) \frac{x^{2 p+2 k+1}}{2 p+2 k+1}\right]-\frac{1}{2 p+2 k+1} \int_{0}^{x} t^{2 p+2 k} d t
$$

The lower limit of $\ln \left(\frac{x}{2}\right) \frac{x^{2 p+2 k+1}}{2 p+2 k+1} \quad k=0,1, \cdots$ can be

$$
\lim _{x \rightarrow 0} \ln \left(\frac{x}{2}\right) \frac{x^{2 p+2 k+1}}{2 p+2 k+1}=-\frac{2 p+2 k+2}{2 p+2 k+1} \lim _{x \rightarrow 0} \frac{x^{-1}}{x^{-2 p-2 k-2}}=-\frac{2 p+2 k+2}{2 p+2 k+1} \lim _{x \rightarrow 0} x^{2 p+2 k+2}=0
$$


Substituting (179), (180) into the second summation of (172) yields

$$
\mathcal{F}_{23}(x ; a, d, p)+\mathcal{F}_{24}(x ; a, d, p)=\frac{\sum_{k=0}^{\infty} \frac{\int_{0}^{x} \ln \frac{t}{2} t^{2 p+2 k} d t}{2^{2 k} k !(p+k) !}}{C_{2}(p, d)}=\frac{\ln \left(\frac{x}{2}\right) \sum_{k=0}^{\infty} \frac{\frac{x^{2 p+2 k+1}}{2 p+2 k+1}}{2^{2 k} k !(p+k) !}-\sum_{k=0}^{\infty} \frac{\frac{1}{2 p+2 k+1} \int_{0}^{x} t^{2 p+2 k} d t}{2^{2 k} k !(p+k) !}}{C_{2}(p, d)}
$$

Next, we work on the first summation of (181). Substituting

(175) into the second summation yields

$$
\sum_{k=0}^{\infty} \frac{\frac{x^{2 p+2 k+1}}{2 p+2 k+1}}{2^{2 k} k !(p+k) !}=\frac{x^{2 p_{1}}}{2 \Gamma\left(p_{2}\right)} \frac{1}{p_{1}} \sum_{k=0}^{\infty} \frac{\left(p_{1}\right)_{k} \frac{x_{1}^{k}}{\left(p_{3}\right)_{k}\left(p_{2}\right)_{k}}}{\left(p_{1}\right.}
$$

Equation (182) can be written with the help of the generalized hypergeometric function [31] as follows

$$
\sum_{k=0}^{\infty} \frac{\frac{x^{2 p+2 k+1}}{2 p+2 k+1}}{2^{2 k} k !(p+k) !}=\frac{x^{2 p_{1}}}{2 p_{1} \Gamma\left(p_{2}\right)} F\left[p_{1} ; p_{3}, p_{2} ; x_{1}\right]
$$

Substituting (183) into the first summation of (181) yields,

$$
\mathcal{F}_{23}(x ; a, d, p)=\frac{\frac{(-1) p_{2}}{2^{p}} \ln \left(\frac{x}{2}\right) \sum_{k=0}^{\infty} \frac{\frac{x^{2 p+2 k+1}}{2 p+2 k+1}}{2^{2 k} k !(p+k) !}}{C_{2}(p, d)}=\frac{\ln \left(\frac{x}{2}\right) \frac{(-1) p_{2} x^{2} p_{1}}{2^{2} p_{1} \Gamma\left(p_{2}\right)} F\left[p_{1} ; p_{3}, p_{2} ; x_{1}\right]}{C_{2}(p, d)}
$$

For the computation of the second summation of (181) we and after performing the integration we obtain employ the series expansion of the incomplete gamma function

$$
\mathcal{F}_{24}(x ; a, d, p)=\frac{\frac{(-1) p}{2^{p}} \sum_{k=0}^{\infty} \frac{\frac{x^{2 k+2 p_{1}}}{\left(2 k+2 p_{1}\right)^{2}}}{2^{2 k} k !(p+k) !}}{C_{2}(p, d)}=\frac{\frac{(-1)_{2}}{2^{p_{4} p_{1} p_{1}}} x^{2 p_{1}} \sum_{k=0}^{\infty} \frac{\left(p_{1}\right)_{k}\left(p_{1}\right)_{k} \frac{x_{1}^{k}}{k !}}{\left.c_{2}\right)_{k}\left(p_{3}\right)_{k}\left(p_{2}\right)_{k}}}{C_{2}(p, d)}
$$

Equation (185) can be written with the help of the generalized hypergeometric function, ${ }_{2} F_{3},[31]$ as follows

$$
\mathcal{F}_{24}(x ; a, d, p)=\frac{(-1)^{p}}{{ }^{p_{4}} p_{1}^{2}} \frac{x^{2 p_{1}}}{\Gamma\left(p_{2}\right) C_{2}(p, d)}{ }_{2} F_{3}\left[p_{1}, p_{1} ; p_{3}, p_{3}: p_{2} ; x_{1}\right]
$$

Finally, substituting (174), (178), (184) and (186) into (172) $\quad \mathcal{F}_{\mathrm{GBessel} 2}(x ; a, 0, p)$ produces the desired closed form expression of

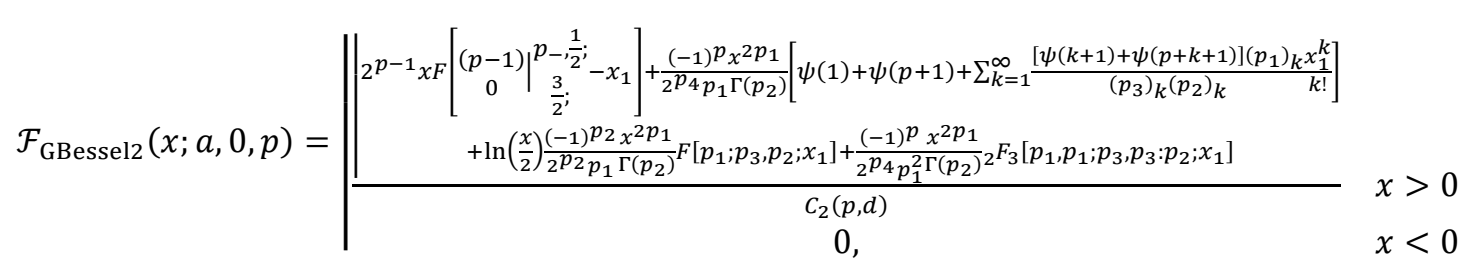

Equation (187) is an original expression not published anywhere else; however, care must be shown in the computation of generalized hypergeometric functions [31].

The obvious question is as follows. Is (187) the right equation? While numerically we will test this at the numerical, theoretical results section, we can still test analytically for at least one data point: (1) $d=0, p=0$.

Hence, for $d=0, p=0$ from (187), we obtain.

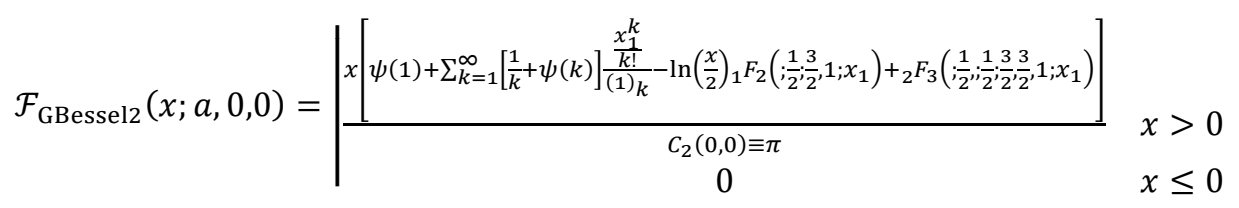

Equation (188) is identical to (98); thus, completing the proof for $d=0, p=0$.

This concludes the derivations of the special case for $d=0$ and $p \neq 0, p$ a positive integer.

\section{Appendix C: Inefficient Implementation of $\mathcal{F}_{23}(x ; a, d, 0)$}

If we were to employ the continued fraction expansion of the 
incomplete gamma function in $\mathcal{F}_{23}(x ; a, d, 0)$ we have

The second integral is equal to

$$
\int_{0}^{x} \frac{t^{-1} \gamma(2 k+1, t d)}{d^{2 k+1}} d t=\int_{0}^{x} t^{-1} \frac{t^{2 k+1} e^{-d t}}{(2 k+1)}\left[\sum_{m=0}^{\infty} \frac{\left(\frac{d^{2} t^{2}}{2^{2}}\right)^{m}}{\left(k+\frac{3}{2}\right)_{m}(k+1)_{m}}+\frac{d t}{2(k+1)} \sum_{m=0}^{\infty} \frac{\left(\frac{d^{2} t^{2}}{2^{2}}\right)^{m}}{(k+2)_{m}\left(k+\frac{3}{2}\right)_{m}}\right] d t
$$

Which is equivalent with

$$
\int_{0}^{x} \frac{t^{-1} \gamma(2 k+1, t d)}{d^{2 k+1}} d t=\frac{\left[\sum_{m=0}^{\infty} \frac{\int_{0}^{x} t^{2 k+2 m} e^{-d t} d t\left(\frac{d^{2}}{2^{2}}\right)^{m}}{\left(k+\frac{3}{2}\right)_{m}(k+1) m}+\frac{d}{2(k+1)} \sum_{m=0}^{\infty} \frac{\int_{0}^{x} t^{2 k+2 m+1} e^{-d t} d t\left(\frac{d^{2}}{2^{2}}\right)^{m}}{(k+2)_{m}\left(k+\frac{3}{2}\right)_{m}}\right]}{(2 k+1)}
$$

Utilizing the result from Progri 2016 [1], (81) we obtain

$$
\int_{0}^{x} \frac{t^{-1} \gamma(2 k+1, t d)}{d^{2 k+1}} d t=\frac{\left[\sum_{m=0}^{\infty} \frac{\frac{\gamma(2 k+2 m+1, d x)}{d^{2 k+2 m+1}}\left(\frac{d^{2}}{2^{2}}\right)^{m}}{\left(k+\frac{3}{2}\right)_{m}{ }^{(k+1) m}}+\frac{d}{2(k+1)} \sum_{m=0}^{\infty} \frac{\frac{\gamma(2 k+2 m+2, d x)}{d^{2 k+2 m+2}}\left(\frac{d^{2}}{2^{2}}\right)^{m}}{(k+2)_{m}\left(k+\frac{3}{2}\right)_{m}}\right]}{(2 k+1)}
$$

Finally, substituting (191) into (117) yields,

$$
\mathcal{F}_{23}(x ; a, d, 0)=\frac{\sum_{k=0}^{\infty} \frac{\left[\sum_{m=0}^{\infty} \frac{\gamma(2 k+2 m+1, d x)\left(\frac{1}{2^{2}}\right)^{m}}{\left.\left(k+\frac{3}{2}\right)_{m}{ }^{(k+1) m}+\frac{1}{2(k+1)} \sum_{m=0}^{\infty} \frac{\gamma(2 k+2 m+2, d x)\left(\frac{1}{2^{2}}\right)^{m}}{(k+2) m\left(k+\frac{3}{2}\right)_{m}}\right]}\right.}{d^{2 k+1}(2 k+1) 2^{2 k}(k !)^{2}}}{C_{2}(0, d)}
$$

Which is identical to

$$
\mathcal{F}_{23}(x ; a, d, 0)=\frac{\sum_{k, m=0}^{\infty} \frac{\frac{\gamma(2 k+2 m+1, d x)}{\left.d^{2 k+1} 2^{2 k+2 m}(k !)\right)^{2}}}{\left(k+\frac{3}{2}\right)_{m}(k+1)_{m}(2 k+1)}+\frac{1}{2} \sum_{k, m=0}^{\infty} \frac{\frac{\gamma(2 k+2 m+2, d x)}{d^{2 k+1} 2^{2 k+2 m}(k !)^{2}}}{(k+2) m\left(k+\frac{3}{2}\right)_{m}{ }^{(k+1)(2 k+1)}}}{C_{2}(0, d)}
$$

Next, we work on the first summation of (193). Substituting

(19) into the third summation yields, an expansion of $\gamma(2 k+2 m+1, d x)$ from Progri 2018, [2]

$$
\sum_{k, m=0}^{\infty} \frac{\frac{\gamma(2 k+2 m+1, d x)}{d^{2 k+1} 2^{2 k+2 m}(k !)^{2}}}{\left(k+\frac{3}{2}\right)_{m}{ }^{(k+1)_{m}(2 k+1)}}=\sum_{k, m=0}^{\infty} \frac{\frac{(d x)^{2 k+2 m+1} e^{-d x}}{(2 k+2 m+1)}\left[\sum_{n=0}^{\infty} \frac{x_{2}^{n}}{\left(k+m+\frac{3}{2}\right)_{n}{ }^{(k+m+1)} n}+\frac{d x}{2(k+m+1)} \sum_{n=0}^{\infty} \frac{x_{2}^{n}}{(k+m+2)_{n}\left(k+m+\frac{3}{2}\right)_{n}}\right]}{d^{2 k+1} 2^{2 k+2 m}(k !)^{2}}
$$

Which is equivalent with

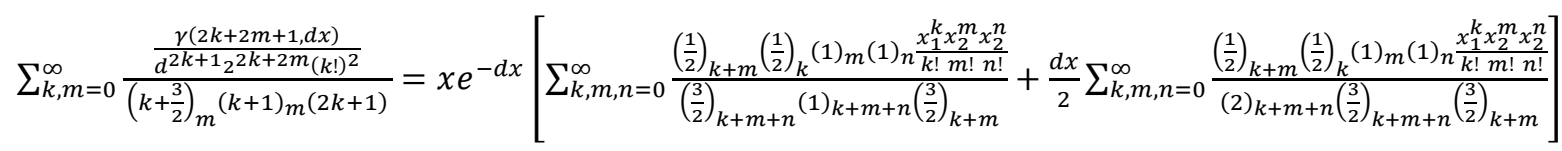

Equation (195) can be written efficiently by the help of the Appendix E) as follows Srivastava's Triple Hypergeometric Series $F^{(3)}[x, y, z]$ (see

$$
\mathcal{F}_{231}(x ; a, d, 0)=\frac{\sum_{k, m=0}^{\infty} \frac{\frac{\gamma(2 k+2 m+1, d x)}{d^{2 k+1} 2^{2 k+2 m}(k !)^{2}}}{\left(k+\frac{3}{2}\right)_{m}{ }^{(k+1) m}(2 k+1)}}{C_{2}(0, d)}=\frac{x e^{-d x}\left[F^{(3)}\left[\begin{array}{c}
-:: \frac{1}{2} ;-;-: \frac{1}{2} ; 1 ; 1 ; \\
\frac{3}{2}, 1: \frac{3}{2} ;-;-:-;-;-;
\end{array} x_{1}, x_{2}, x_{2}\right]+\frac{d x}{2} F^{(3)}\left[\begin{array}{c}
-:: \frac{1}{2} ;-;-: \frac{1}{2} ; 1 ; 1 ; \\
2, \frac{3}{2}: \frac{3}{2} ;-;-;-;-;-;
\end{array} x_{1}, x_{2}, x_{2}\right]\right]}{C_{2}(0, d)}
$$

The last summation can also be written by means of the of the Srivastava's Triple Hypergeometric Series $F^{(3)}[x, y, z](\operatorname{see}$ Appendix E).

$$
\frac{1}{2} \sum_{k, m=0}^{\infty} \frac{\frac{\gamma(2 k+2 m+2, d x)}{d^{2 k+1} 2^{2 k+2 m}(k !)^{2}}}{(k+2)_{m}\left(k+\frac{3}{2}\right)_{m}{ }^{(k+1)(2 k+1)}}=\frac{1}{2} \sum_{k, m=0}^{\infty} \frac{\frac{\frac{(d x)^{2 k+2 m+2} e^{-d x}}{(2 k+2 m+2)}\left[\sum_{n=0}^{\infty} \frac{x_{2}^{n}}{(k+m+2)_{n}\left(k+m+\frac{3}{2}\right)_{n}}+\frac{d x}{2\left(k+m+\frac{3}{2}\right)} \sum_{n=0}^{\infty} \frac{x_{2}^{n}}{\left(k+m+\frac{5}{2}\right)_{n}(k+m+2)_{n}}\right]}{d^{2 k+1} 2^{2 k+2 m}(k !)^{2}}}{(k+2)_{m}\left(k+\frac{3}{2}\right)_{m}{ }^{(k+1)(2 k+1)}}
$$


Which is equivalent with

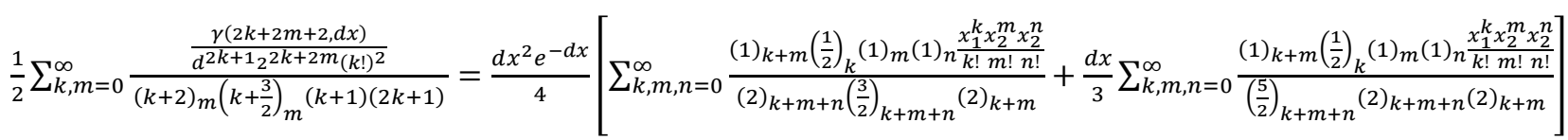

Equation (198) can be written efficiently by the help of the Appendix E) as follows Srivastava's Triple Hypergeometric Series $F^{(3)}[x, y, z]$ (see

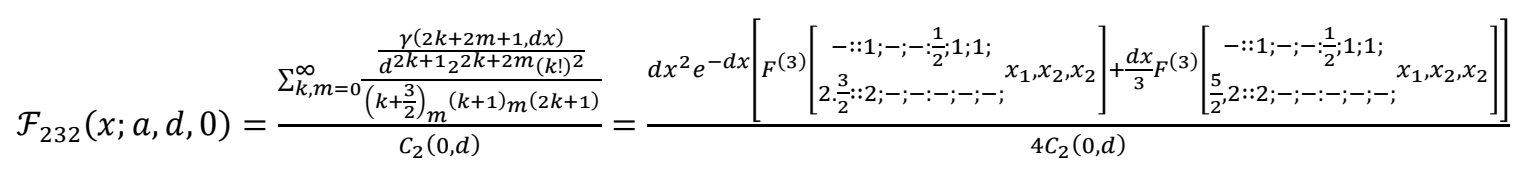

Finally, substituting (196) and (199) into (193) produces a computationally inefficient implementation of $\mathcal{F}_{23}(x ; a, d, 0)$

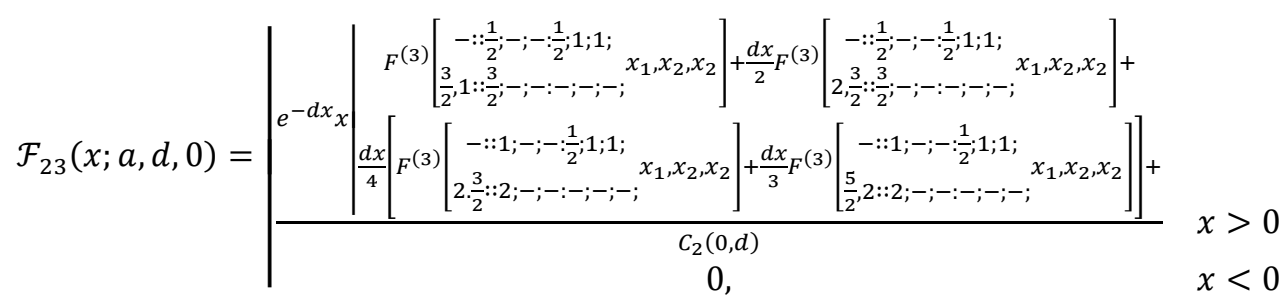

Equation (200) is an original expression not published anywhere else; however, care must be shown in the computation of the Srivastava's Triple Hypergeometric Series $F^{(3)}[x, y, z]$ (see Appendix F).

$$
\mathcal{F}_{23}(x ; a, d=0,0)=x \frac{F^{(3)}\left[\begin{array}{c}
-:: \frac{1}{2} ;-;-; \frac{1}{2} ; 1 ; 1 ; \\
\frac{3}{2}, 1: \frac{3}{2} ;-;-:-;-;-;
\end{array} x_{1} 0,0\right]}{C_{2}(0,0)}
$$

Evaluating the Srivastava's Triple Hypergeometric Series $\quad F^{(3)}[x, y, z][18]-[22]$ (see Appendix E) at $d=0$ yields

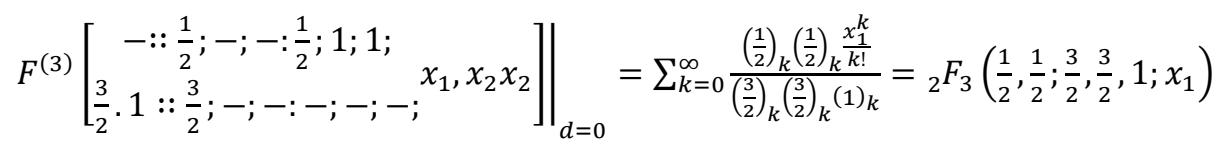

Substituting (202) into (200) yields

$$
\mathcal{F}_{\mathrm{GBessel} 2}(x ; a, 0,0)=x \frac{{ }_{2} F_{3}\left(\frac{1}{2}, \frac{1}{2} ; \frac{3}{2}, \frac{3}{2}, 1 ; x_{1}\right)}{C_{2}(0,0) \equiv \pi}
$$

Equation (203) is identical to (95); thus, completing the proof for $d=0$.

This concludes the derivations of the special case for $d \neq 0$ and $p=0$ via the inefficient implementation of $\mathcal{F}_{23}(x ; a, d, 0)$. recursively based on the recursive expansions of the incomplete gamma function; however, this approach is not as accurate as the expansion via the modified generalized the Kampé de Fériet double hypergeometric series developed by Progri 2019.

From (138) we have,

\section{Appendix D: Recursive Implementation of $\mathcal{F}_{22}(\boldsymbol{x} ; \boldsymbol{a}, \boldsymbol{d}, \boldsymbol{p})$}

The function $\mathcal{F}_{22}(x ; a, d, p)$ can also be implemented

$$
\mathcal{F}_{22}(x ; a, d, p)=\frac{(-1)^{p}}{2^{p}} \frac{\sum_{k=0}^{\infty} \frac{[\psi(k+1)+\psi(p+k+1)] \frac{\gamma(2 p+2 k+1, d x)}{d^{2} p+2 k+1}}{2^{2 k}(k !)^{2}}}{C_{2}(p, d)}=\frac{(-1)^{p}}{2^{p_{2}}} \frac{[\psi(1)+\psi(p+1)] \frac{\gamma(2 p+1, d x)}{d^{2} p+1}+\sum_{k=1}^{\infty} \frac{[\psi(k+1)+\psi(p+k+1)] \frac{\gamma(2 p+2 k+1, d x)}{d^{2} p+2 k+1}}{2^{2 k} k !(p+k) !}}{C_{2}(p, d)}
$$


Where, here we use the continued fraction expansion of the incomplete gamma function (see Progri 2018 [2], (1))

$$
\gamma\left(2 p_{1}, d x\right)=\frac{x^{2 p_{1} d^{2} p_{1} e^{-d x}}}{2 p_{1}} \Phi\left(1,2 p_{2} ; d x\right)
$$

$$
\gamma(\alpha, d x)=\frac{x^{\alpha} d^{\alpha} e^{-d x}}{\alpha \equiv 2 p+2 k+1} \Phi(1, \alpha+1 ; d x)
$$

Employing the continued fraction expansion for $\gamma\left(2 p_{1}, d x\right)$ (205), and $\gamma(\alpha, d x)$ (206) we have,

$$
\mathcal{F}_{22}(x ; a, d, p)=\frac{(-1)^{p} e^{-d x}}{2^{p_{2}}} \frac{[\psi(1)+\psi(p+1)] \frac{x^{2 p+1}}{2 p+1} \Phi(1,2+2 p ; d x)+x^{2 p} \sum_{k=1}^{\infty} \frac{[\psi(k+1)+\psi(p+k+1)] \frac{x_{1}^{k}}{\alpha \equiv 2 p+2 k+1} \Phi(1, \alpha+1 ; d x)}{k !(p+k) !}}{C_{2}(p, d)}
$$

Equation (206) can be computed recursively, $\forall k=1,2, \cdots$

$$
\gamma(\alpha, d x)=2 p_{2 k}{ }^{\mathrm{xix}} \gamma\left[2 p_{2 k}, d x\right]-(d x)^{2 p_{2 k}} e^{-d x}
$$

$$
x \Phi(1, \alpha+1 ; d x)=\mid \begin{array}{cc}
x & d=0 \\
\frac{\alpha[\Phi(1, \alpha ; d x)-1]}{d} & d \neq 0
\end{array}
$$

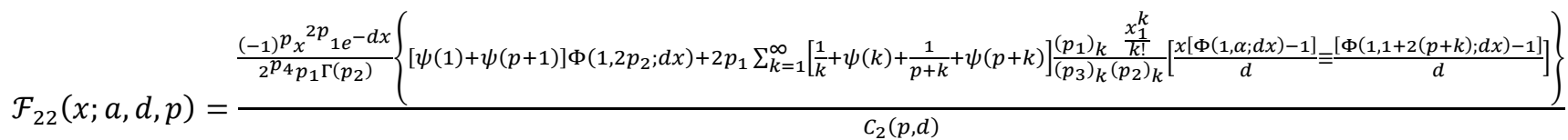

Equation (210) is an original algorithm for computation of $\mathcal{F}_{22}(x ; a, d, p)$ but it is several orders of magnitude less accurate than the direct computation via (142). However, in computational performance (210) and (142) are about the same.

\section{Appendix E: The Srivastava's Triple Hypergeometric Series $F^{(3)}[x, y, z]$}

It appears that the cdf of the Bessel function distribution of the second kind involves the use of Srivastava's triple

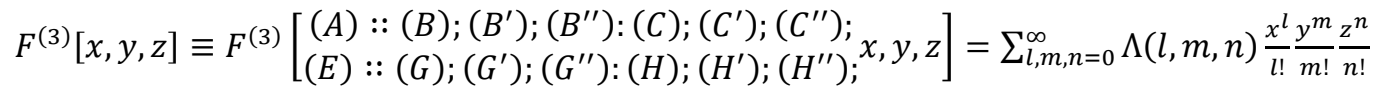

where $\Lambda(l, m, n)$ is given by

$$
\Lambda(l, m, n) \equiv \frac{\left(\left(A_{a}\right)\right)_{l+m+n}}{\left(\left(E_{e}\right)\right)_{l+m+n}} \cdot \frac{\left(\left(B_{b} ; B_{b \prime}^{\prime} ; B_{b \prime \prime}^{\prime \prime}\right)\right)_{l+m ; m+n ; l+n}}{\left(\left(G_{g} ; G_{g}^{\prime} ; G_{g^{\prime \prime}}^{\prime \prime}\right)\right)_{l+m ; m+n ; l+n}} \cdot \frac{\left(\left(C_{c} ; C_{c^{\prime}}^{\prime} ; C_{c^{\prime \prime}}^{\prime \prime}\right)\right)_{l ; m ; n}}{\left(\left(H_{h} ; H_{h \prime}^{\prime} ; H_{h^{\prime \prime}}^{\prime \prime}\right)\right)_{l ; m ; n}}
$$

where $\left(\left(B_{b} ; B_{b \prime}^{\prime} ; B_{b \prime \prime}^{\prime \prime}\right)\right)_{l ; m ; n}$ denotes a triple generalized

Pochhammer symbol as defined by

$$
\left(\left(B_{b} ; B_{b^{\prime}}^{\prime} ; B_{b_{\prime \prime}^{\prime \prime}}^{\prime \prime}\right)\right)_{l ; m ; n}=\left(\left(B_{b}\right)\right)_{l}\left(\left(B_{b \prime}^{\prime}\right)\right)_{m}\left(\left(B_{b^{\prime \prime}}^{\prime \prime}\right)\right)_{n}^{\mathrm{xx}}(213)
$$

An efficient implementation of

$$
F^{(3)}[x, y, z]=\sum_{l=0}^{\infty} \frac{\left(\left(C_{c}\right)\right)_{l}}{\left(\left(H_{h}\right)\right)_{l}} \frac{x^{l}}{l !} \sum_{m=0}^{\infty} \frac{\left(\left(B_{b}\right)\right)_{l+m}}{\left(\left(G_{g}\right)\right)_{l+m}} \frac{\left(\left(C_{c^{\prime}}^{\prime}\right)\right)_{m}}{\left(\left(H_{h \prime}^{\prime}\right)\right)_{m}} \frac{y^{m}}{m !} \sum_{n=0}^{\infty} \frac{\left(\left(A_{a}\right)\right)_{l+m+n}}{\left(\left(E_{e}\right)\right)_{l+m+n}} \frac{\left(\left(B_{b^{\prime}}^{\prime}\right)\right)_{m+n}\left(\left(B_{b^{\prime \prime}}^{\prime \prime}\right)\right)_{l+n}}{\left(\left(G_{g^{\prime}}^{\prime}\right)\right)_{m+n}\left(\left(G_{g^{\prime \prime}}^{\prime \prime}\right)\right)_{l+n}} \frac{\left(\left(C_{c^{\prime \prime}}^{\prime \prime}\right)\right)_{n}}{\left(\left(H_{h^{\prime \prime}}^{\prime \prime}\right)\right)_{n}} \frac{z^{n}}{n !}
$$

If we set $x=0$ then the $F^{(3)}[x, y, z]$ is reduced to a Kampé de Fériet double hypergeometric series as follows

$$
F^{(3)}[0, y, z]=\sum_{m=0}^{\infty} \frac{\left(\left(B_{b}\right)\right)_{m}}{\left(\left(G_{g}\right)\right)_{m}} \frac{\left(\left(C_{c^{\prime}}^{\prime}\right)\right)_{m}}{\left(\left(H_{h^{\prime}}^{\prime}\right)\right)_{m}} \frac{y^{m}}{m !} \sum_{n=0}^{\infty} \frac{\left(\left(A_{a}\right)\right)_{m+n}}{\left(\left(E_{e}\right)\right)_{m+n}} \frac{\left(\left(B_{b^{\prime}}^{\prime}\right)\right)_{m+n}\left(\left(B_{b^{\prime \prime}}^{\prime \prime}\right)\right)_{n}}{\left(\left(G_{g^{\prime}}^{\prime}\right)\right)_{m+n}\left(\left(G_{g^{\prime \prime}}^{\prime \prime}\right)\right)_{n}} \frac{\left(\left(C_{c^{\prime \prime}}^{\prime \prime}\right)\right)_{n}}{\left(\left(H_{h^{\prime \prime}}^{\prime \prime}\right)\right)_{n}} \frac{z^{n}}{n !}
$$


Which can be written in compact form as

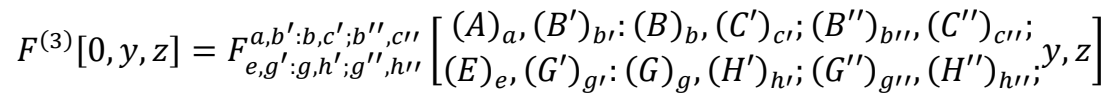

This concludes the discussion on the Srivastava's Triple Hypergeometric Series $F^{(3)}[x, y, z]$. Next we discuss a superefficient implementation of the Srivastava's triple hypergeometric series $F^{(3)}[x, y, z]$ based on the a supper efficient implementation algorithm of the Kampé de Fériet double hypergeometric series developed by Progri 2018 [2], Appendix A.

\section{Appendix F: A Super-Efficient Implementation of the Srivastava's Triple Hypergeometric Series $F^{(3)}[x, y, z]$}

The main purpose of this section is to develop a supper efficient implementation algorithm of the Srivastava's Triple Hypergeometric Series $F^{(3)}[x, y, z] \quad[18]-[23]$ that accomplishes the following three criteria: it is numerically very stable, computationally very efficient, and numerically very accurate; i.e., the Srivastava's triple hypergeometric series $F^{(3)}[x, y, z]$ [18]-[23] function numerical evaluation in MATLAB is no longer impacted by the numerical stability, efficiency, and accuracy. I hope that the Srivastava's triple hypergeometric series $F^{(3)}[x, y, z] \quad[18]-[23]$ function, presented in this appendix, can be implemented in MATLAB in like manner as the generalized hypergeometric series function hypergeom $(a, b, z)[30]$.

The Srivastava's triple hypergeometric series $F^{(3)}[x, y, z]$ [18]-[23] is defined as

$$
F^{(3)}[x, y, z]=\sum_{l=0}^{\infty} \frac{\left(\left(C_{c}\right)\right)_{l}}{\left(\left(H_{h}\right)\right)_{l}} \frac{x^{l}}{l !} \sum_{m=0}^{\infty} \frac{\left(\left(B_{b}\right)\right)_{l+m}}{\left(\left(G_{g}\right)\right)_{l+m}} \frac{\left(\left(C_{C^{\prime}}^{\prime}\right)\right)_{m}}{\left(\left(H_{h^{\prime}}^{\prime}\right)\right)_{m}} \frac{y^{m}}{m !} \sum_{n=0}^{\infty} \frac{\left(\left(A_{a}\right)\right)_{l+m+n}}{\left(\left(E_{e}\right)\right)_{l+m+n}} \cdot \frac{\left(\left(B_{b^{\prime}}^{\prime}\right)\right)_{m+n} \cdot\left(\left(B_{b^{\prime \prime}}^{\prime \prime}\right)\right)_{l+n}}{\left(\left(G_{g^{\prime}}^{\prime}\right)\right)_{m+n} \cdot\left(\left(G_{g^{\prime \prime}}^{\prime \prime}\right)\right)_{l+n}} \cdot \frac{\left(\left(C_{c^{\prime \prime}}^{\prime \prime}\right)\right)_{n}}{\left(\left(H_{h^{\prime \prime}}^{\prime \prime}\right)\right)_{n}} \frac{z^{n}}{n !}
$$

A super-efficient implementation of the Srivastava's triple hypergeometric series $F^{(3)}[x, y, z]$ [18]-[23] should be able to take advantage of the super-fast implementation of the generalized hypergeometric function [27], [31]; i.e., reduce the $F^{(3)}[x, y, z]$ [18]-[23] into the computation of a double summation.

For that we need to write the definition of the generalized hypergeometric series as follows [31] computation of the Srivastava's triple hypergeometric series

$$
{ }_{a} F_{c}\left[\begin{array}{l}
(A)_{a} \\
(C)_{c}
\end{array}\right]={ }_{a} F_{c}\left[\begin{array}{l}
A_{1}, \cdots, A_{a} \\
C_{1}, \cdots, C_{c}
\end{array}\right]={ }_{a} F_{c}\left[A_{1}, \cdots, A_{a} ; C_{1}, \cdots, C_{c} ; x\right]=\sum_{m \geq 0}^{\infty} \frac{\left(\left(A_{a}\right)\right)_{m}}{\left.\left(C_{c}\right)\right)_{m}} \frac{x^{m}}{m !}=\sum_{m \geq 0}^{\infty} \frac{\left(A_{1}\right)_{m} \cdots\left(A_{a}\right)_{m}}{\left(C_{1}\right)_{m} \cdots\left(C_{c}\right)_{m}} \frac{x^{m}}{m !}
$$

Employing a well-known identity of the Pochhammer symbol [29] into the formulation of the Srivastava's triple hypergeometric series $F^{(3)}[x, y, z][18]-[23]$ the latter one can be written as

$$
F^{(3)}[x, y, z]=\sum_{l, m, n \geq 0}^{\infty} \frac{\left(\left(C_{c}\right)\right)_{l}\left(\left(B_{b^{\prime \prime}}^{\prime \prime}\right)\right)_{l}}{\left(\left(H_{h}\right)\right)_{l}\left(\left(G_{g^{\prime \prime}}^{\prime \prime}\right)\right)_{l}} \frac{x^{l}}{l !} \frac{\left(\left(B_{b}\right)\right)_{l+m}\left(\left(A_{a}\right)\right)_{l+m}\left(\left(C_{c^{\prime}}^{\prime}\right)\right)_{m}\left(\left(B_{b^{\prime}}^{\prime}\right)\right)_{m}}{\left(\left(G_{g}\right)\right)_{l+m}\left(\left(E_{e}\right)\right)_{l+m}\left(\left(H_{h^{\prime}}^{\prime}\right)\right)_{m}\left(\left(G_{g^{\prime}}^{\prime}\right)\right)_{m}} \frac{y^{m}}{m !} \frac{\left(\left(A_{a}^{l m}\right)\right)_{n}\left(\left(B_{b^{\prime}}^{\prime m}\right)\right)_{n}\left(\left(B_{b^{\prime \prime}}^{\prime \prime l}\right)\right)_{n}\left(\left(C_{c^{\prime \prime}}^{\prime \prime}\right)\right)_{n}}{\left.\left(E_{e}^{l m}\right)\right)_{n}\left(\left(G_{g^{\prime}}^{\prime m}\right)\right)_{n}\left(\left(G_{g^{\prime \prime}}^{\prime \prime l}\right)\right)_{n}\left(\left(H_{h^{\prime \prime}}^{\prime \prime}\right)\right)_{n}} \frac{z^{n}}{n !}
$$

Where the following notation is used

$A_{a}^{l m}=A_{a}+l+m$

$$
B_{b^{\prime}}^{\prime m}=B_{b^{\prime}}^{\prime}+m
$$

Equation (219) can be split into the product of three summations

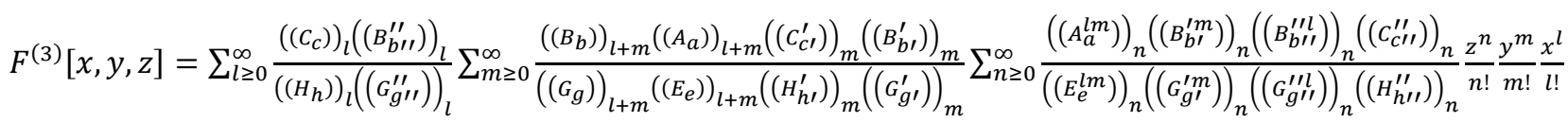

The inner hypergeometric series can be written as

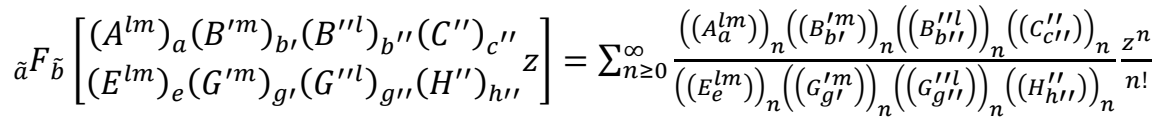


Where

$\tilde{a}=a, b^{\prime}, b^{\prime \prime}, c^{\prime \prime}$ and $\tilde{b}=e, g^{\prime}, g^{\prime \prime}, h^{\prime \prime}$
Substituting (225) into (224) yields a 2-dimensional supper fast and accurate summation of the Srivastava's triple hypergeometric series $F^{(3)}[x, y, z]$ [18]-[23] as follows

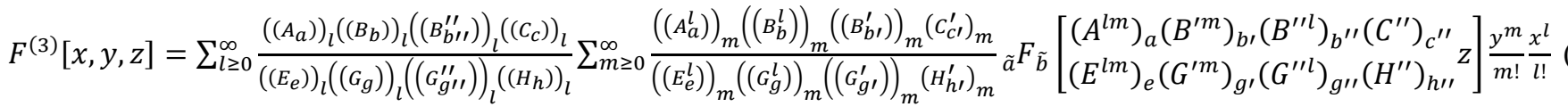

Next, we need a computationally efficient algorithm of

$\left(\left(A_{a}^{l}\right)\right)_{m},\left(\left(B_{b}^{l}\right)\right)_{m},\left(\left(B_{b \prime}^{\prime}\right)\right)_{m},\left(\left(C_{c \prime}^{\prime}\right)\right)_{m},\left(\left(E_{e}^{l}\right)\right)_{m},\left(\left(G_{g}^{l}\right)\right)_{m}$, $\left(\left(G_{g^{\prime}}^{\prime}\right)\right)_{m}$, and $\left(H_{h^{\prime}}^{\prime}\right)_{m}$

Let us consider for example the computation of $\left(\left(A_{a}\right)\right)_{m}$

$$
\left(\left(A_{a}^{l}\right)\right)_{m}=\prod_{i=1}^{a}\left(A_{i}^{l}\right)_{m}=\prod_{i=1}^{a} \frac{\Gamma\left(A_{i}^{l m}\right)}{\Gamma\left(A_{i}^{l}\right)}
$$

For large values of $m \gg 1$ and $A_{i}^{l} \gg 1, \Gamma\left(A_{i}^{l m}\right) \rightarrow \infty$; hence, an efficient computation of $\left(\left(A_{a}^{l}\right)\right)_{m} /\left(\left(E_{e}^{l}\right)\right)_{m}$ is required.

From the definition of the Pochhammer symbols $\left(A_{i}\right)_{l+m}$ [29] we have the following

$$
\left(A_{i}^{l}\right)_{m}=\prod_{k=0}^{m-1}\left(A_{i}^{l k}\right)
$$

Substituting (228) into (227) yields

$$
\left(\left(A_{a}^{l}\right)\right)_{m}=\prod_{i=1}^{a} \prod_{k=0}^{m-1}\left(A_{i}^{l k}\right)=\prod_{k=0}^{m-1} \prod_{i=1}^{a}\left(A_{i}^{l k}\right)
$$

Before we can perform the computation of (226) can we
Similarly we can write exactly the same formulas for $\left(\left(B_{b}^{l}\right)\right)_{m},\left(\left(B_{b^{\prime}}^{\prime}\right)\right)_{m},\left(\left(C_{c^{\prime}}^{\prime}\right)\right)_{m},\left(\left(E_{e}^{l}\right)\right)_{m},\left(\left(G_{g}^{l}\right)\right)_{m},\left(\left(G_{g^{\prime}}^{\prime}\right)\right)_{m}$, and $\left(H_{h^{\prime}}^{\prime}\right)_{m}$ as follows

$$
\begin{aligned}
& \left(\left(B_{b}^{l}\right)\right)_{m}=\prod_{i=1}^{b} \prod_{k=0}^{m-1}\left(B_{b}^{l k}\right)=\prod_{k=0}^{m-1} \prod_{i=1}^{b}\left(B_{b}^{l k}\right) \\
& \left(\left(B_{b^{\prime}}^{\prime}\right)\right)_{m}=\prod_{i=1}^{b \prime} \prod_{k=0}^{m-1}\left(B_{b^{\prime}}^{\prime k}\right)=\prod_{k=0}^{m-1} \prod_{i=1}^{b \prime}\left(B_{b^{\prime}}^{\prime k}\right) \\
& \left(\left(C_{c^{\prime}}^{\prime}\right)\right)_{m}=\prod_{i=1}^{c \prime} \prod_{k=0}^{m-1}\left(C_{i}^{\prime k}\right)=\prod_{k=0}^{m-1} \prod_{i=1}^{c^{\prime}}\left(C_{i}^{\prime k}\right) \\
& \left(\left(E_{e}^{l}\right)\right)_{m}=\prod_{i=1}^{e} \prod_{k=0}^{m-1}\left(E_{e}^{l k}\right)=\prod_{k=0}^{m-1} \prod_{i=1}^{e}\left(E_{e}^{l k}\right) \\
& \left(\left(G_{g}^{l}\right)\right)_{m}=\prod_{i=1}^{g} \prod_{k=0}^{m-1}\left(G_{g}^{l}\right)=\prod_{k=0}^{m-1} \prod_{i=1}^{g}\left(G_{g}^{l}\right) \\
& \left(\left(G_{g^{\prime}}^{\prime}\right)\right)_{m}=\prod_{i=1}^{g \prime} \prod_{k=0}^{m-1}\left(G_{b^{\prime}}^{\prime k}\right)=\prod_{k=0}^{m-1} \prod_{i=1}^{g \prime}\left(G_{g^{\prime}}^{\prime k}\right) \\
& \left(\left(H_{h^{\prime}}^{\prime}\right)\right)_{m}=\prod_{i=1}^{h \prime} \prod_{k=0}^{m-1}\left(H_{i}^{\prime k}\right)=\prod_{k=0}^{m-1} \prod_{i=1}^{h \prime}\left(H_{i}^{\prime k}\right)
\end{aligned}
$$

write the following term as:

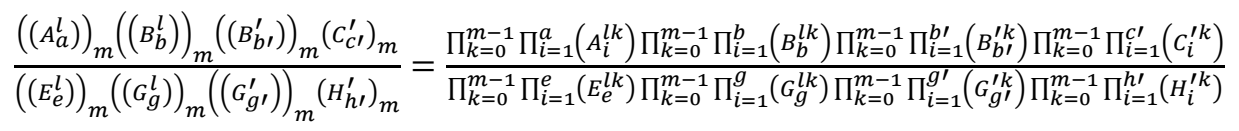

Which can be further simplified as follows:

$$
\frac{\left(\left(A_{a}^{l}\right)\right)_{m}\left(\left(B_{b}^{l}\right)\right)_{m}\left(\left(B_{b^{\prime}}^{\prime}\right)\right)_{m}\left(C_{c^{\prime}}^{\prime}\right)_{m}}{\left(\left(E_{e}^{l}\right)\right)_{m}\left(\left(G_{g}^{l}\right)\right)_{m}\left(\left(G_{g \prime}^{\prime}\right)\right)_{m}\left(H_{h^{\prime}}^{\prime}\right)_{m}}=\prod_{k=0}^{m-1} \frac{\prod_{i=1}^{a}\left(A_{i}^{l k}\right)}{\prod_{i=1}^{e}\left(E_{e}^{l k}\right)} \frac{\prod_{i=1}^{b}\left(B_{b}^{l k}\right)}{\prod_{i=1}^{g}\left(G_{g}^{l k}\right)} \frac{\prod_{i=1}^{b^{\prime}}\left(B_{b^{\prime}}^{\prime k}\right)}{\prod_{i=1}^{g^{\prime}}\left(G_{g^{\prime}}^{\prime \prime}\right)} \frac{\prod_{i=1}^{c^{\prime}}\left(C_{i}^{\prime k}\right)}{\prod_{i=1}^{h \prime}\left(H_{i}^{\prime k}\right)}
$$

If we define $q_{k}$ as below

$$
q_{k}=\frac{\prod_{i=1}^{a}\left(A_{i}^{l k}\right)}{\prod_{i=1}^{e}\left(E_{e}^{l k}\right)} \frac{\prod_{i=1}^{b}\left(B_{b}^{l k}\right)}{\prod_{i=1}^{g}\left(G_{g}^{l k}\right)} \frac{\prod_{i=1}^{b \prime}\left(B_{b \prime}^{\prime k}\right)}{\prod_{i=1}^{g \prime}\left(G_{g^{\prime}}^{\prime k}\right)} \frac{\prod_{i=1}^{c \prime}\left(\left(_{i}^{\prime k}\right)\right.}{\prod_{i=1}^{h \prime}\left(H_{i}^{\prime k}\right)}
$$

Similarly, we can write

$$
p_{k}=\frac{\prod_{i=1}^{a}\left(A_{i}^{k}\right)}{\prod_{i=1}^{e}\left(E_{e}^{k}\right)} \frac{\prod_{i=1}^{b}\left(B_{b}^{k}\right)}{\prod_{i=1}^{g}\left(G_{g}^{k}\right)} \frac{\prod_{i=1}^{b}\left(B_{b \prime \prime}^{\prime \prime}\right)}{\prod_{i=1}^{g}\left(G_{g \prime \prime}^{\prime \prime}\right)} \frac{\prod_{i=1}^{c}\left(C_{i}^{k}\right)}{\prod_{i=1}^{h}\left(H_{i}^{k}\right)}
$$

Then we can eliminate the singularity in the computation of the Srivastava's triple hypergeometric series $F^{(3)}[x, y, z][18]-$ [23] as follows

$$
\frac{\left(\left(A_{a}^{l}\right)\right)_{m}\left(\left(B_{b}^{l}\right)\right)_{m}\left(\left(B_{b \prime}^{\prime}\right)\right)_{m}\left(C_{c^{\prime}}^{\prime}\right)_{m}}{\left(\left(E_{e}^{l}\right)\right)_{m}\left(\left(G_{g}^{l}\right)\right)_{m}\left(\left(G_{g^{\prime}}^{\prime}\right)\right)_{m}\left(H_{h^{\prime}}^{\prime}\right)_{m}}=\prod_{k=0}^{m-1} q_{k}
$$

$$
\frac{\left(\left(A_{a}\right)\right)_{l}\left(\left(B_{b}\right)\right)_{l}\left(\left(B_{b^{\prime \prime}}^{\prime \prime}\right)\right)_{l}\left(\left(C_{c}\right)\right)_{l}}{\left(\left(E_{e}\right)\right)_{l}\left(\left(G_{g}\right)\right)_{l}\left(\left(G_{g \prime \prime}^{\prime \prime}\right)\right)_{l}\left(\left(H_{h}\right)\right)_{l}}=\prod_{k=0}^{l-1} p_{k}
$$

Next, by defining

$$
\begin{aligned}
& Q_{m}=\prod_{k=0}^{m-1} q_{k} ; \\
& Q_{m}=\prod_{k=0}^{m-1} q_{k} ; \tilde{Q}_{m}(y)=\prod_{k=1}^{m} \frac{q_{k-1} y}{k} \\
& P_{l}=\prod_{k=0}^{l-1} p_{k} ; \\
& P_{l}=\prod_{k=0}^{l-1} p_{k} ; \tilde{P}_{l}(x)=\prod_{k=1}^{l} \frac{p_{k-1} x}{k}
\end{aligned}
$$

By substituting (244) and (246) into (226), yields 


$$
F^{(3)}[x, y, z]=\sum_{l, m \geq 0}^{\infty} \tilde{P}_{l}(x) \tilde{Q}_{m}(y)_{\tilde{a}} F_{\tilde{b}}\left[\begin{array}{l}
\left(A^{l m}\right)_{a}\left(B^{\prime m}\right)_{b \prime}\left(B^{\prime \prime l}\right)_{b^{\prime \prime}}\left(C^{\prime \prime}\right)_{c^{\prime \prime}}{ }^{\prime \prime} \\
\left(E^{l m}\right)_{e}\left(G^{\prime m}\right)_{g^{\prime}}\left(G^{\prime \prime l}\right)_{g^{\prime \prime}}\left(H^{\prime \prime}\right)_{h \prime \prime}
\end{array}\right]
$$

Finally, let us try to re-compute (247) or (225) using the technique that we just demonstrated in (223).

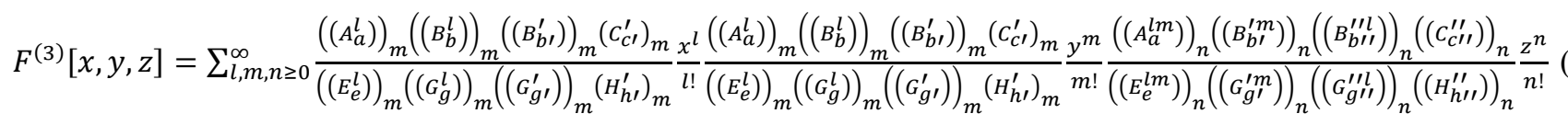

This concludes the super-efficient computation of the Srivastava's triple hypergeometric series $F^{(3)}[x, y, z][18]-[23]$ function. It is numerically very stable, computationally very efficient, and extremely accurate. I have performed an efficient

i Dr. Progri has implemented the Kampé de Fériet function (or double hypergeometric series [1]-[16]) in MATLAB in in like manner as the generalized hypergeometric series function MATLAB implementation hypergeom $(a, b, z)$ [30]. This function in called $k=k a m d e f e r(a, b, z, o p)$; where $a$ and $b$ are 3element cell vectors each, $z$ is either a vector/matrix of two rows, op is an optional vector of two elements. In the current implementation op(1) can only contain four values, 1:4 corresponding to four implementation options of the Kampé de Fériet function; op(2) is the maximum number of terms in each summation. If the user calls $k=\operatorname{kamdefer}(a, b, z)$ then $o p(1)=4$, and $o p(2)=30$. The MATLAB function kamdefer $(a, b, z)$ is proprietary information of Giftet Inc. and it is copyrighted by Giftet Inc. 2018.

ii Dr. Progri has implemented the Srivastava's triple hypergeometric series function [18]-[22] in MATLAB in in like manner as the generalized hypergeometric series function MATLAB implementation hypergeom $(a, b, z)$ [30]. This function in called $s=\operatorname{srivastava}(a, b, z, o p)$; where $a$ and $b$ are 7 element cell vectors each, $z$ is either a vector/matrix of two rows, op is an optional vector of two elements. In the current implementation op(1) can only contain four values, 1:4 corresponding to three implementation options of the Srivastava's function; op(2) is the maximum number of terms in each summation. If the user calls $s=\operatorname{srivastava}(a, b, z)$ then $o p(1)=4$, and $o p(2)=30$. The MATLAB function srivastava $(a, b, z)$ is proprietary information of Giftet Inc. and it is copyrighted by Giftet Inc. 2019.

iii Although $K_{p}(|x|)=K_{-p}(|x|)$ for $p \leq-1 / 2$; however, in a future publication we will show the required modifications of the Generalized Bessel distribution form as given by Progri 2016 [1] and 2018 [2] to produce a valid pdf and cdf. The main reason for this is that the material of this journal paper is challenging enough as is. These set of journal papers will set the stage for this future journal paper.

iv In implementation we will use Progri implementation of the hypergeometric function phypergeom(a,b,z,op), as it is as shown in this journal paper, about twenty-five times faster than the MATLAB hypergeom $(a, b, z)$. This function is proprietary information of Giftet Inc and it is part of Giftet Inc. Indoor Geolocation Systems-Theory and Applications [17] library.

$\checkmark$ This result cannot be obtained from (62) by setting $p=0$.

vi Ditto iV.

vii The function $\mathcal{F}_{21}(x ; a, d, 0)$ can also be implemented via a recursive algorithm. Substituting (109) into the first summation of (107) yields,

$$
\mathcal{F}_{21}(x ; a, d, 0)=\frac{\psi(1) \frac{\gamma(1, d x)}{d}+\sum_{k=1}^{\infty} \frac{\psi(k+1) \frac{\gamma(2 k+1, d x)}{d^{2 k+1}}}{2^{2 k}(k !)^{2}}}{C_{2}(0, d)}
$$

Where

$$
\begin{aligned}
& \gamma(1, d x)=d x e^{-d x}\left[\Phi(1,2 ; d x) \equiv \sum_{n=0}^{\infty} \frac{(1)_{n}}{(2)_{n}} \frac{(d x)^{n}}{n !}\right] \\
& \gamma(2 k+1, d x)=\frac{x^{2 k+1} d^{2 k+1} e^{-d x}}{2 k+1} \Phi(1,2+2 k ; d x)
\end{aligned}
$$

Employing the expansion for $\gamma(1, d x)$, and $\gamma(2 k+1, d x)$ we have,

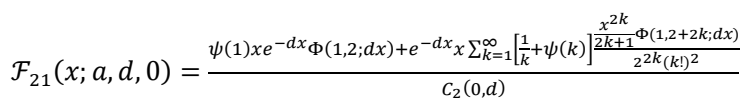

implementation of the Srivastava's triple hypergeometric series $F^{(3)}[x, y, z][18]-[23]$ function in MATLAB in like manner as the generalized hypergeometric series function hypergeom $(a, b, z)[30]$.

Equation (3) can be computed recursively, $\forall k=1,2, \cdots$

$$
\begin{aligned}
& \gamma(2 k+1, d x)=2 k \gamma(2 k, d x)-(d x)^{2 k} e^{-d x} \\
& \frac{x \Phi(1,2+2 k ; d x)}{(1+2 k)}=\mid \begin{array}{cc}
\frac{x}{1+2 k} & d=0 \\
\frac{\Phi(1,1+2 k ; d x)-1}{d} & d \neq 0
\end{array}
\end{aligned}
$$

Substituting (2) and (6) into (1) produces the final expression of $\mathcal{F}_{21}(x ; a, d, 0)$

$$
\mathcal{F}_{21}(x ; a, d, 0)=\frac{e^{-d x}\left\{\psi(1) x \Phi(1,2 ; d x)+\sum_{k=1}^{\infty}\left[\frac{1}{k}+\psi(k)\right] \frac{x_{1}^{k}}{(1)}\left[\frac{x \Phi(1,2+2 k ; d x)}{(1+2 k)}\right]\right\}}{C_{2}(0, d)}
$$

This recursive approach is fast; but, it is not nearly as accurate as the expansion by means of generalized Kampé de Fériet function.

viii There are a few differences between the generalized Kampé de Fériet function and the normal Kampé de Fériet function [11]-[13]. In the generalized Kampé de Fériet function the outer sum terminates and the Pochhammer exponent may be negative vs. in the normal Kampé de Fériet function [11]-[13] the outer sum goes to infinity and the Pochhammer exponent is always positive. More discussion about the similarities and differences of the will be published in a separate journal paper.

ix Option 1 fails after 50 terms; hence, option 1 is numerically unstable!

$x$ Option 1 fails after 50 terms; hence, option 1 is numerically unstable!

xi Warning: Infinite or Not-a-Number value encountered at $=0$.

xii Warning: Reached the limit on the maximum number of intervals in use. Approximate bound on error is 3.9e-06. The integral may not exist, or it may be difficult to approximate numerically to the requested accuracy.

Warning: Infinite or Not-a-Number value encountered.

xiii Ditto xi.

xiv Ditto xii.

$x v$ Exactly the same result can be obtained if (159) was used with Option 3 of the Kampé de Fériet function.

xvi Ditto xi.

xvii Ditto xii.

xviii There are a few differences between the generalized Kampé de Fériet function and the normal Kampé de Fériet function [11]-[13]. In the generalized Kampé de Fériet function the outer sum terminates and the Pochhammer exponent may be negative vs. in the normal Kampé de Fériet function [11]-[13] the outer sum goes to infinity and the Pochhammer exponent is always positive. More discussion about the similarities and differences of the will be published in a separate journal paper.

xix We employ the shorthand notation $p_{2 k} \equiv p+k$.

$x \mathrm{I}$ I do not believe that I have seen this symbol (or notation rather) introduced anywhere else; hence, this symbol (or notation rather) that denotes the product of three generalized Pochhammer symbols is introduced here for the first time. 